\title{
Propriedades recursivas em sistemas semidinâmicos impulsivos
}

\author{
Manuel Francisco Zuloeta Jimenez
}


SERVIÇO DE PÓS-GRADUAÇÃO DO ICMC-USP

Data de Depósito:

Assinatura:

\title{
Propriedades recursivas em sistemas semidinâmicos impulsivos
}

\author{
Manuel Francisco Zuloeta Jimenez
}

Orientador: Prof. Dr. Everaldo de Mello Bonotto

Tese apresentada ao Instituto de Ciências Matemáticas e de Computação - ICMC-USP, como parte dos requisitos para obtenção do título de Doutor em Ciências - Matemática . VERSÃO REVISADA 
Ficha catalográfica elaborada pela Biblioteca Prof. Achille Bassi e Seção Técnica de Informática, ICMC/USP com os dados fornecidos pelo(a) autor(a)

Jimenez, Manuel Francisco Zuloeta
Propriedades recursivas em sistemas semidinâmicos
impulsivos / Manuel Francisco Zuloeta Jimenez;
orientador Everaldo de Mello Bonotto. -- São
Carlos, 2014.
95 p.
Tese (Doutorado - Programa de Pós-Graduação em
Matemática) -- Instituto de Ciências Matemáticas e
de Computação, Universidade de São Paulo, 2014.
1. sistemas semidinâmicos impulsivos. 2.
propriedades recursivas. 3. estabilidade. 4.
atratores. 5. semisoluções nagativas. I. Bonotto,
Everaldo de Mello, orient. II. Título.


Arrisque-se! Toda vida é um risco. O homem que vai mais longe é geralmente aquele que está disposto a fazer e a ousar. O barco da segurança nunca vai muito além da margem.

Dale Carnegie 



\section{Agradecimentos}

Tentarei expressar em poucas linhas minha gratidão às pessoas que de alguma forma estiveram presentes e que muito me ajudaram a escrever as páginas de mais uma vitória.

Inicialmente gostaria de expressar minha eterna gratidão a Deus pelo amor, pela força que me impulsiona a lutar pelos meus objetivos e por me ter permitido, sempre com muita fé, ultrapassar todos os obstáculos.

Agradeço à minha família que sempre esteve presente para me dar suporte de forma incondicional em todos os aspectos possíveis durante meus estudos, mesmo distante. Aos meus pais, Juan e Gladis, pela dedicação, pela educação e pelo esforço para comigo e meus irmãos. Aos meus irmãos, Fernando, Elvis, Angel, Jose, Clara e Paola, pelo incentivo, carinho e compreensão ao longo destes anos de dedicação à Matemática. Aos meus sobrinhos, Crisby, Diego, Carmen, Dayana, Josue e Maricielo, obrigado por fazer de minha vida mais feliz. Amo todos vocês!

Agradeço ao meu orientador, Prof. Everaldo de Mello Bonotto, pelo apoio, pela amizade, pela dedicação e por estar sempre disposto em ajudar no que fosse preciso. Pelo constante incentivo, por acreditar sempre no meu trabalho, por me ajudar a trilhar este caminho difícil, mas que no final, é compensador. Agradeço também à Profa. Suzete Afonso ...muito obrigado!

Agradeço aos professores do departamento de Matemática do ICMC, pelos ensinamentos, apoio, motivação, pela amizade e pelas aulas ministradas durante este período com as quais muito aprendi; todos são modelos de pessoas e profissionais que eu vou ter para o resto da minha vida.

Agradeço aos colegas e amigos que fiz nessa jornada, Nancy, José Bravo, Luis Espinoza, Telma, Luis Enrique, Gian, Norbil, Lizandro, Irma, Vanessa, Alessandra, Lourdes, Eber, Christian, Jaqueline, Rodolfo, Ginara, Matheus, Jose Valencia, Patricia e muitos outros... Só tenho a agradecer por 
ter encontrado cada um de vocês! Obrigado pelo exemplo de pessoas íntegras que são, gigantes que me emprestam seu ombro para apoiar e ver mais longe. Obrigado pelas longas conversas, pelas horas de estudo, às horas de lazer, pelos churrasquinhos, pelos dias de futebol e por todos os momentos inesquecíveis vividos.

Não poderia deixar de agradecer aos funcionários do ICMC por estarem sempre à disposição e tornarem nossa vivência mais agradável

Agradeço à CAPES e FAPESP pelo suporte financeiro à este trabalho, sem este apoio nada disso seria possível.

Enfim, se for agradecer a cada um, não acabaria nunca, pois são tantas as pessoas especiais que passaram e marcaram esta jornada...

Mais uma vez a todos vocês muito obrigado!!! 


\section{Resumo}

A teoria de sistemas semidinâmicos impulsivos é um capítulo importante e moderno da teoria de sistemas dinâmicos topológicos. Sistemas impulsivos descrevem processos de evolução que sofrem variações de estado de curta duração e que podem ser consideradas instantâneas. Os sistemas impulsivos admitem vários fenômenos interessantes às vezes, por causa da sua "irregularidade", e às vezes por causa da sua "regularidade". Para muitos fenômenos naturais, os modelos determinísticos mais realistas são frequentemente descritos por sistemas que envolvem impulsos. Esta teoria vem sendo desenvolvida continuamente.

O presente trabalho apresenta resultados originais sobre a teoria de conjuntos minimais, movimentos recorrentes, movimentos quase periódicos e fracamente quase periódicos, teoria de estabilidade de Lyapunov, teoria da quase estabilidade de Zhukovskij e, finalmente, a construção de trajetórias negativas para sistemas semidinâmicos com impulsos.

Os resultados novos apresentados neste trabalho estão contidos em três artigos, dos quais dois já foram aceitos para publicação. Veja [13], [14] e [15]. 



\section{Abstract}

The theory of impulsive semidynamical systems is an important and modern chapter of the theory of topological dynamical systems. Impulsive systems describe the evolution of process whose continuous dynamics are interrupted by abrupt changes of state. This kind of systems admits various interesting phenomena sometimes, because of their "irregularity", and sometimes because of their "regularity". In many natural phenomena, the real deterministic models are often described by systems which involve impulses. This theory has been developed continuously.

This work presents original results involving the theory of minimal sets, recurrent motions, almost periodic and weakly almost periodic motions, the study of Lyapunov stability and Zhukovshij Quasi stability and the construction of negative trajectories for impulsive semidynamical systems.

The new results presented in this work are contained in three papers namely [13], [14] and [15]. 



\section{Sumário}

$\begin{array}{ll}\text { Introdução } & 1\end{array}$

$\begin{array}{ll}\text { Notações } & 5\end{array}$

1 Sistemas semidinâmicos impulsivos $\quad 7$

1.1 Descrição de um sistema semidinâmico com impulsos . . . . . . . . . . . . . . . 7

1.2 Continuidade da função $\phi \ldots \ldots \ldots \ldots$. . . . . . . . . . . . . . . . . . . . . .

1.3 Invariância . . . . . . . . . . . . . . . . . . . . 17

1.4 Conjuntos limite . . . . . . . . . . . . . . . . . . . . . . . . . 19

1.5 Hipóteses gerais . . . . . . . . . . . . . . . . . . . . . . 24

2 Conjuntos minimais $\quad 25$

2.1 Conjuntos minimais . . . . . . . . . . . . . . . . 25

3 Movimentos recorrentes e quase periódicos 35

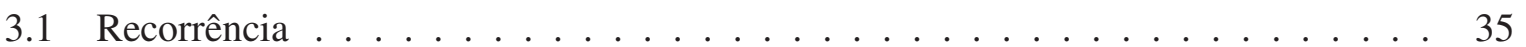

3.2 Movimentos quase periódicos . . . . . . . . . . . . . 46

4 Estabilidade de Lyapunov e de Zhukovskij 
4.1 Estabilidade de Lyapunov . . . . . . . . . . . . . . . . . . . . 51 51

4.2 Quase estabilidade de Zhukovskij . . . . . . . . . . . . . . . 63

5 Trajetórias negativas em sistemas semidinâmicos impulsivos $\quad 69$

5.1 Trajetória negativa impulsiva . . . . . . . . . . . . . . . . . . . 69

5.2 Convergência para semisoluções negativas impulsivas . . . . . . . . . . . . 76

5.3 Invariância e conjuntos limite . . . . . . . . . . . . . . . 80

5.4 Conjuntos fracamente minimais $\ldots \ldots \ldots \ldots$

$\begin{array}{ll}\text { Referências Bibliográficas } & 93\end{array}$ 


\section{Introdução}

A teoria de equações diferenciais impulsivas (EDIs) estuda o comportamento de processos de evolução que sofrem variações de estado de curta duração e que podem ser consideradas instantâneas. Tais equações constituem em exemplos de sistemas dinâmicos de dimensão infinita, apresentando dinâmica complexa. A teoria básica de EDIs pode ser encontrada em [24].

As aplicações das EDIs ocorrem, especialmente, nas áreas de farmacocinética, finanças, tecnologia química, medicina, áreas de controle, engenharia, física, biologia, entre outras. Esta teoria é uma importante área de investigação que aparece naturalmente na descrição de processos de evolução de vários problemas da realidade.

Uma das aplicações da teoria das EDIs é a teoria de sistemas dinâmicos com impulsos. Sistemas dinâmicos impulsivos é um capítulo importante e moderno da teoria de sistemas dinâmicos topológicos. Tais sistemas admitem vários fenômenos interessantes às vezes, por causa da sua "irregularidade", e às vezes por causa da sua "regularidade". Recentemente, vários artigos sobre este tópico foram publicados. Veja, por exemplo, [5, 6, 7, 8, 9, 10, 11, 12], [16, 17, 18, 19, 20, 21, 22] e [23].

Muitos dos resultados da teoria de sistemas semidinâmicos com impulsos são generalizações de resultados da teoria clássica de sistemas dinâmicos contínuos. Além disso, o estudo de sistemas impulsivos leva a novas demonstrações de resultados da teoria de sistemas dinâmicos contínuos.

Neste trabalho, dando continuidade no estudo da teoria de sistemas com impulsos, apresentamos resultados que envolvem conjuntos minimais, movimentos recorrentes, movimentos quase periódicos e fracamente quase periódicos. Estudamos, também, a teoria de estabilidade de Lyapunov e quase estabilidade de Zhukovskij para sistemas impulsivos e apresentamos a construção da teoria de trajetórias negativas para sistemas semidinâmicos com impulsos. 
Organizamos este trabalho da seguinte forma. No Capítulo 1, apresentamos os conceitos básicos da teoria de sistemas semidinâmicos com impulsos. Na Seção 1.1, apresentamos a definição de um sistema semidinâmico impulsivo, representado por $(X, \pi ; M, I)$, onde $X$ é um espaço métrico, $(X, \pi)$ é uma sistema semidinâmico contínuo, $M$ é o conjunto de impulsos e $I$ é a função impulso. A órbita positiva impulsiva de um ponto $x \in X$ é definida pelo conjunto $\tilde{\pi}^{+}(x)=\{\tilde{\pi}(x, t): t \in[0, T(x))\}$. Na Seção 1.2, estudamos a continuidade da função $\phi$ que representa o menor tempo positivo para o qual a trajetória positiva de um ponto encontra o conjunto impulsivo. A Seção 1.3 dedica-se ao estudo de conjuntos invariantes em sistemas com impulsos. Já a Seção 1.4 estuda propriedades de conjuntos limite no sistema com impulsos. Na Seção 1.5, exibimos as condições gerais que iremos assumir em todo este trabalho. As principais referências para este capítulo são [2], [3], [4], [5], [6], [7], [9], [11], [16], [17], [18], [20] e [23].

No Capítulo 2, apresentamos a teoria de conjuntos minimais. Definimos o conceito de conjuntos minimais, para sistemas impulsivos, de forma similar à definição clássica apresentada no caso de sistemas contínuos. Mostramos que nossa definição de minimalidade é equivalente a definição dada pelo matemático Kaul em [21], veja Teorema 2.1.3. O Teorema 2.1.4 mostra que um conjunto $A \subset X$ é minimal em $(X, \pi ; M, I)$ se, e somente se, $A$ for igual ao conjunto limite positivo de qualquer ponto que pertença ao conjunto $A \backslash M$. No Teorema 2.1.11, mostramos condições suficientes para que um conjunto compacto e não vazio contenha um subconjunto minimal em $(X, \pi ; M, I)$. Os resultados desse capítulo estão em [13].

O Capítulo 3 estuda a teoria de movimentos recorrentes e quase periódicos. Na Seção 3.1, definimos o conceito de recorrência para sistemas semidinâmicos com impulsos e estudamos algumas propriedades topológicas de movimentos recorrentes. No Teorema 3.1.3, mostramos que se $A \subset X$ é um subconjunto compacto e minimal, então qualquer ponto $x \in A \backslash M$ é recorrente em $(X, \pi ; M, I)$. O Teorema 3.1.4 mostra que se $X$ é completo e $\tilde{\pi}^{+}(x)$ é recorrente em $(X, \pi ; M, I)$, então o fecho $\overline{\widetilde{\pi}^{+}(x)}$ é um conjunto compacto. Além disso, se $x \notin M$ então $\overline{\widetilde{\pi}^{+}(x)}$ é minimal.

Apresentamos, também, o conceito de conjunto relativamente denso, e mostramos no Teorema 3.1.6 que se $(X, \pi ; M, I)$ é um sistema semidinâmico impulsivo tal que $\overline{\widetilde{\pi}^{+}(x)}$ é compacto para algum $x \in X \backslash M$, então a órbita positiva $\tilde{\pi}^{+}(x)$ é recorrente se, e somente se, para cada $\varepsilon>0$ o conjunto $K_{\varepsilon}=\left\{t \in \mathbb{R}_{+}: d(x, \tilde{\pi}(x, t))<\varepsilon\right\}$ é relativamente denso. 
Na Seção 3.2, definimos o conceito de movimentos quase periódicos. Estabelecemos condições para que um ponto quase periódico seja recorrente em um sistema impulsivo, veja Teorema 3.2.3. Finalizamos o capítulo com resultados que envolvem conceitos de periodicidade e minimalidade, veja os Teoremas 3.2.6 e 3.2.7 e o Corolário 3.2.8. Os resultados novos deste capítulo estão presentes no artigo [13].

No Capítulo 4, apresentamos a teoria de estabilidade de Lyapunov e a teoria de quase estabilidade de Zhukovskij. Na Seção 4.1, definimos o conceito de movimento fracamente quase $\tilde{\pi}$-periódico e estabelecemos condições para que um ponto fracamente quase $\tilde{\pi}$-periódico seja $\tilde{\pi}$-recorrente, veja o Teorema 4.1.2.

No Teorema 4.1.3, mostramos que se $x \in X \backslash M$ e $x$ é quase periódico em $(X, \pi ; M, I)$ então $x \in \widetilde{L}^{+}(x)$. Mostramos, também, que a recíproca desse resultado não é válida em geral, veja Exemplo 4.1.5. Entretanto, supondo que $x$ seja recorrente e Lyapunov estável em $(X, \pi ; M, I)$ então encontramos condições suficientes para que $x$ seja fracamente quase periódico, veja Teorema 4.1.7. Outro resultado importante, mostra que, o conjunto limite positivo de um ponto $x \in X$ é minimal se, e somente se, a órbita positiva de $x$ aproxima-se uniformemente desse conjunto limite, veja Teorema 4.1.11. Na Seção 4.2, estudamos a teoria de quase estabilidade de Zhukovskij para sistemas impulsivos. Este tipo de estabilidade foi inicialmente introduzido por Changming Ding em [19]. Sob condição de quase estabilidade de Zhukovskij, mostramos que um conjunto limite é minimal, veja Teorema 4.2.7. Obtemos, também, alguns resultados sobre atratores uniformes, veja Lema 4.2.14, Proposição 4.2.15 e Teorema 4.2.16.

Finalmente, no Capítulo 5, apresentamos a noção de semisolução negativa para sistemas semidinâmicos impulsivos. Este capítulo está dividido em quatro seções. Na Seção 5.1, construímos a teoria de semisoluções negativas para sistemas impulsivos. Na Seção 5.2, definimos uma função que representa o maior tempo negativo para o qual a semisolução negativa contínua de um ponto $x$ encontra o conjunto $I(M)$. Através dessa função, estabelecemos um teorema de convergência para semisoluções negativas em sistemas com impulsos, veja Teorema 5.2.3.

Na Seção 5.3, definimos os conceitos de conjuntos negativamente fracamente invariantes, negativamente fortemente invariantes e invariantes. Estabelecemos os conceitos de conjunto limite negativo e do prolongamento do conjunto limite negativo. Resultados de invariância positiva e ne- 
gativa estão presentes nos Teoremas 5.3.5, 5.3.6, 5.3.7 e 5.3.11. Finalmente, na Seção 5.4, definimos o conceito de conjuntos fortemente (fracamente) minimais e mostramos algumas propriedades sobre conjuntos fracamente minimais e negativamente fortemente invariantes, veja Teorema 5.4.4. 


\section{Notações}

Seguem algumas notações que aparecem no contexto deste trabalho.

Sejam $(X, d)$ um espaço métrico com métrica $d, x \in X, \varepsilon>0, n$ um natural e $A$ um subconjunto não vazio de $X$, isto é, $A \subset X$ e $A \neq \emptyset$.

- $\mathbb{R}$ representa o conjunto dos números reais;

- $\mathbb{R}_{+}$representa o conjunto dos números reais não negativos;

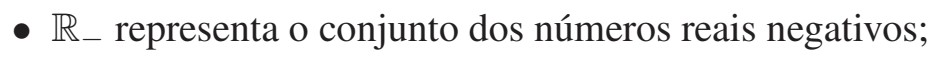

- $\mathbb{N}$ representa o conjunto dos números naturais, $\mathbb{N}=\{0,1,2, \ldots\}$;

- $\mathbb{N}^{*}$ representa o conjunto dos números naturais estritamente positivos, ou seja, $\mathbb{N}^{*}=\mathbb{N} \backslash\{0\}$;

- $\mathbb{R}^{n}$ representa o espaço euclidiano $n$-dimensional;

- $\bar{A}$ representa o fecho de $A$ em $X$;

- $\partial A$ representa a fronteira de $A$ em $X$;

- $d(x, A)=\inf \{d(x, y): y \in A\}$

- $B(x, \varepsilon)=\{y \in X: d(y, x)<\varepsilon\}$;

- $B(A, \varepsilon)=\{x \in X: d(x, A)<\varepsilon\}$.

Vamos representar por $\left\{x_{n}\right\}_{n \geq 1}$ uma seqüência em $X$, onde $n$ pertence ao conjunto dos números naturais. Às vezes, representaremos $\lim _{n \rightarrow+\infty} x_{n}=x$ simplesmente como

$$
x_{n} \stackrel{n \rightarrow+\infty}{\longrightarrow} x
$$


Quando um conceito é introduzido pela primeira vez, as palavras que o definem estão em negrito. Indicamos o final de uma demonstração com o símbolo $\square$.

Em cada resultado deste trabalho apresentamos uma referência bibliográfica. Os resultados dos trabalhos do autor estão em negrito. 


\section{Capítulo}

\section{Sistemas semidinâmicos impulsivos}

Neste capítulo, apresentamos a teoria elementar de sistemas semidinâmicos impulsivos. As referências utilizadas foram [2], [3], [4], [5], [6], [7], [9], [11], [16], [17], [18] , [20] e [23].

\subsection{Descrição de um sistema semidinâmico com impulsos}

Seja $(X, d)$ um espaço métrico. A tripla $\left(X, \pi, \mathbb{R}_{+}\right)$é chamada de sistema semidinâmico em $X$ ou $\pi$ é um sistema semidinâmico em $X$, se a aplicação $\pi: X \times \mathbb{R}_{+} \rightarrow X$ satisfaz as seguintes propriedades:

i) $\pi(x, 0)=x$ para todo $x \in X$;

ii) $\pi(\pi(x, t), s)=\pi(x, t+s)$, quaisquer que sejam $x \in X$ e $t, s \in \mathbb{R}_{+}$;

iii) $\pi$ é contínua em $X \times \mathbb{R}_{+}$.

Vamos denotar um sistema semidinâmico simplesmente por $(X, \pi)$. Para cada $x \in X$, consideramos a função contínua $\pi_{x}: \mathbb{R}_{+} \rightarrow X$ dada por $\pi_{x}(t)=\pi(x, t)$ e chamamos esta função de movimento de $x$.

A órbita positiva de $x$ no sistema $(X, \pi)$ é definida pelo conjunto $\pi^{+}(x)=\left\{\pi(x, t): t \in \mathbb{R}_{+}\right\}$. Dados $A \subset X$ e $t \geq 0$, definimos

$$
\pi^{+}(A)=\bigcup_{x \in A} \pi^{+}(x) \quad \text { e } \quad \pi(A, t)=\bigcup_{x \in A} \pi(x, t) .
$$


Dizemos que um conjunto $A \subset X$ é positivamente $\pi$-invariante se $\pi^{+}(A) \subset A$.

Dados $t \geq 0$ e $x \in X$, definimos o conjunto $F(x, t)$ da seguinte maneira

$$
F(x, t)=\{y \in X: \pi(y, t)=x\}
$$

e, para $\Delta \subset[0,+\infty)$ e $D \subset X$, definimos $F(D, \Delta)=\cup\{F(x, t): x \in D$ e $t \in \Delta\}$.

Definição 1.1.1 Um ponto $x \in X$ é chamado de ponto inicial em $(X, \pi)$ se $F(x, t)=\emptyset$ para todo $t>0$.

A teoria de sistemas dinâmicos e semidinâmicos contínuos pode ser encontrada em [2] e [3].

No que segue, definimos o conceito de sistemas semidinâmicos sob ação de impulsos.

Definição 1.1.2 Um sistema semidinâmico impulsivo em $X$, denotado por $(X, \pi ; M, I)$, consiste de um sistema semidinâmico $(X, \pi)$ juntamente com um subconjunto fechado não vazio $M \subset X$ satisfazendo a condição de que para cada $x \in M$, existe um $\varepsilon_{x}>0$ tal que

$$
F\left(x,\left(0, \varepsilon_{x}\right)\right) \cap M=\emptyset \quad \text { e } \quad \pi\left(x,\left(0, \varepsilon_{x}\right)\right) \cap M=\emptyset
$$

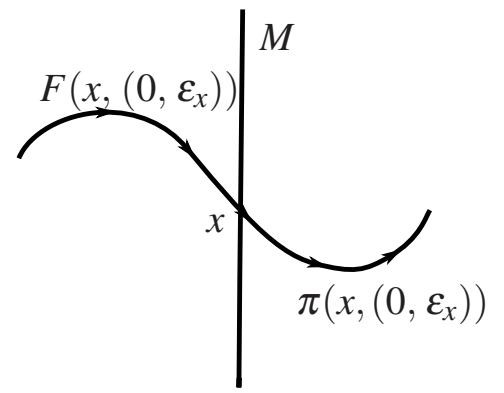

Figura 1.1: Trajetória através de $x$.

e uma função contínua $I: M \rightarrow X$ responsável pelas descontinuidades do sistema impulsivo. Chamamos o conjunto $M$ de conjunto impulsivo e a função $I$ de função impulso. Na sequência, vamos descrever como a função $I$ atua nas trajetórias do sistema impulsivo.

O lema a seguir apresenta condições para obtermos a existência do menor tempo estritamente positivo para o qual a trajetória de um sistema impulsivo encontra o conjunto impulsivo $M$. 
Lema 1.1.3 [5, Lema 2.1] Seja $(X, \pi ; M, I)$ um sistema semidinâmico impulsivo. Então, para qualquer $x \in X$ tal que $\pi(x,(0,+\infty)) \cap M \neq \emptyset$, existe um número real positivo $s_{1}>0$, de maneira que $\pi(x, t) \notin M$ para $0<t<s_{1}$ e $\pi\left(x, s_{1}\right) \in M$.

Demonstração: Como $\pi(x,(0,+\infty)) \cap M \neq \emptyset$, existe um número $t_{1}>0$ tal que $\pi\left(x, t_{1}\right) \in M$. Como $\pi_{x}: \mathbb{R}_{+} \longrightarrow X$ é contínua e $M$ é fechado e não vazio, então o subconjunto compacto $\left[0, t_{1}\right] \cap \pi_{x}^{-1}(M)$ de $\mathbb{R}_{+}$possui um menor elemento, digamos $s_{1}$, o qual satisfaz o lema.

Nas definições que seguem, $(X, \pi ; M, I)$ denota um sistema semidinâmico impulsivo.

Definição 1.1.4 Definimos a função $\phi: X \rightarrow(0,+\infty]$ pela lei

$$
\phi(x)= \begin{cases}s, & \text { se } \pi(x, s) \in M \text { e } \pi(x, t) \notin M \text { para } 0<t<s \\ +\infty, & \text { se } M^{+}(x)=\emptyset\end{cases}
$$

onde $M^{+}(x)=\pi(x,(0,+\infty)) \cap M, x \in X$. Se $M^{+}(x) \neq \emptyset$, segue que o valor $\phi(x)$ representa o menor tempo estritamente positivo para o qual a trajetória de $x$ encontra $M$.

Definição 1.1.5 Dado $x \in X$, chamamos $\pi(x, \phi(x))$ de ponto impulsivo de $x$.

Definição 1.1.6 A trajetória impulsiva de $x$ em $(X, \pi ; M, I)$ é uma aplicação $\widetilde{\pi}_{x}$ definida em um intervalo $I_{x} \subset \mathbb{R}_{+}$com valores em $X$ dada indutivamente da seguinte forma: Se $M^{+}(x)=\emptyset$, então $\tilde{\pi}_{x}(t)=\pi(x, t)$ para todo $t \in \mathbb{R}_{+}$e $\phi(x)=+\infty$. Porém se $M^{+}(x) \neq \emptyset$, segue do Lema 1.1.3 que existe um menor positivo $s_{0} \in \mathbb{R}_{+}$de maneira que $\pi\left(x, s_{0}\right)=x_{1} \in M$ e $\pi(x, t) \notin M$, para $0<t<s_{0}$. Então definimos $\tilde{\pi}_{x}$ sobre $\left[0, s_{0}\right]$ como sendo

$$
\tilde{\pi}_{x}(t)= \begin{cases}\pi(x, t), & 0 \leq t<s_{0} \\ x_{1}^{+}, & t=s_{0}\end{cases}
$$

onde $x_{1}^{+}=I\left(x_{1}\right)$ e $\phi(x)=s_{0}$. Vamos denotar $x$ por $x_{0}^{+}$.

Como $s_{0}<+\infty$, o processo continua mas, agora, iniciando em $x_{1}^{+}$. Deste modo, caso $M^{+}\left(x_{1}^{+}\right)=\emptyset$, definimos $\tilde{\pi}_{x}(t)=\pi\left(x_{1}^{+}, t-s_{0}\right)$ para $s_{0} \leq t<+\infty$ e, neste caso, $\phi\left(x_{1}^{+}\right)=+\infty$. Agora, 
se $M^{+}\left(x_{1}^{+}\right) \neq \emptyset$, segue novamente do Lema 1.1.3, que existe um menor positivo $s_{1} \in \mathbb{R}_{+}$tal que $\pi\left(x_{1}^{+}, s_{1}\right)=x_{2} \in M$ e $\pi\left(x_{1}^{+}, t-s_{0}\right) \notin M$ para $s_{0}<t<s_{0}+s_{1}$. Definimos $\tilde{\pi}_{x}$ sobre $\left[s_{0}, s_{0}+s_{1}\right]$ por

$$
\tilde{\pi}_{x}(t)= \begin{cases}\pi\left(x_{1}^{+}, t-s_{0}\right), & s_{0} \leq t<s_{0}+s_{1}, \\ x_{2}^{+}, & t=s_{0}+s_{1},\end{cases}
$$

onde $x_{2}^{+}=I\left(x_{2}\right)$ e $\phi\left(x_{1}^{+}\right)=s_{1}$.

Suponhamos, agora, que $\widetilde{\pi}_{x}$ esteja definida no intervalo $\left[t_{n-1}, t_{n}\right]$ e que $\widetilde{\pi}_{x}\left(t_{n}\right)=x_{n}^{+}$, onde $t_{0}=0$ e $t_{n}=\sum_{i=0}^{n-1} s_{i}$ para $n=1,2,3, \ldots$ Se $M^{+}\left(x_{n}^{+}\right)=\emptyset$, então $\tilde{\pi}_{x}(t)=\pi\left(x_{n}^{+}, t-t_{n}\right)$ para $t_{n} \leq t<+\infty \mathrm{e}$ $\phi\left(x_{n}^{+}\right)=+\infty$. Se $M^{+}\left(x_{n}^{+}\right) \neq \emptyset$, então existe $s_{n} \in \mathbb{R}_{+}$tal que $\pi\left(x_{n}^{+}, s_{n}\right)=x_{n+1} \in M$ e $\pi\left(x_{n}^{+}, t-t_{n}\right) \notin$ $M$, para $t_{n}<t<t_{n+1}$. Além disso,

$$
\tilde{\pi}_{x}(t)= \begin{cases}\pi\left(x_{n}^{+}, t-t_{n}\right), & t_{n} \leq t<t_{n+1}, \\ x_{n+1}^{+}, & t=t_{n+1},\end{cases}
$$

onde $x_{n+1}^{+}=I\left(x_{n+1}\right)$ e $\phi\left(x_{n}^{+}\right)=s_{n}$.

Observemos que $\widetilde{\pi}_{x}$ está definida sobre cada $\left[t_{n}, t_{n+1}\right]$, onde $t_{n+1}=\sum_{i=0}^{n} s_{i}$, ou seja, $\widetilde{\pi}_{x}$ está definida sobre $\left[0, t_{n+1}\right]$. A Figura 1.2 apresenta a trajetória de um ponto $x \in X$ em um sistema impulsivo.

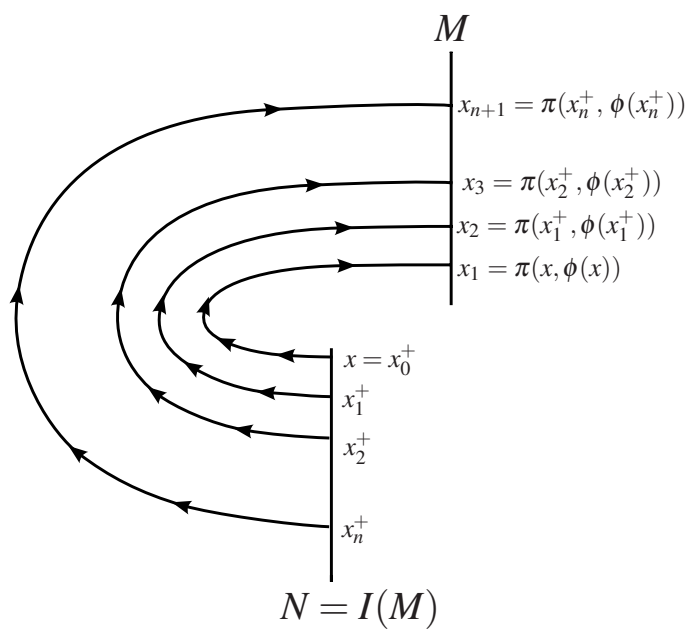

Figura 1.2: Trajetória impulsiva do ponto $x \in X$ no sistema $(X, \pi ; M, I)$.

O processo acima termina após um número finito de passos, se $M^{+}\left(x_{n}^{+}\right)=\emptyset$ para algum $n \in \mathbb{N}$, 
ou ele continua indefinidamente, se $M^{+}\left(x_{n}^{+}\right) \neq \emptyset$ para cada $n=1,2,3, \ldots$, e neste caso $\widetilde{\pi}_{x}$ está definida no intervalo $[0, T(x))$, onde $T(x)=\sum_{i=0}^{\infty} s_{i}$ e $x \in X$. Representamos a órbita positiva impulsiva de $x \in X$ pelo conjunto

$$
\tilde{\pi}^{+}(x)=\{\tilde{\pi}(x, t): t \in[0, T(x))\}
$$

Dado $A \subset X$ definimos $\tilde{\pi}^{+}(A)=\cup\left\{\tilde{\pi}^{+}(x): x \in A\right\}$.

\section{Observação 1.1.7}

i) Escrevemos $\tilde{\pi}_{x}(t)=\tilde{\pi}(x, t)$, para qualquer $t$ em $[0, T(x))$. Pela descrição do sistema impulsivo temos que para qualquer $t$ tal que $0 \leq t<T(x)$, existem $k \in\{1,2,3, \ldots\}$ e $0 \leq t^{\prime}<\phi\left(x_{k-1}^{+}\right)$ tais que $t=\sum_{i=0}^{k-1} \mu\left(x_{i-1}^{+}\right)+t^{\prime}$ e $\tilde{\pi}(x, t)=\pi\left(x_{k-1}^{+}, t^{\prime}\right)$, onde $x=x_{0}^{+}, \mu\left(x_{-1}^{+}\right)=0$ e $\mu\left(x_{i}^{+}\right)=\phi\left(x_{i}^{+}\right)$ para todo $i=0,1,2,3, \ldots$. Mesmo que a função $\phi$ é estritamente positiva, vamos escrever por conveniência a expressão $t=\sum_{i=0}^{k-1} \phi\left(x_{i-1}^{+}\right)+t^{\prime} \operatorname{com} \phi\left(x_{-1}^{+}\right)=0$.

ii) Note que, pela definição da órbita impulsiva, $\tilde{\pi}(x, t) \notin M$, para todo $t>0$.

A proposição seguinte nos diz que $\tilde{\pi}$ satisfaz o princípio da identidade e a condição de semigrupo.

Proposição 1.1.8 [6, Proposição 2.1] Seja $(X, \pi ; M, I)$ um sistema semidinâmico impulsivo. Se $x \in X$, então:

a) $\tilde{\pi}(x, 0)=x$

b) $\tilde{\pi}(\tilde{\pi}(x, t), s)=\tilde{\pi}(x, t+s)$ para todos $t, s \in[0, T(x))$ tais que $t+s \in[0, T(x))$.

Demonstração: Se $\widetilde{\pi}$ for contínua, a conclusão é imediata.

a) Dado $x \in X$, temos que $\tilde{\pi}(x, t)=\pi(x, t)$ para $0 \leq t<\phi(x)$. Então $\tilde{\pi}(x, 0)=\pi(x, 0)=x$.

b) Sejam $t, s \in[0, T(x))$ tais que $t+s \in[0, T(x))$, e consideremos $y=\tilde{\pi}(x, t)$. Pela Observação 1.1.7, podemos escrever

$$
\widetilde{\pi}(x, t)=\pi\left(x_{k-1}^{+}, t^{\prime}\right)
$$


para algum $k \geq 1$, onde $t=\sum_{j=0}^{k-1} \phi\left(x_{j-1}^{+}\right)+t^{\prime}$ e $0 \leq t^{\prime}<\phi\left(x_{k-1}^{+}\right)$(lembremos que estamos denotando $x=x_{0}^{+}$e $\left.\phi\left(x_{-1}^{+}\right)=0\right)$. De mesma forma, podemos escrever

$$
\tilde{\pi}(y, s)=\pi\left(y_{\ell-1}^{+}, s^{\prime}\right)
$$

para algum $\ell \geq 1$, onde $s=\sum_{j=0}^{\ell-1} \phi\left(y_{j-1}^{+}\right)+s^{\prime}$ e $0 \leq s^{\prime}<\phi\left(y_{\ell-1}^{+}\right)\left(\operatorname{com} y=y_{0}^{+}\right.$e $\left.\phi\left(y_{-1}^{+}\right)=0\right)$.

Se $\ell=1$, obtemos $s=s^{\prime}<\phi(y)=\phi\left(x_{k-1}^{+}\right)-t^{\prime}$ e $t+s=\sum_{j=0}^{k-1} \phi\left(x_{j-1}^{+}\right)+t^{\prime}+s^{\prime}$. Portanto

$$
\tilde{\pi}(\tilde{\pi}(x, t), s)=\tilde{\pi}(y, s)=\pi(y, s)=\pi\left(x_{k-1}^{+}, t^{\prime}+s^{\prime}\right)=\tilde{\pi}(x, t+s) .
$$

Agora se $\ell>1$, como $y=\tilde{\pi}(x, t)=\pi\left(x_{k-1}^{+}, t^{\prime}\right)$, obtemos

$$
\phi(y)=\phi\left(x_{k-1}^{+}\right)-t^{\prime}, \quad y_{j}^{+}=x_{k-1+j}^{+} \quad \text { e } \quad \phi\left(y_{j}^{+}\right)=\phi\left(x_{k-1+j}^{+}\right)
$$

para todo $j \geq 1$. Segue que

$$
\begin{aligned}
s & =\phi\left(y_{0}^{+}\right)+\phi\left(y_{1}^{+}\right)+\ldots+\phi\left(y_{\ell-2}^{+}\right)+s^{\prime} \\
& =\phi\left(x_{k-1}^{+}\right)-t^{\prime}+\phi\left(x_{k}^{+}\right)+\ldots+\phi\left(x_{k+\ell-3}^{+}\right)+s^{\prime} .
\end{aligned}
$$

Então,

$$
t+s=\left(\sum_{j=0}^{k+\ell-2} \phi\left(x_{j-1}^{+}\right)\right)+s^{\prime} \quad \text { e } \quad 0 \leq s^{\prime}<\phi\left(x_{k+\ell-2}^{+}\right) .
$$

Portanto, $\tilde{\pi}(\tilde{\pi}(x, t), s)=\tilde{\pi}(y, s)=\pi\left(y_{\ell-1}^{+}, s^{\prime}\right)=\pi\left(x_{k+\ell-2}^{+}, s^{\prime}\right)=\tilde{\pi}(x, t+s)$.

Observação 1.1.9 É importante notarmos que se $x \in M$ então $\tilde{\pi}(x, t)=\pi(x, t)$ para todo $0 \leq t<\phi(x)$, ou seja, não há impulso no instante $t=0$. Para que a trajetória de um ponto $x \in X$ sofra impulso, devemos encontrar o menor tempo estritamente positivo para o qual esta trajetória encontra $M$.

Observação 1.1.10 Vamos assumir neste trabalho que $T(x)=+\infty$ para todo $x \in X$, isto é, $\widetilde{\pi}_{x}$ está definida para todo $t \geq 0$ qualquer que seja $x \in X$. 


\subsection{Continuidade da função $\phi$}

Na Definição 1.1.4 da seção anterior, definimos a função $\phi$ que representa o menor tempo positivo para o qual a trajetória de um ponto $x \in X$ encontra o conjunto impulsivo $M$. No que segue, estudamos a continuidade da função $\phi$.

Definição 1.2.1 Seja $(X, \pi)$ um sistema semidinâmico. Um conjunto fechado $S$ contendo $x \in X$ é chamado de seção ou $\lambda$ - seção através de $x, \operatorname{com} \lambda$ real positivo, se existe um conjunto fechado $L$ tal que:

i) $F(L, \lambda)=S$;

ii) $F(L,[0,2 \lambda])$ é uma vizinhança de $x$;

iii) $F(L, \mu) \cap F(L, v)=\emptyset$, para $0 \leq \mu<v \leq 2 \lambda$.

Denominamos o conjunto $F(L,[0,2 \lambda])$ de tubo (ou $\lambda$-tubo) e o conjunto $L$ de barra.

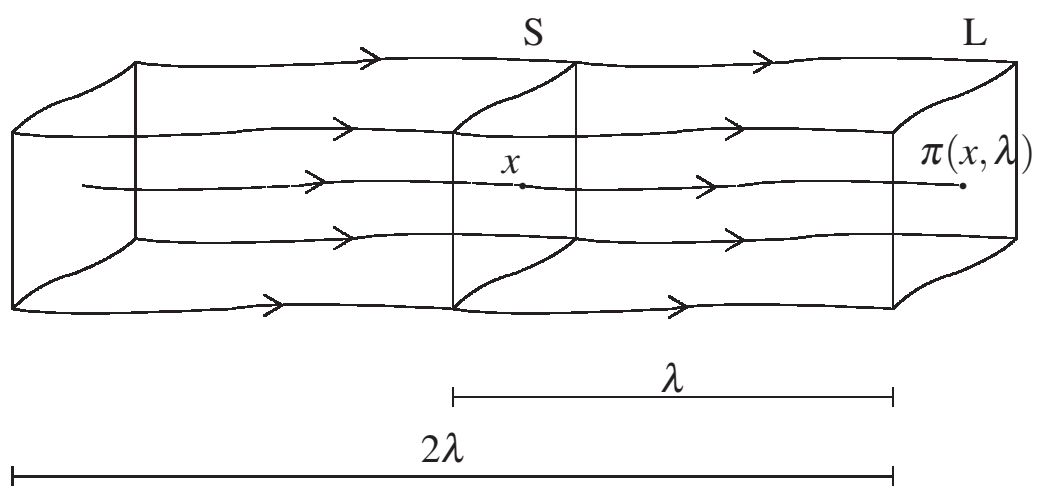

Figura 1.3: $\lambda$-tubo $F(L,[0,2 \lambda])$.

Lema 1.2.2 [16, Lema 1.9] Seja $(X, \pi)$ um sistema semidinâmico. Se $S$ é uma $\lambda$-seção através de $x, x \in X$, e $\mu \leq \lambda$, então $S$ também é uma $\mu$-seção através de $x$.

Na próxima definição, apresentamos as condições TC e STC para um tubo. 
Definição 1.2.3 Seja $(X, \pi)$ um sistema semidinâmico. Qualquer tubo $F(L,[0,2 \lambda])$ dado pela seção $S$ através de $x \in X$ tal que

$$
S \subset M \cap F(L,[0,2 \lambda])
$$

é chamado um TC-tubo através de $x$. Dizemos que um ponto $x \in M$ satisfaz a condição de tubo e escrevemos abreviadamente (TC), se existe um TC-tubo, $F(L,[0,2 \lambda])$, através de $x$.

Se $S=M \cap F(L,[0,2 \lambda])$, dizemos que $F(L,[0,2 \lambda])$ é um STC-tubo através de $x$. Dizemos que um ponto $x \in M$ satisfaz a condição forte de tubo e escrevemos abreviadamente (STC), se existe um STC-tubo, $F(L,[0,2 \lambda])$, através de $x$.

Exemplo 1.2.4 [17, Exemplo 2.5] Consideremos o sistema semidinâmico em $\mathbb{R}^{2}$ dado por $\pi((x, y), t)=$ $(x+t, y)$ e $M=\left\{(x, y) \in \mathbb{R}^{2}: x=0\right\} \cup\left\{(x, y) \in \mathbb{R}^{2}: x=y, x \geq 0\right\}$, veja a Figura 1.4. O ponto $(0,0)$ satisfaz a condição TC mas não satisfaz a condição STC.

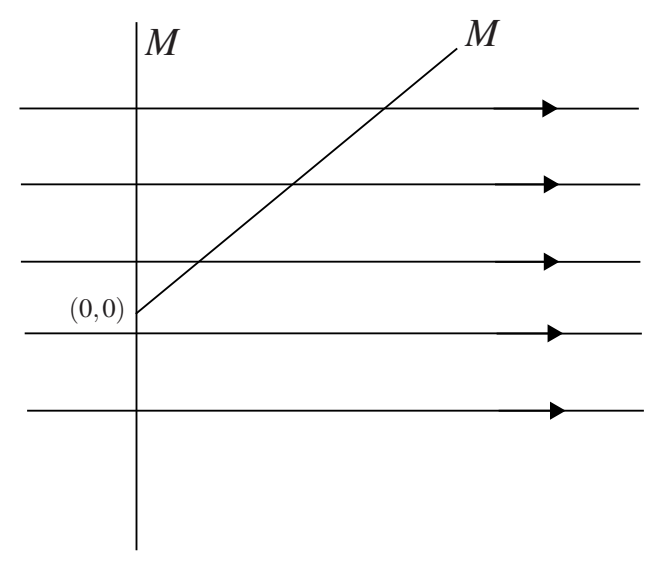

Figura 1.4: $(0,0)$ satisfaz a condição TC.

O próximo resultado é consequência do Lema 1.2.2 e das definições acima. O leitor pode encontrar a demonstração em [17].

Lema 1.2.5 [17, Lema 3.3] Seja $(X, \pi ; M, I)$ um sistema semidinâmico impulsivo. Suponhamos que exista um ponto $x \in X$ que satisfaça a condição $T C$ (STC) com uma $\lambda$-seção $S$. Então, para qualquer $\eta<\lambda$, o conjunto $S$ também será uma $\eta$-seção com um TC-tubo (STC-tubo).

Definição 1.2.6 Uma função $f: X \rightarrow \mathbb{R}$ é denominada semicontínua superiormente em um ponto $a \in X$ quando, para cada $\varepsilon>0$ dado, existe $\delta>0$ tal que se $x \in X$ e $d(x, a)<\delta$ então 
$f(x)<f(a)+\varepsilon$. Dizemos que $f: X \rightarrow \mathbb{R}$ é semicontínua superiormente quando ela o for em cada ponto de $X$. Analogamente, $f$ é chamada semicontínua inferiormente em um ponto $a \in X$ quando, para cada $\varepsilon>0$ dado, existe $\delta>0$ tal que se $x \in X$ e $d(x, a)<\delta$ então $f(x)>f(a)-\varepsilon$. Em termos de sequências, $f$ é uma função semicontínua superiormente em $a \in X$ se, para toda sequência $\left\{x_{n}\right\}_{n \geq 1} \subset X, \operatorname{com} x_{n} \stackrel{n \rightarrow+\infty}{\longrightarrow} a$, tivermos $\limsup f\left(x_{n}\right) \leq f(a)$. De mesma forma, $f$ é uma função semicontínua inferiormente em $a \in X$ se, para toda sequência $\left\{x_{n}\right\}_{n \geq 1} \subset X$, com $x_{n} \stackrel{n \rightarrow+\infty}{\longrightarrow} a$, tivermos $\liminf _{n \rightarrow+\infty} f\left(x_{n}\right) \geq f(a)$. Dizemos, então, que $f: X \rightarrow \mathbb{R}$ é contínua em um ponto $a \in X$ se, e somente se, ela for contínua superiormente e inferiormente no ponto $a$.

Exemplo 1.2.7 [17, Exemplo 2.6] Consideremos o sistema semidinâmico em $\mathbb{R}$ dado por $\pi(x, t)=$ $x+t$. Assim, consideremos o sistema impulsivo associado com $M=\mathbb{N}$ e $I(n)=n+\frac{1}{2}$ para cada $n \in \mathbb{N}$. Então

$$
\phi(x)= \begin{cases}-x & \text { se } \quad x<0 \\ 1+E(x)-x & \text { se } x \geq 0\end{cases}
$$

onde $E(x)$ denota a parte inteira de $x$. Notemos que a função $\phi$ não é semicontínua inferiormente nos pontos pertencentes ao conjunto $\mathbb{N}$. Com efeito, dado $x \in \mathbb{N}$, consideremos a sequência dada por $x_{n}=x-\frac{1}{n}$ para todo $n \in \mathbb{N}^{*}$. Então $x_{n} \stackrel{n \rightarrow+\infty}{\longrightarrow} x \mathrm{e}$

$$
\liminf _{n \rightarrow+\infty} \phi\left(x_{n}\right)=\liminf _{n \rightarrow+\infty}[1+(x-1)-(x-1 / n)]=0<\phi(x)=1 .
$$

Notemos que todo elemento $x \in \mathbb{N}$ satisfaz a condição STC.

O teorema seguinte diz que, se $x \notin M$, então $\phi$ é semicontínua inferiormente em $x$.

Teorema 1.2.8 [17, Teorema 3.5] Seja $(X, \pi ; M, I)$ um sistema semidinâmico impulsivo. Então, a função $\phi$ é semicontínua inferiormente em qualquer $x \in X \backslash M$.

Demonstração: Sejam $x \in X \backslash M$ e $\left\{x_{n}\right\}_{n \geq 1} \subset X$ uma sequência qualquer tal que $x_{n} \stackrel{n \rightarrow+\infty}{\longrightarrow} x$. Suponhamos que $\phi(x)=+\infty$. Se existir uma subsequência $\left\{x_{n_{k}}\right\}_{k \geq 1}$ tal que $\left\{\phi\left(x_{n_{k}}\right)\right\}_{k \geq 1}$ converge para algum número real $\lambda \geq 0$, então

$$
\pi\left(x_{n_{k}}, \phi\left(x_{n_{k}}\right)\right) \stackrel{k \rightarrow+\infty}{\longrightarrow} \pi(x, \lambda) \in M
$$


pois $\pi\left(x_{n_{k}}, \phi\left(x_{n_{k}}\right)\right) \in M$ para todo $k=1,2, \ldots$ e $M$ é fechado. Logo $\phi(x) \leq \lambda$ o que é uma contradição. Portanto $\liminf _{n \rightarrow+\infty} \phi\left(x_{n}\right)=+\infty=\phi(x)$.

Agora, vamos assumir que $\phi(x)=c \in(0,+\infty)$. Suponhamos que $\phi\left(x_{n}\right) \stackrel{n \rightarrow+\infty}{\longrightarrow} t$. Como $x \notin M$ então, para $n \in \mathbb{N}$ suficientemente grande, temos $x_{n} \notin M$. Entretanto $\pi\left(x_{n}, \phi\left(x_{n}\right)\right) \in M$ e, como $M$ é fechado e $\pi$ é contínua, segue que

$$
\pi\left(x_{n}, \phi\left(x_{n}\right)\right) \stackrel{n \rightarrow+\infty}{\longrightarrow} \pi(x, t) \in M
$$

Logo $c=\phi(x) \leq t$. Portanto $\phi$ é semicontínua inferiormente em $x$.

Se $x \in M$ e $x$ não é um ponto inicial, então $\phi$ não é semicontínua inferiormente em $x$. O próximo resultado mostra este fato.

Teorema 1.2.9 [17, Proposição 3.6] Seja $(X, \pi ; M, I)$ um sistema semidinâmico impulsivo e suponhamos que $x \in M$ não seja um ponto inicial. Então a função $\phi$ não é semicontínua inferiormente em $x$.

Demonstração: Seja $x \in M$ e suponhamos que $x$ não seja um ponto inicial. Então existem $\varepsilon>0 \mathrm{e}$ $y \in X$ tais que $\pi(y, \varepsilon)=x$. Podemos supor $\pi(y,[0, \varepsilon)) \cap M=\emptyset$. Agora, consideremos uma sequência crescente de números reais $\left\{\varepsilon_{n}\right\}_{n \geq 1}$ tal que $\varepsilon_{n}>0$ para todo $n \in \mathbb{N}^{*}$ e $\varepsilon_{n} \stackrel{n \rightarrow+\infty}{\longrightarrow} \varepsilon$. Definindo $y_{n}=$ $\pi\left(y, \varepsilon_{n}\right)$ para cada $n \in \mathbb{N}^{*}$, temos que $y_{n} \stackrel{n \rightarrow+\infty}{\longrightarrow} x \mathrm{e}$

$$
\phi\left(y_{n}\right)=\phi\left(\pi\left(y, \varepsilon_{n}\right)\right)=\varepsilon-\varepsilon_{n} \stackrel{n \rightarrow+\infty}{\longrightarrow} 0<\phi(x) .
$$

Portanto $\phi$ não é semicontínua inferiormente em $x$.

No Teorema 1.2.10 a seguir, estabelecemos condições suficientes para que $\phi$ seja semicontínua superiormente em pontos de $X$.

Teorema 1.2.10 [17, Teorema 3.4] Se, em um sistema semidinâmico impulsivo $(X, \pi ; M, I)$, cada ponto pertencente ao conjunto impulsivo $M$ satisfaz a condição (TC) então $\phi$ é semicontínua superiormente em $X$.

Demonstração: Seja $x \in X$. Podemos assumir que $\phi(x)=u \in(0,+\infty)$. Neste caso, $\pi(x, u)=y \in M$ e $\pi(x,(0, u)) \cap M=\emptyset$. Pelo Lema 1.2.5, podemos tomar $\varepsilon<u$ tal que $U=F(L,[0,2 \varepsilon])$ seja um 
TC-tubo com uma $\varepsilon$-seção através de $y$ igual a $F(L, \varepsilon)$. Então existe uma vizinhança $V$ de $x$ tal que $\pi(V, u) \subset U$. Assim, $\pi(z, u) \in F(L,[0,2 \varepsilon])$, para qualquer $z \in V$. Além disso, dado qualquer $z \in V$, existe um $\eta_{z} \in[0,2 \varepsilon]$ tal que $\pi\left(z, u+\eta_{z}\right) \in L$. Como $\varepsilon<u$, segue que $u+\eta_{z}-\varepsilon>0$ e

$$
\pi\left(z, u+\eta_{z}-\varepsilon\right) \in F(L, \varepsilon)=S \subset M
$$

Daí, $\phi(z) \leq u+\eta_{z}-\varepsilon<u+\varepsilon=\phi(x)+\varepsilon$. Portanto $\phi$ é semicontínua superiormente em $x$.

O próximo resultado diz respeito à continuidade da função $\phi$.

Teorema 1.2.11 [17, Teorema 3.8] Consideremos um sistema semidinâmico impulsivo $(X, \pi ; M, I)$. Suponhamos que nenhum ponto inicial pertença ao conjunto impulsivo $M$ e que cada elemento de M satisfaça a condição (TC). Então $\phi$ é contínua em $x$ se, e somente se, $x \in X \backslash M$.

Demonstração: $(\Rightarrow)$ Suponhamos que $x \in M$. Como $x$ não é ponto inicial, segue, pelo Teorema 1.2.9, que $\phi$ não é semicontínua inferiormente. Mas isto é um absurdo. Portanto $x \in X \backslash M$.

$(\Leftarrow)$ Seja $x \in X \backslash M$. Pelo Teorema 1.2.8, $\phi$ é semicontínua inferiormente em $x$. E como cada ponto de $M$ satisfaz a condição (TC), pelo Teorema 1.2.10, $\phi$ é semicontínua superiormente em $x$. Portanto $\phi$ é contínua em $x$.

Observação 1.2.12 Vamos assumir em todo este trabalho que o conjunto impulsivo $M$ satisfaz a condição STC e que não existe ponto inicial em $M$. Assim, $\phi$ é semicontínua superiormente em $X$ e contínua em $X \backslash M$.

\subsection{Invariância}

O conceito de invariância positiva para sistemas semidinâmicos com impulsos é definido semelhante ao caso contínuo, veja a próxima definição.

Definição 1.3.1 Sejam $(X, \pi ; M, I)$ um sistema semidinâmico impulsivo e $A$ um subconjunto de $X$.

Dizemos que $A$ é positivamente $\tilde{\pi}$-invariante se $\widetilde{\pi}^{+}(A)=\cup\left\{\widetilde{\pi}^{+}(x): x \in A\right\} \subset A$. Ainda, dizemos que $A$ é $I$-invariante se $I(x) \in A$ para todo $x \in M \cap A$. 
Notemos que a órbita positiva impulsiva $\widetilde{\pi}^{+}(x)$ é positivamente $\tilde{\pi}$-invariante para todo $x \in X$.

O seguinte exemplo, descrito em [18], mostra que em geral, não existe relação entre $\pi$-invariância, $\tilde{\pi}$-invariância e $I$-invariância.

Exemplo 1.3.2 Consideremos o sistema semidinâmico impulsivo em $\mathbb{R}$ dado pelo sistema semidinâmico $\pi(x, t)=t+x, M=\{1\}$ e $I(1)=-1$. Então o conjunto $A=[0,+\infty)$ é positivamente $\pi$-invariante mas não é positivamente $\tilde{\pi}$-invariante e nem $I$-invariante. Entretanto, o conjunto $B=[-1,1)$ é positivamente $\widetilde{\pi}$-invariante mas não é positivamente $\pi$-invariante. $\mathrm{O}$ conjunto $C=[-2,2]$ é $I$-invariante mas não é positivamente $\widetilde{\pi}$-invariante. Já o conjunto $D=[1,+\infty)$ é positivamente $\widetilde{\pi}$-invariante mas não é $I$-invariante.

A seguir, apresentamos três resultados sobre invariância.

Proposição 1.3.3 [18, Proposição 2.1] Sejam $(X, \pi ; M, I)$ um sistema semidinâmico impulsivo e A um subconjunto de X positivamente $\pi$-invariante e I-invariante. Então A é positivamente $\tilde{\pi}$-invariante.

Demonstração: Seja $x \in A$. Então $\widetilde{\pi}(x,[0, \phi(x)))=\pi(x,[0, \phi(x))) \subset A$ e $x_{1}=\pi(x, \phi(x)) \in A \cap$ M. Como $A$ é $I$-invariante, segue que $x_{1}^{+}=I\left(x_{1}\right) \in A$. Logo, $\tilde{\pi}(x,[0, \phi(x)]) \subset A$. Do mesmo modo, $\tilde{\pi}\left(x,\left[\phi(x), \phi(x)+\phi\left(x_{1}^{+}\right)\right)\right)=\pi\left(x_{1}^{+},\left[0, \phi\left(x_{1}^{+}\right)\right)\right) \subset A$ e $x_{2}=\pi\left(x_{1}^{+}, \phi\left(x_{1}^{+}\right)\right) \in A \cap M . \quad$ Daí $x_{2}^{+}=I\left(x_{2}\right) \in A$ e $\tilde{\pi}\left(x,\left[\phi(x), \phi(x)+\phi\left(x_{1}^{+}\right)\right]\right) \subset A$. Continuando com este processo segue que $\tilde{\pi}^{+}(x) \subset A$.

Proposição 1.3.4 [18, Proposição 2.2] $\operatorname{Sejam~}(X, \pi ; M, I)$ um sistema semidinâmico impulsivo e A um subconjunto fechado de X positivamente $\tilde{\pi}$-invariante. Então A é positivamente $\pi$-invariante.

Demonstração: Suponhamos que $A$ não seja positivamente $\pi$-invariante, isto é, existem $x \in A$ e $\mu \in \mathbb{R}_{+}$tais que $\pi(x, \mu) \notin A$. Definamos $t=\inf \{s>0: \pi(x, s) \notin A\}$. Observemos que $t>0$, pois $\tilde{\pi}(x,[0, \phi(x)))=\pi(x,[0, \phi(x))) \subset A$. Como $\pi(x,[0, t)) \subset A$, segue que $\pi(x, t) \in \bar{A}=A$. Assim

$$
\pi(\pi(x, t),[0, \phi(\pi(x, t))))=\tilde{\pi}(\pi(x, t),[0, \phi(\pi(x, t)))) \subset A,
$$

isto é,

$$
\pi(x,[t, t+\phi(\pi(x, t)))) \subset A
$$


e isto contradiz a definição de $t$. Portanto $A$ é positivamente $\pi$-invariante.

Proposição 1.3.5 [18, Teorema 2.5] Sejam $(X, \pi ; M, I)$ um sistema semidinâmico impulsivo e A um subconjunto compacto e positivamente $\tilde{\pi}$-invariante de X. Se E é uma componente conexa de A I-invariante, então E é positivamente $\tilde{\pi}$-invariante.

Demonstração: Seja $x \in E$, então $\tilde{\pi}(x,[0, \phi(x)))=\pi(x,[0, \phi(x))) \subset A$. Pela continuidade de $\pi$ e conexidade de $E$ temos $\pi(x,[0, \phi(x))) \subset E$. Se $\phi(x)=+\infty$ concluímos a prova. Do contrário se $\phi(x)<+\infty$, então $x_{1}=\pi(x, \phi(x)) \in \bar{E}=E$ e $x_{1}^{+}=I\left(x_{1}\right) \in E$, pois $E$ é $I$-invariante. Assim $\tilde{\pi}\left(x,\left[\phi(x), \phi(x)+\phi\left(x_{1}^{+}\right)\right)\right)=\pi\left(x_{1}^{+},\left[0, \phi\left(x_{1}^{+}\right)\right)\right) \subset E$. Se $\phi\left(x_{1}^{+}\right)=+\infty$ concluímos a prova. Do contrário se $\phi\left(x_{1}^{+}\right)<+\infty$, então $x_{2}=\pi\left(x_{1}^{+}, \phi\left(x_{1}^{+}\right)\right) \in \bar{E}=E$ e $x_{2}^{+}=I\left(x_{2}\right) \in E$. Continuando com este processo concluímos que $\tilde{\pi}^{+}(x) \subset E$.

\subsection{Conjuntos limite}

Definição 1.4.1 Seja $(X, \pi ; M, I)$ um sistema semidinâmico impulsivo. Para cada $x \in X$ definimos o conjunto limite positivo de $x$ com respeito a $\tilde{\pi}$ pelo conjunto

$$
\begin{gathered}
\widetilde{L}^{+}(x)=\left\{y \in X \text { : existe uma sequência }\left\{t_{n}\right\}_{n \geq 1} \subset \mathbb{R}_{+}\right. \text {tal que } \\
\left.t_{n} \stackrel{n \rightarrow+\infty}{\longrightarrow}+\infty \text { e } \tilde{\pi}\left(x, t_{n}\right) \stackrel{n \rightarrow+\infty}{\longrightarrow} y\right\} .
\end{gathered}
$$

e o prolongamento do conjunto limite positivo de $x$ com respeito a $\tilde{\pi}$ é dado por

$$
\begin{gathered}
\widetilde{J}^{+}(x)=\left\{y \in X: \text { existem sequências }\left\{x_{n}\right\}_{n \geq 1} \subset X \text { e }\left\{t_{n}\right\}_{n \geq 1} \subset \mathbb{R}_{+}\right. \\
\text {tais que } \left.x_{n} \stackrel{n \rightarrow+\infty}{\longrightarrow} x, \quad t_{n} \stackrel{n \rightarrow+\infty}{\longrightarrow}+\infty \text { e } \tilde{\pi}\left(x_{n}, t_{n}\right) \stackrel{n \rightarrow+\infty}{\longrightarrow} y\right\} .
\end{gathered}
$$

Podemos caracterizar os conjuntos $\widetilde{L}^{+}(x)$ e $\widetilde{J}^{+}(x)$ da seguinte maneira.

Lema 1.4.2 [11, Lema 3.2 e Lema 3.27] Sejam $(X, \pi ; M, I)$ um sistema semidinâmico impulsivo e 
$x \in X$. Então

$$
\widetilde{L}^{+}(x)=\bigcap_{t>0} \overline{\tilde{\pi}(x,[t,+\infty))} \quad e \quad \widetilde{J}^{+}(x)=\bigcap_{\varepsilon>0} \bigcap_{\bigcup\{\tilde{\pi}(B(x, \varepsilon), \tau): \tau \geq t\}}
$$

Decorre do Lema 1.4.2 o seguinte resultado.

Proposição 1.4.3 Os conjuntos $\widetilde{L}^{+}(x)$ e $\widetilde{J}^{+}(x), x \in X$, são fechados em $X$.

Ao contrário do caso contínuo, o conjunto limite positivo de um ponto $x \in X$ pode não ser positivamente $\tilde{\pi}$-invariante. Mostremos este fato no exemplo seguinte.

Exemplo 1.4.4 Consideremos o sistema semidinâmico impulsivo em $\mathbb{R}$ dado pelo sistema semidinâmico $\pi(x, t)=t+x, M=\{1\}$ e $I(1)=-1$. Note que $\widetilde{L}^{+}(0)=[-1,1]$. Entretanto, $\tilde{\pi}(1, t)=$ $\pi(1, t) \in[1,+\infty)$ para cada $t \geq 0$. Assim $\widetilde{L}^{+}(0)$ não é positivamente $\tilde{\pi}$-invariante.

Na sequência, apresentamos um resultado sobre convergência em sistemas impulsivos. Após esse resultado, estabelecemos condições para obtermos invariância dos conjuntos limite.

Lema 1.4.5 [23, Lema 2.3][6, Lema 3.2] Sejam $(X, \pi ; M, I)$ um sistema semidinâmico impulsivo e $x \in X \backslash M$. Suponhamos que $I(M) \cap M=\emptyset$. Seja $\left\{z_{n}\right\}_{n \geq 1}$ uma sequência em $X$ tal que $z_{n} \stackrel{n \rightarrow+\infty}{\longrightarrow} x$. Dado $t \geq 0$, existe uma sequência $\left\{\varepsilon_{n}\right\}_{n \geq 1} \subset \mathbb{R}$ tal que $\varepsilon_{n} \stackrel{n \rightarrow+\infty}{\longrightarrow} 0$ e $\tilde{\pi}\left(z_{n}, t+\varepsilon_{n}\right) \stackrel{n \rightarrow+\infty}{\longrightarrow} \tilde{\pi}(x, t)$.

Demonstração: Se $\phi(x)=+\infty$, segue pela continuidade da $\phi$ sobre $X \backslash M$, que dado $t \in[0,+\infty)$ existe um natural $n_{0}$ tal que, para $n \geq n_{0}$, temos $\phi\left(z_{n}\right)>t$. Consequentemente, para $n \geq n_{0}$, $\tilde{\pi}\left(z_{n}, t\right)=\pi\left(z_{n}, t\right)$ e o resultado segue da continuidade de $\pi$.

Agora, suponhamos que $\phi(x)<+\infty$. Então, já que $\phi$ é contínua em $X \backslash M$, podemos assumir que $\phi\left(z_{n}\right)<+\infty$ para todo natural $n=1,2,3, \ldots$

Temos três casos a considerar.

Caso 1: $0 \leq t<\phi(x)$.

Neste caso, seja $0<\varepsilon<\phi(x)-t$. Segue da continuidade de $\phi$ em $X \backslash M$, que existe $n_{0} \in \mathbb{N}$ tal que $\phi(x)-\varepsilon<\phi\left(z_{n}\right)$ para todo $n \geq n_{0}$. Logo $t<\phi\left(z_{n}\right)$ e $\tilde{\pi}\left(z_{n}, t\right)=\pi\left(z_{n}, t\right)$ para $n \geq n_{0}$. Tomando 
$\varepsilon_{n}=0, n=1,2, \ldots$, segue que

$$
\tilde{\pi}\left(z_{n}, t+\varepsilon_{n}\right)=\pi\left(z_{n}, t\right) \stackrel{n \rightarrow+\infty}{\longrightarrow} \pi(x, t)=\tilde{\pi}(x, t) .
$$

Caso 2: $t=\phi(x)$.

$\operatorname{Temos} \tilde{\pi}(x, t)=\tilde{\pi}(x, \phi(x))=x_{1}^{+}$. Note que

$$
\left(z_{n}\right)_{1}=\pi\left(z_{n}, \phi\left(z_{n}\right)\right) \stackrel{n \rightarrow+\infty}{\longrightarrow} \pi(x, \phi(x))=x_{1} .
$$

Mas como I é contínua, então

$$
I\left(\left(z_{n}\right)_{1}\right) \stackrel{n \rightarrow+\infty}{\longrightarrow} I\left(x_{1}\right)
$$

isto é, $\left(z_{n}\right)_{1}^{+} \stackrel{n \rightarrow+\infty}{\longrightarrow} x_{1}^{+}$. Pela continuidade de $\phi$ em $X \backslash M$, segue que $\left|\phi\left(z_{n}\right)-\phi(x)\right| \stackrel{n \rightarrow+\infty}{\longrightarrow} 0$. Logo $\phi\left(z_{n}\right)=t+\varepsilon_{n}$, onde $\left\{\varepsilon_{n}\right\}_{n \geq 1}$ é uma sequência de números reais com $\varepsilon_{n} \stackrel{n \rightarrow+\infty}{\longrightarrow} 0$, daí

$$
\tilde{\pi}\left(z_{n}, t+\varepsilon_{n}\right)=\tilde{\pi}\left(z_{n}, \phi\left(z_{n}\right)\right)=\left(z_{n}\right)_{1}^{+} \stackrel{n \rightarrow+\infty}{\longrightarrow} x_{1}^{+}=\tilde{\pi}(x, t) .
$$

Caso 3: $t>\phi(x)$.

Neste caso, existe $m \in\{1,2,3,4, \ldots\}$ tal que $t=\sum_{i=0}^{m-1} \phi\left(x_{i}^{+}\right)+t^{\prime} \operatorname{com} 0 \leq t^{\prime}<\phi\left(x_{m}^{+}\right)$. Definamos $\left\{\left(z_{n}\right)_{i}\right\}_{i \geq 1}$ indutivamente por

$$
\left(z_{n}\right)_{1}=\pi\left(z_{n}, \phi\left(z_{n}\right)\right) \text { e }\left(z_{n}\right)_{i+1}=\pi\left(\left(z_{n}\right)_{i}^{+}, \phi\left(\left(z_{n}\right)_{i}^{+}\right)\right), \quad i \in\{1,2,3,4, \ldots\} .
$$

Seja $t_{n}=\sum_{i=0}^{m-1} \phi\left(\left(z_{n}\right)_{i}^{+}\right)$. Então, como $\phi\left(z_{n}\right) \stackrel{n \rightarrow+\infty}{\longrightarrow} \phi(x)$, temos

$$
\left(z_{n}\right)_{1}=\pi\left(z_{n}, \phi\left(z_{n}\right)\right) \stackrel{n \rightarrow+\infty}{\longrightarrow} \pi(x, \phi(x))=x_{1} .
$$

Segue da continuidade de $I$ que $I\left(\left(z_{n}\right)_{1}\right) \stackrel{n \rightarrow+\infty}{\longrightarrow} I\left(x_{1}\right)$, isto é, $\left(z_{n}\right)_{1}^{+} \stackrel{n \rightarrow+\infty}{\longrightarrow} x_{1}^{+}$. Como 
$\phi\left(\left(z_{n}\right)_{1}^{+}\right) \stackrel{n \rightarrow+\infty}{\longrightarrow} \phi\left(x_{1}^{+}\right)$, pois $x_{1}^{+} \notin M$, obtemos

$$
\left(z_{n}\right)_{2}=\pi\left(\left(z_{n}\right)_{1}^{+}, \phi\left(\left(z_{n}\right)_{1}^{+}\right)\right) \stackrel{n \rightarrow+\infty}{\longrightarrow} \pi\left(x_{1}^{+}, \phi\left(x_{1}^{+}\right)\right)=x_{2} .
$$

Prosseguindo desta maneira, obtemos $\left(z_{n}\right)_{i} \stackrel{n \rightarrow+\infty}{\longrightarrow} x_{i}$, para todo $i=1,2,3 \ldots$. Pela continuidade de $I$, segue que

$$
\left(z_{n}\right)_{i}^{+} \stackrel{n \rightarrow+\infty}{\longrightarrow} x_{i}^{+}
$$

para todo $i=1,2,3, \ldots$ Assim $\sum_{i=0}^{m-1} \phi\left(\left(z_{n}\right)_{i}^{+}\right) \stackrel{n \rightarrow+\infty}{\longrightarrow} \sum_{i=0}^{m-1} \phi\left(x_{i}^{+}\right)$. Defina a sequência $\left\{\varepsilon_{n}\right\}_{n \geq 1}$ por $\varepsilon_{n}=t_{n}+t^{\prime}-t$. Notemos que $\varepsilon_{n} \stackrel{n \rightarrow+\infty}{\longrightarrow} 0$. Então

$$
\tilde{\pi}\left(z_{n}, t+\varepsilon_{n}\right)=\pi\left(\left(z_{n}\right)_{m}^{+}, t^{\prime}\right) \stackrel{n \rightarrow+\infty}{\longrightarrow} \pi\left(x_{m}^{+}, t^{\prime}\right)=\tilde{\pi}(x, t)
$$

O lema está provado.

Se $t \geq 0$ for tomado de tal forma que $\tilde{\pi}(x, t) \neq x_{j}^{+}=I\left(x_{j}\right)$ para todo $j=1,2,3, \ldots$, então a convergência no Lema 1.4.5 não dependerá da sequência $\left\{\varepsilon_{n}\right\}_{n \geq 1}$, ou seja, $\tilde{\pi}\left(z_{n}, t\right) \stackrel{n \rightarrow+\infty}{\longrightarrow} \tilde{\pi}(x, t)$ sempre que $t \neq \sum_{j=0}^{k} \phi\left(x_{j}^{+}\right), k=0,1,2, \ldots$. Veja o próximo resultado.

Lema 1.4.6 [9, Lema 3.3] Sejam $(X, \pi ; M, I)$ um sistema semidinâmico impulsivo e $x \in X \backslash M$. Suponha que $I(M) \cap M=\emptyset$. Se $\left\{z_{n}\right\}_{n \geq 1}$ é uma sequência em $X$ que converge para $x$, então dado $t \geq 0$ tal que $t \neq \sum_{j=0}^{k} \phi\left(x_{j}^{+}\right)$para todo $k=0,1,2, \ldots$, temos $\tilde{\pi}\left(z_{n}, t\right) \stackrel{n \rightarrow+\infty}{\longrightarrow} \tilde{\pi}(x, t)$.

Teorema 1.4.7 [13, Proposição 4.1] Seja $(X, \pi ; M, I)$ um sistema semidinâmico impulsivo. Suponhamos que $I(M) \cap M=\emptyset$. Então $\widetilde{L}^{+}(x) \backslash M$ é positivamente $\widetilde{\pi}$-invariante para todo $x \in X$.

Demonstração: Sejam $y \in \widetilde{L}^{+}(x) \backslash M$ e $t \geq 0$ arbitrário. Então existe uma sequência $\left\{t_{n}\right\}_{n \geq 1} \subset \mathbb{R}_{+} \operatorname{com} t_{n} \stackrel{n \rightarrow+\infty}{\longrightarrow}+\infty$ tal que $\tilde{\pi}\left(x, t_{n}\right) \stackrel{n \rightarrow+\infty}{\longrightarrow} y$. Como $y \notin M$, podemos supor que $\left\{\tilde{\pi}\left(x, t_{n}\right)\right\}_{n \geq 1} \subset X \backslash M$. Então pelo Lema 1.4.5, existe uma sequência $\left\{\varepsilon_{n}\right\}_{n \geq 1} \subset \mathbb{R}$ com $\varepsilon_{n} \stackrel{n \rightarrow+\infty}{\longrightarrow} 0$ tal que $\tilde{\pi}\left(x, t_{n}+t+\varepsilon_{n}\right)=\tilde{\pi}\left(\tilde{\pi}\left(x, t_{n}\right), t+\varepsilon_{n}\right) \stackrel{n \rightarrow+\infty}{\longrightarrow} \tilde{\pi}(y, t)$. Notemos que $\left\{t_{n}+t+\varepsilon_{n}\right\}_{n \geq 1} \subset \mathbb{R}_{+}$com $t_{n}+t+\varepsilon_{n} \stackrel{n \rightarrow+\infty}{\longrightarrow}+\infty$. Logo $\tilde{\pi}(y, t) \in \widetilde{L}^{+}(x) \backslash M$ já que $I(M) \cap M=\emptyset$. Como $t \geq 0$ foi tomado de maneira arbitrária, o teorema está provado. 
Corolário 1.4.8 [11, Lema 3.5] Sejam $(X, \pi ; M, I)$ um sistema semidinâmico impulsivo e $x \in X$. Suponhamos que $I(M) \cap M=\emptyset$. Se $\widetilde{L}^{+}(x) \cap M=\emptyset$, então $\widetilde{L}^{+}(x)$ é positivamente $\widetilde{\pi}$-invariante.

Para os conjuntos $\widetilde{J}^{+}(x)$ e $\widetilde{D}^{+}(x), x \in X$, temos o seguinte resultado sobre invariância.

Teorema 1.4.9 [12, Teorema 2.4.8] Seja $(X, \pi ; M, I)$ um sistema semidinâmico impulsivo. Se $I(M) \cap M=\emptyset$, então os conjuntos $\widetilde{D}^{+}(x) \backslash M$ e $\widetilde{J}^{+}(x) \backslash M$ são positivamente $\tilde{\pi}$-invariantes para todo $x \in X$.

Demonstração: A prova é análoga a demonstração do Teorema 1.4.7.

Corolário 1.4.10 [11, Lema 3.27] Sejam $(X, \pi ; M, I)$ um sistema semidinâmico impulsivo e $x \in X$. Suponhamos que $I(M) \cap M=\emptyset$.

a) Se $\widetilde{J}^{+}(x) \cap M=\emptyset$, então $\widetilde{J}^{+}(x)$ é positivamente $\widetilde{\pi}$-invariante;

b) Se $\widetilde{D}^{+}(x) \cap M=\emptyset$, então $\widetilde{D}^{+}(x)$ é positivamente $\tilde{\pi}$-invariante.

No que segue, apresentamos uma caracterização para o fecho de uma órbita positiva em um sistema impulsivo $(X, \pi ; M, I)$.

Lema 1.4.11 [9, Lema 3.1] Sejam $(X, \pi ; M, I)$ um sistema semidinâmico impulsivo e $x \in X$. Suponha que $\phi\left(x_{j}^{+}\right)<+\infty$ para todo $j=0,1,2, \ldots$ então

$$
\overline{\widetilde{\pi}^{+}(x)}=\tilde{\pi}^{+}(x) \cup \widetilde{L}^{+}(x) \cup\left\{x_{j}: j=1,2, \ldots\right\},
$$

onde $x_{j}=\pi\left(x_{j-1}^{+}, \phi\left(x_{j-1}^{+}\right)\right), j=1,2, \ldots$. Note que se $\phi\left(x_{j}^{+}\right)<+\infty$ para cada $j=0,1, \ldots, k$ e $\phi\left(x_{k+1}^{+}\right)=+\infty$, então

$$
\overline{\tilde{\pi}^{+}(x)}=\widetilde{\pi}^{+}(x) \cup \widetilde{L}^{+}(x) \cup\left\{x_{j}: j=1,2, \ldots, k+1\right\} .
$$




\subsection{Hipóteses gerais}

Em todo este trabalho vamos considerar as seguintes hipóteses:

(H1) O conjunto $M$ satisfaz a condição STC e não existem pontos iniciais em $M$. Assim $\phi$ é contínua em $X \backslash M$, veja Teorema 1.2.11.

(H2) $M \cap I(M)=\emptyset$.

(H3) $\tilde{\pi}(x, t)$ está definida para todo $t \geq 0$, qualquer que seja $x \in X$. 


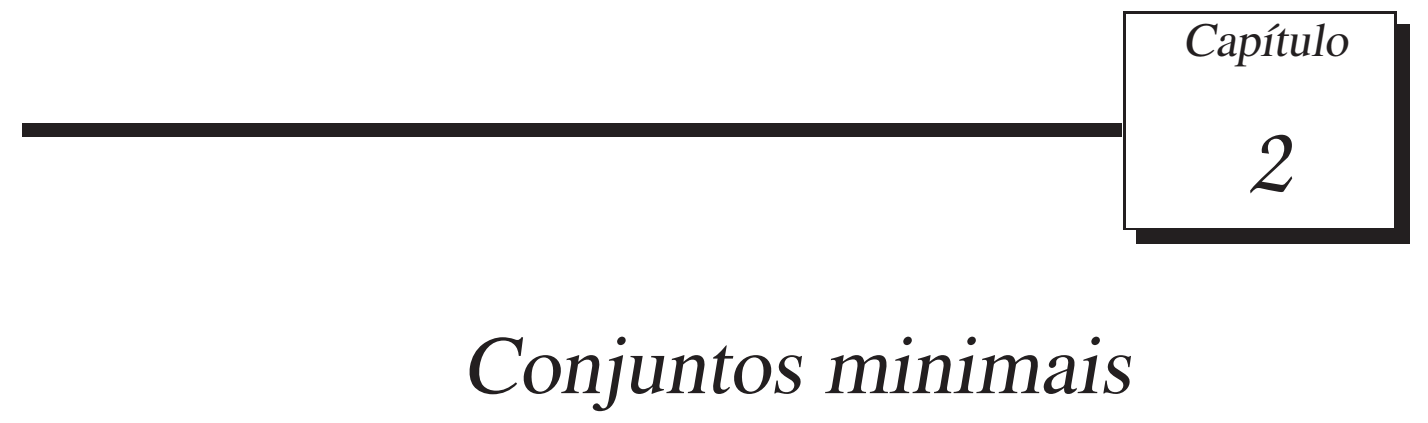

Neste capítulo, apresentamos a teoria de conjuntos minimais para sistemas semidinâmicos impulsivos. Os resultados desse capítulo estão apresentados no artigo [13].

\subsection{Conjuntos minimais}

Em [21], Kaul define o conceito de minimalidade para um conjunto $A$ em um sistema semidinâmico impulsivo $(X, \pi ; M, I)$ da seguinte forma:

$A$ é minimal em $(X, \pi ; M, I)$ se $A=\overline{\widetilde{\pi}^{+}(x)}$ para todo $x \in A \backslash M$.

Entretanto, na teoria clássica de sistemas dinâmicos contínuos, um conjunto $A$ em um sistema dinâmico $(X, \pi)$ é chamado minimal se $A$ é não vazio, fechado, invariante e ele não admite subconjunto próprio satisfazendo essas propriedades, veja [3, Definição 3.1(Capítulo III)]. Como consequência, segue que um conjunto não vazio $A \subset X$ é minimal se e somente se $\overline{\pi^{+}(x)}=A$ para todo $x \in A$, veja por exemplo, [3, Teorema 3.2 (Capítulo III)].

Desta forma, no que segue, definimos o conceito de minimalidade para sistemas semidinâmicos impulsivos de forma similar à definição clássica e mostramos que a definição dada por Kaul é equivalente à nossa definição.

Definição 2.1.1 Dizemos que um conjunto $A \subset X$ é minimal em $(X, \pi ; M, I)$ se as seguintes condições 
forem satisfeitas:

i) $A \backslash M \neq \emptyset$;

ii) A é fechado;

iii) $A \backslash M$ é positivamente $\widetilde{\pi}$ - invariante;

iv) A não admite subconjunto próprio satisfazendo as propriedades $i$ ), ii) e iii).

Exemplo 2.1.2 [13, Observação 4.1] Dado um sistema semidinâmico impulsivo $(X, \pi ; M, I)$, suponhamos que exista um ponto $x \in X \backslash M$ tal que $I(\pi(x, \phi(x)))=x$. Então $A=\pi(x,[0, \phi(x)])$ é um conjunto minimal em $(X, \pi ; M, I)$.

O próximo resultado mostra que a definição dada por Kaul é equivalente à Definição 5.4.1.

Teorema 2.1.3 [13, Teorema 4.1] $O$ conjunto $A \subset X$ é minimal em $(X, \pi ; M, I)$ se, e somente se, $A=\overline{\widetilde{\pi}^{+}(x)}$ para todo $x \in A \backslash M$.

Demonstração: Inicialmente, suponhamos que $A$ seja minimal. Seja $x \in A \backslash M$ arbitrário. Como $A \backslash M$ é positivamente $\widetilde{\pi}$ - invariante e $A$ é fechado, segue que

$$
\overline{\widetilde{\pi}^{+}(x)} \subset \bar{A}=A \text {. }
$$

Notemos que $\overline{\tilde{\pi}^{+}(x)} \backslash M \neq \emptyset, \overline{\tilde{\pi}^{+}(x)}$ é fechado e $\overline{\widetilde{\pi}^{+}(x)} \backslash M$ é positivamente $\tilde{\pi}$-invariante pelo Teorema 1.4.7 e pelo Lema 1.4.11. Como $A$ é minimal devemos ter $\overline{\widetilde{\pi}^{+}(x)}=A$.

Agora, vamos mostrar a condição suficiente. Seja $B \subset A$ tal que $B \backslash M \neq \emptyset, B$ é fechado e $B \backslash M$ é positivamente $\tilde{\pi}$-invariante. Dado $b \in B \backslash M$, segue que $b \in A \backslash M$. Pela hipótese temos que $A=\overline{\tilde{\pi}^{+}(b)}$. Como $B \backslash M$ é positivamente $\tilde{\pi}$-invariante, temos

$$
B \subset A=\overline{\widetilde{\pi}^{+}(b)} \subset B
$$

Então $A=B$. Assim $A$ não admite subconjunto próprio e $A$ é minimal. Isto mostra o teorema. 
O teorema a seguir mostra uma outra forma de caracterizar um conjunto minimal sob uma hipótese adicional.

Teorema 2.1.4 [13, Teorema 4.2] Seja $A \subset X$ e suponha que $\widetilde{L}^{+}(x) \backslash M \neq \emptyset$ para todo $x \in A$. Então A é minimal em $(X, \pi ; M, I)$ se, e somente se, $A=\widetilde{L}^{+}(x)$ para todo $x \in A \backslash M$.

Demonstração: Vamos mostrar a condição necessária. Seja $x \in A \backslash M$. Pelo Teorema 2.1.3 podemos escrever $\overline{\widetilde{\pi}^{+}(x)}=A$. Consequentemente

$$
\widetilde{L}^{+}(x) \subset A \text {. }
$$

Por outro lado, notemos que $\widetilde{L}^{+}(x) \backslash M \neq \emptyset, \widetilde{L}^{+}(x)$ é fechado e pelo Teorema 1.4.7 o conjunto $\widetilde{L}^{+}(x) \backslash M$ é positivamente $\tilde{\pi}$-invariante. Pela minimalidade de $A$ obtemos

$$
\widetilde{L}^{+}(x)=A \text {. }
$$

Mostraremos, agora, a condição suficiente. Suponhamos que $A$ não seja minimal, ou seja, existe um subconjunto próprio de $A, B \varsubsetneqq A$, tal que $B \backslash M \neq \emptyset, B$ é fechado e $B \backslash M$ é positivamente $\tilde{\pi}$-invariante. Dado $b \in B \backslash M$, segue que $b \in A \backslash M$. Daí por hipótese temos $A=\widetilde{L}^{+}(b)$. Como $B \backslash M$ é positivamente $\widetilde{\pi}$-invariante e $B$ é fechado, obtemos

$$
B \subset A=\widetilde{L}^{+}(b) \subset B
$$

Portanto $A=B$ e isto é uma contradição. Assim $A$ não admite subconjunto próprio e $A$ é minimal.

Pela prova do Teorema 2.1.4, temos o seguinte resultado.

Teorema 2.1.5 [13, Teorema 4.3] Sejam $A \subset X$ um conjunto minimal em $(X, \pi ; M, I)$ e $x \in A \backslash M$ um ponto tal que $\widetilde{L}^{+}(x) \backslash M \neq \emptyset$. Então $A=\widetilde{L}^{+}(x)$.

O próximo lema mostra que a órbita positiva de um ponto $x \in X$ é contínua sempre que $\tilde{\pi}^{+}(x)$ é minimal. 
Lema 2.1.6 [13, Lema 4.1] Seja $(X, \pi ; M, I)$ um sistema semidinâmico impulsivo. Se $\tilde{\pi}^{+}(x)$ é minimal então $\tilde{\pi}^{+}(x)=\pi^{+}(x)$.

Demonstração: Para provar que $\tilde{\pi}^{+}(x)=\pi^{+}(x)$ é suficiente mostrarmos que $\phi(x)=+\infty$. Suponhamos que $\phi(x)<+\infty$. Então $x_{1}=\pi(x, \phi(x)) \in M$, e $x_{1} \in \overline{\widetilde{\pi}^{+}(x)}$. Como $\tilde{\pi}^{+}(x)$ é fechado, pois é minimal, segue que $x_{1} \in \tilde{\pi}^{+}(x)$ o que é uma contradição, pois, pela descrição da trajetória impulsiva, pontos de impulsos não podem estar na trajetória de $x$. Portanto $\phi(x)=+\infty$.

Na teoria de sistemas dinâmicos contínuos, é sabido que todo conjunto não vazio, compacto e invariante contém um subconjunto minimal compacto, veja [3, Teorema 4.4 (Capítulo III)]. Nas próximas linhas apresentamos condições para obter este resultado no caso de sistemas semidinâmicos com impulsos. Para isso, definimos inicialmente um tipo especial de minimalidade em $(X, \pi ; M, I)$

Definição 2.1.7 Dizemos que um conjunto $A \subset X$ é $I$-minimal em $(X, \pi ; M, I)$ se as seguintes condições forem satisfeitas:

i) $A \backslash M \neq \emptyset$;

ii) A é fechado;

iii) $A \backslash M$ é positivamente $\tilde{\pi}$ - invariante;

iv) $I(A \cap M) \subset A$;

v) $A$ não admite subconjunto próprio satisfazendo as propriedades $i$ ), ii), iii) e $i v$ ).

Supondo que exista $\varepsilon_{0}>0$ tal que $\pi\left(M,\left[0, \varepsilon_{0}\right]\right) \cap I(M)=\emptyset$, podemos dar uma caracterização para os conjuntos I-minimais, veja Teorema 2.1.9 abaixo. Para isso, apresentamos um resultado auxiliar que caracteriza a $I$-invariância do conjunto $\widetilde{L}^{+}(x), x \in X$.

Lema 2.1.8 [13, Lema 4.2] Seja $(X, \pi ; M, I)$ um sistema semidinâmico impulsivo e suponha que exista $\varepsilon_{0}>0$ tal que $\pi\left(M,\left[0, \varepsilon_{0}\right]\right) \cap I(M)=\emptyset$. Então $I\left(\widetilde{L}^{+}(x) \cap M\right) \subset \widetilde{L}^{+}(x), x \in X$. 
Demonstração: Suponhamos que $\widetilde{L}^{+}(x) \cap M \neq \emptyset$ para algum $x \in X$. Seja $a \in \widetilde{L}^{+}(x) \cap M$. Então existe uma sequência $\left\{t_{n}\right\}_{n \geq 1} \subset \mathbb{R}_{+}$tal que $t_{n} \stackrel{n \rightarrow+\infty}{\longrightarrow}+\infty \mathrm{e}$

$$
\tilde{\pi}\left(x, t_{n}\right) \stackrel{n \rightarrow+\infty}{\longrightarrow} a
$$

Como $M$ satisfaz a condição STC, existe um STC-tubo $F(L,[0,2 \lambda])$ através de $a$ dado por uma seção $S \subset M$ tal que $S=M \cap F(L,[0,2 \lambda])$. Podemos assumir $\lambda<\varepsilon_{0}$. Além disso, como o tubo é uma vizinhança de $a$, existe um $\eta>0$ tal que

$$
B(a, \eta) \subset F(L,[0,2 \lambda])
$$

Consideremos $H_{1}=F(L,(\lambda, 2 \lambda]) \cap B(a, \eta)$ e $H_{2}=F(L,[0, \lambda]) \cap B(a, \eta)$ como mostra a Figura 2.1.

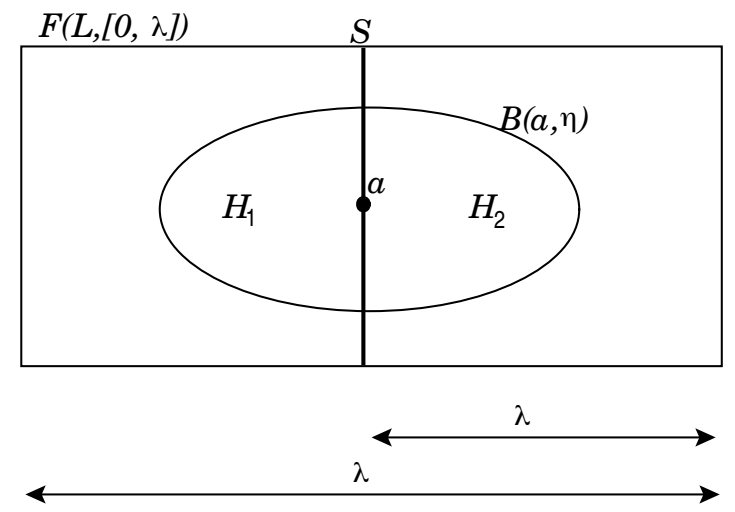

Figura 2.1: Conjuntos $H_{1}$ e $H_{2}$.

Seja $\left\{\tilde{\pi}\left(x, t_{n_{k}}\right)\right\}_{k \geq 1}$ uma subsequência qualquer de $\left\{\tilde{\pi}\left(x, t_{n}\right)\right\}_{n \geq 1}$. Afirmamos que existe um número natural $\ell>0$ tal que $\left\{\tilde{\pi}\left(x, t_{n_{k}}\right)\right\}_{n_{k} \geq \ell} \subset H_{1}$. De fato, suponhamos o contrário, e por comodidade assumimos, que $\left\{\tilde{\pi}\left(x, t_{n_{k}}\right)\right\}_{k \geq 1} \subset H_{2}$. Seja $y_{k}=\tilde{\pi}\left(x, t_{n_{k}}\right), k=1,2, \ldots$. Pela propriedade de tubo, existe $s_{k} \in[0, \lambda]$ tal que $F\left(y_{k}, s_{k}\right) \subset S$ para cada $k=1,2, \ldots$, isto é,

$$
\pi\left(F\left(y_{k}, s_{k}\right), s_{k}\right)=y_{k},
$$

$k=1,2, \ldots$. Seja $k_{0}>0$ tal que $t_{n_{k}}-s_{k}>0$ para todo $k \geq k_{0}$. Como $\tilde{\pi}(x, t) \notin \bigcup_{n=1}^{+\infty} F\left(y_{n}, s_{n}\right)$ para 
todo $t>0$, pois $I(M) \cap M=\emptyset$ (Hipótese $(\mathrm{H} 2))$, existem $t_{k}^{\prime} \in\left(t_{n_{k}}-s_{k}, t_{n_{k}}\right]$ e $w_{k} \in M$ tais que

$$
I\left(w_{k}\right)=\tilde{\pi}\left(x, t_{k}^{\prime}\right) \quad \text { para todo } k \geq k_{0} .
$$

Também, notemos que

$$
I\left(w_{k}\right)=\tilde{\pi}\left(x, t_{k}^{\prime}\right) \in F(L,[0, \lambda]) \quad \text { para todo } k \geq k_{0} .
$$

Como $F(L,[0, \lambda]) \subset \pi\left(M,\left[0, \varepsilon_{0}\right]\right)$, temos

$$
I\left(w_{k}\right) \in \pi\left(M,\left[0, \varepsilon_{0}\right]\right) \cap I(M),
$$

para todo $k \geq k_{0}$, o que contradiz a hipótese. Desta forma, existe $\ell>0$ tal que $\left\{\tilde{\pi}\left(x, t_{n_{k}}\right)\right\}_{n_{k} \geq \ell} \subset H_{1}$. $\operatorname{Logo} \mu_{n_{k}}=\phi\left(\tilde{\pi}\left(x, t_{n_{k}}\right)\right) \stackrel{k \rightarrow+\infty}{\longrightarrow} 0 \mathrm{e}$

$$
I\left(\pi\left(\tilde{\pi}\left(x, t_{n_{k}}\right), \mu_{n_{k}}\right)\right) \stackrel{k \rightarrow+\infty}{\longrightarrow} I(a),
$$

ou seja,

$$
\tilde{\pi}\left(x, t_{n_{k}}+\mu_{n_{k}}\right) \stackrel{k \rightarrow+\infty}{\longrightarrow} I(a)
$$

Portanto, $I(a) \in \widetilde{L}^{+}(x)$ e o lema está provado.

Teorema 2.1.9 [13, Teorema 4.4] Seja $(X, \pi ; M, I)$ um sistema semidinâmico impulsivo e suponha que exista $\varepsilon_{0}>0$ tal que $\pi\left(M,\left[0, \varepsilon_{0}\right]\right) \cap I(M)=\emptyset$. Então o conjunto $A \subset X$ é I-minimal se, $e$ somente se, $A=\overline{\widetilde{\pi}^{+}(x)}$ para todo $x \in A \backslash M$.

Demonstração: É suficiente usar o Lema 2.1.8 e a prova do Teorema 2.1.3.

Corolário 2.1.10 [13, Corolário 4.1] Seja $(X, \pi ; M, I)$ um sistema semidinâmico impulsivo e suponha que exista $\varepsilon_{0}>0$ tal que $\pi\left(M,\left[0, \varepsilon_{0}\right]\right) \cap I(M)=\emptyset$. Então A é I-minimal se, e somente se, $A$ é minimal.

O resultado a seguir apresenta condições para um conjunto compacto conter um subconjunto minimal. 
Teorema 2.1.11 [13, Teorema 4.5] Seja $(X, \pi ; M, I)$ um sistema semidinâmico impulsivo e suponha que exista $\varepsilon_{0}>0$ tal que $\pi\left(M,\left[0, \varepsilon_{0}\right]\right) \cap I(M)=\emptyset$. Seja $F \subset X$ um conjunto compacto tal que $F \backslash M$ é não vazio e positivamente $\tilde{\pi}$-invariante e $F$ é $I$-invariante. Então $F$ contém um subconjunto minimal.

Demonstração: Mostraremos que $F$ contém um subconjunto $I$-minimal utilizando o Lema de Zorn. Consideremos a familia

$\sigma=\{B \subset F: B \backslash M \neq \emptyset, B$ é compacto, $B \backslash M$ é positivamente $\tilde{\pi}$-invariante e $B$ é $I$-invariante $\}$.

É claro que $\sigma \neq \emptyset$ pois $F \in \sigma$. Dados $B_{1}, B_{2} \in \sigma$, definimos a relação de ordem parcial da forma seguinte:

$$
B_{1} \leq B_{2} \text { se, e somente se, } B_{1} \subseteq B_{2} \text {. }
$$

Seja $\sigma^{*}=\left\{B_{\lambda}\right\}_{\lambda \in \Lambda}$ um subconjunto totalmente ordenado de $\sigma$ pela relação $\leq$. Observe que se $B_{\lambda_{1}}, B_{\lambda_{2}}, \ldots, B_{\lambda_{n}} \in \sigma^{*}$, então

$$
\bigcap_{i=1}^{n} B_{\lambda_{i}}=\text { o menor } \operatorname{dos} B_{\lambda_{i}}{ }^{\prime} s
$$

e como cada $B_{\lambda_{i}} \neq \emptyset$, pois $B_{\lambda_{i}} \backslash M \subset B_{\lambda_{i}}$ e $B_{\lambda_{i}} \backslash M \neq \emptyset$, temos

$$
\bigcap_{i=1}^{n} B_{\lambda_{i}} \neq \emptyset
$$

Seja $J=\bigcap_{\lambda \in \Lambda} B_{\lambda}$. Afirmamos que $J \in \sigma$. De fato, note que:

- $J \neq \emptyset$. De fato, suponhamos por absurdo que $J=\emptyset$, então $F \subset \bigcup_{\lambda \in \Lambda} B_{\lambda}^{c}$, ou seja, a família $\left\{B_{\lambda}^{c}\right\}_{\lambda \in \Lambda}$ é uma cobertura aberta de $F$ e como $F$ é compacto, existem $\lambda_{1}, \lambda_{2}, \ldots, \lambda_{n} \in \Lambda$ tais que

$$
F \subset \bigcup_{i=1}^{n} B_{\lambda_{i}}^{c}
$$

Ou seja,

$$
\bigcap_{i=1}^{n} B_{\lambda_{i}} \subset F^{c} .
$$

Como $B_{\lambda_{i}} \subset F$ (ou equivalentemente $F^{c} \subset B_{\lambda_{i}}^{c}$ ) para todo $i=1,2, \ldots, n$, segue que $\bigcap_{i=1}^{n} B_{\lambda_{i}} \subset$ $B_{\lambda_{i}}^{c}$ para todo $i=1,2, \ldots, n$. Então 


$$
\bigcap_{i=1}^{n} B_{\lambda_{i}}=\emptyset
$$

o que contradiz (2.1). Portanto $J \neq \emptyset$.

- $J \subset F$, pois $B_{\lambda} \subset F$ para todo $\lambda \in \Lambda$.

- $J$ é compacto, pois está contido em $F$ que é compacto.

- $J \backslash M \neq \emptyset$. Suponhamos que $J \backslash M=\emptyset$, então $J=\bigcap_{\lambda \in \Lambda} B_{\lambda} \subset M$. Seja $x \in J$, então $x \in B_{\lambda} \cap M$ para todo $\lambda \in \Lambda$. Como cada $B_{\lambda}$ é $I$-invariante, temos que

$$
I(x) \in B_{\lambda} \backslash M
$$

para todo $\lambda \in \Lambda$, ou seja, $I(x) \in J \backslash M$ (lembremos que $I(M) \cap M=\emptyset$ ) o que é uma contradição. Portanto, $J \backslash M \neq \emptyset$.

- $J \backslash M$ é positivamente $\tilde{\pi}$ - invariante. De fato, seja $x \in J \backslash M=\bigcap_{\lambda \in \Lambda}\left(B_{\lambda} \backslash M\right)$, então $x \in B_{\lambda} \backslash M$ para todo $\lambda \in \Lambda$ e como cada $B_{\lambda} \backslash M$ é positivamente $\widetilde{\pi}$-invariante temos

$$
\tilde{\pi}(x, t) \in B_{\lambda} \backslash M, \text { para todo } t \geq 0 \text { e para todo } \lambda \in \Lambda \text {. }
$$

Logo

$$
\tilde{\pi}(x, t) \in \bigcap_{\lambda \in \Lambda}\left(B_{\lambda} \backslash M\right)=J \backslash M, \text { para todo } t \geq 0 .
$$

Como $x \in J \backslash M$ é arbitrário segue que $J \backslash M$ é positivamente $\tilde{\pi}$-invariante.

- $J$ é $I$-invariante. Seja $x \in J \cap M$, então $x \in B_{\lambda} \cap M$ para todo $\lambda \in \Lambda$. Como cada $B_{\lambda}$ é I-invariante temos

$$
I(x) \in B_{\lambda}
$$

para todo $\lambda \in \Lambda$, isto é, $I(x) \in J$.

Assim podemos concluir que $J=\bigcap_{\lambda \in \Lambda} B_{\lambda} \in \sigma$.

Por outro lado, se $B \in \sigma^{*}$ segue que $J \subseteq B$. Logo pelo Lema de Zorn segue que $\sigma$ contém um 
elemento minimal que é o subconjunto $I$-minimal de $F$. Pelo Corolário 2.1.10 este subconjunto I-minimal é minimal.

Temos também o seguinte resultado.

Teorema 2.1.12 [13, Teorema 4.6] Seja $(X, \pi ; M, I)$ um sistema semidinâmico impulsivo e suponha que exista $\varepsilon_{0}>0$ tal que $\pi\left(M,\left[0, \varepsilon_{0}\right]\right) \cap I(M)=\emptyset$. Seja $x \in X$ tal que $\overline{\widetilde{\pi}^{+}(x)}$ é compacto $e$ $\widetilde{L}^{+}(x) \backslash M \neq \emptyset$. Então $\widetilde{L}^{+}(x)$ contém um subconjunto minimal compacto.

Demonstração: Como $\overline{\widetilde{\pi}^{+}(x)}$ é compacto, segue que $\widetilde{L}^{+}(x)$ é não vazio e compacto. Pela hipótese temos $\widetilde{L}^{+}(x) \backslash M \neq \emptyset$. Como $\widetilde{L}^{+}(x) \backslash M$ é positivamente $\widetilde{\pi}$-invariante (Teorema 1.4.7 ) e $\widetilde{L}^{+}(x)$ é I-invariante (Lema 2.1.8), segue do Teorema 2.1.11 que $\widetilde{L}^{+}(x)$ contém um subconjunto minimal. 
Capítulo

3

\section{Movimentos recorrentes e quase periódicos}

Neste capítulo, definimos os conceitos de movimentos recorrentes e quase periódicos em sistemas semidinâmicos impulsivos. Além disso, apresentamos alguns resultados que relacionam os conceitos de minimalidade, movimentos recorrentes e movimentos quase periódicos. Alguns resultados da teoria clássica de sistemas dinâmicos são generalizados para o caso impulsivo, como por exemplo o Teorema de Birkhoff. Os resultados deste capítulo estão apresentados no artigo [13].

\subsection{Recorrência}

Definição 3.1.1 Um ponto $x \in X$ é chamado de $\tilde{\pi}$-recorrente se, dado $\varepsilon>0$, existe $T=T(\varepsilon)>0$ tal que para todos $t, s \geq 0$, o intervalo $[0, T]$ contém um número $\tau>0$ tal que

$$
d(\tilde{\pi}(x, t), \tilde{\pi}(x, s+\tau))<\varepsilon .
$$

Uma órbita positiva $\tilde{\pi}^{+}(x)$ é chamada de $\tilde{\pi}$-recorrente se $x \in X$ é $\tilde{\pi}$-recorrente.

Observação 3.1.2 Se $x \in X$ é $\tilde{\pi}$-recorrente, então dado $\varepsilon>0$ existe $T=T(\varepsilon)>0$ tal que $\tilde{\pi}^{+}(x) \subset$ $B(\tilde{\pi}(x,[t, t+T]), \varepsilon)$, para todo $t \geq 0$.

O resultado seguinte apresenta condições suficientes para um ponto ser $\widetilde{\pi}$-recorrente. 
Teorema 3.1.3 [13, Teorema 4.7] Sejam $(X, \pi ; M, I)$ um sistema semidinâmico impulsivo e $A \subset X$ um subconjunto compacto e minimal. Se $x \in A \backslash M$, então $x$ é $\tilde{\pi}$-recorrente.

Demonstração: Suponhamos que $x$ não seja $\tilde{\pi}$-recorrente, isto é, existem $\varepsilon>0$ e sequências $\left\{T_{n}\right\}_{n \geq 1},\left\{s_{n}\right\}_{n \geq 1},\left\{t_{n}\right\}_{n \geq 1} \subset \mathbb{R}_{+}$tais que $T_{n} \stackrel{n \rightarrow+\infty}{\longrightarrow}+\infty \mathrm{e}$

$$
d\left(\tilde{\pi}\left(x, t_{n}\right), \tilde{\pi}\left(x, s_{n}+\tau\right)\right) \geq \varepsilon, \text { para todo } \tau \in\left[0, T_{n}\right], \quad n=1,2, \ldots
$$

Como $x \in A \backslash M$ e $A \backslash M$ é positivamente $\widetilde{\pi}$-invariante, temos

$$
\left\{\tilde{\pi}\left(x, t_{n}\right)\right\}_{n \geq 1} \subset A \backslash M \subset A \quad \text { e } \quad\left\{\tilde{\pi}\left(x, s_{n}+\frac{T_{n}}{2}\right)\right\}_{n \geq 1} \subset A \backslash M \subset A .
$$

Como $A$ é compacto, podemos supor, passando para subsequência se for preciso, que

$$
\begin{gathered}
\tilde{\pi}\left(x, t_{n}\right) \quad \stackrel{n \rightarrow+\infty}{\longrightarrow} a \in A, \\
\tilde{\pi}\left(x, s_{n}+\frac{T_{n}}{2}\right) \stackrel{n \rightarrow+\infty}{\longrightarrow} b \in A .
\end{gathered}
$$

Caso 1: $b \notin M$. Seja $t \geq 0$ arbitrário e fixado. Primeiro suponhamos que $t \neq \sum_{j=0}^{k} \phi\left(b_{j}^{+}\right)$para todo $k=0,1,2, \ldots$. Neste caso, pela continuidade de $\pi$ e $I$, existe $\delta>0$ tal que se $d(y, b)<\delta$ então

$$
d(\tilde{\pi}(y, t), \tilde{\pi}(b, t))<\frac{\varepsilon}{3} .
$$

Por outro lado, podemos obter $n_{0} \geq 1$ tal que

$$
\begin{array}{rlrl}
\frac{T_{n_{0}}}{2} & >t, & & \\
d\left(\tilde{\pi}\left(x, t_{n}\right), a\right) & <\frac{\varepsilon}{3}, \quad & \text { para todo } n \geq n_{0}, \\
d\left(\tilde{\pi}\left(x, s_{n}+\frac{T_{n}}{2}\right), b\right) & <\delta, \quad \text { para todo } n \geq n_{0} .
\end{array}
$$

Usando (3.1), (3.2) e (3.3), obtemos

$$
\begin{gathered}
d(\tilde{\pi}(b, t), a) \geq d\left(\tilde{\pi}\left(x, s_{n_{0}}+\frac{T_{n_{0}}}{2}+t\right), a\right)-d\left(\tilde{\pi}\left(x, s_{n_{0}}+\frac{T_{n_{0}}}{2}+t\right), \tilde{\pi}(b, t)\right) \\
\geq d\left(\tilde{\pi}\left(x, s_{n_{0}}+\frac{T_{n_{0}}}{2}+t\right), \tilde{\pi}\left(x, t_{n_{0}}\right)\right)-d\left(a, \tilde{\pi}\left(x, t_{n_{0}}\right)\right)-d\left(\widetilde{\pi}\left(x, s_{n_{0}}+\frac{T_{n_{0}}}{2}+t\right), \tilde{\pi}(b, t)\right)
\end{gathered}
$$




$$
>\varepsilon-\frac{\varepsilon}{3}-\frac{\varepsilon}{3}=\frac{\varepsilon}{3} \text {. }
$$

Como a escolha de $t$ é arbitrária, podemos concluir que

$$
d(\tilde{\pi}(b, t), a) \geq \frac{\varepsilon}{3} \quad \text { para todo } t \geq 0 \text { tal que } t \neq \sum_{j=0}^{k} \phi\left(b_{j}^{+}\right), k=0,1,2, \ldots
$$

Agora, suponhamos que exista $k=0,1,2, \ldots$, tal que $t=\sum_{j=0}^{k} \phi\left(b_{j}^{+}\right)$. Podemos tomar uma sequência $\left\{\lambda_{n}\right\}_{n \geq 1}$ de números reais positivos tal que

$$
\lambda_{n} \stackrel{n \rightarrow+\infty}{\longrightarrow} \sum_{j=0}^{k} \phi\left(b_{j}^{+}\right) \operatorname{com} \sum_{j=0}^{k} \phi\left(b_{j}^{+}\right)<\lambda_{n}<\sum_{j=0}^{k+1} \phi\left(b_{j}^{+}\right), \quad k=0,1,2, \ldots
$$

Usando (3.4), podemos escrever

$$
d\left(\tilde{\pi}\left(b, \lambda_{n}\right), a\right) \geq \frac{\varepsilon}{3},
$$

para todo $n \geq 1$. Pela continuidade à direita de $\tilde{\pi}$ obtemos

$$
d\left(\tilde{\pi}\left(b, \sum_{j=0}^{k} \phi\left(b_{j}^{+}\right)\right), a\right) \geq \frac{\varepsilon}{3}
$$

Logo podemos concluir que

$$
d(\widetilde{\pi}(b, t), a) \geq \frac{\varepsilon}{3} \quad \text { para todo } t \geq 0,
$$

e portanto

$$
a \notin \overline{\widetilde{\pi}^{+}(b)},
$$

o que é uma contradição, pois deveríamos ter $\overline{\widetilde{\pi}^{+}(b)}=A$ já que $A$ é minimal e $b \in A \backslash M$.

Caso 2: $b \in M$. Como $M$ satisfaz a condição STC, existe um STC-tubo $F(L,[0,2 \lambda])$ através de $b$ dado por uma seção $S$. Além disso, o tubo é uma vizinhança de $b$, logo existe um $\eta>0$ tal que

$$
B(b, \eta) \subset F(L,[0,2 \lambda]) .
$$


Chamemos de $H_{1}=F(L,(\lambda, 2 \lambda]) \cap B(b, \eta)$ e $H_{2}=F(L,[0, \lambda]) \cap B(b, \eta)$. Seja $w_{n}=\tilde{\pi}\left(x, s_{n}+\frac{T_{n}}{2}\right)$, $n=1,2,3, \ldots$ e observe que $w_{n} \stackrel{n \rightarrow+\infty}{\longrightarrow} b$. Aqui, precisamos analisar dois casos: quando $\left\{w_{n}\right\}_{n \geq 1}$ admite subsequência em $H_{1}$ e quando $\left\{w_{n}\right\}_{n \geq 1}$ admite subsequência em $H_{2}$.

- Suponhamos, primeiramente, que a sequência $\left\{w_{n}\right\}_{n \geq 1}$ possua uma subsequência $\left\{w_{n_{r}}\right\}_{r \geq 1}$ em $H_{1}$. Neste caso, temos $\phi\left(w_{n_{r}}\right) \stackrel{r \rightarrow+\infty}{\longrightarrow} 0 . \operatorname{Logo}$

$$
\tilde{\pi}\left(w_{n_{r}}, \phi\left(w_{n_{r}}\right)\right) \stackrel{r \rightarrow+\infty}{\longrightarrow} I(b),
$$

isto é,

$$
\tilde{\pi}\left(x, s_{n_{r}}+\frac{T_{n_{r}}}{2}+\phi\left(w_{n_{r}}\right)\right) \stackrel{r \rightarrow+\infty}{\longrightarrow} I(b) .
$$

Como $A \backslash M$ é positivamente $\tilde{\pi}$-invariante e $x \in A \backslash M$, segue que $I(b) \in \bar{A}=A$.Vamos considerar o semifluxo $\tilde{\pi}(I(b), t)$. Note que $I(b) \notin M$, pois $I(M) \cap M=\emptyset$. Se $t \neq \sum_{j=0}^{k} \phi\left(I(b)_{j}^{+}\right)$para todo $k=1,2, \ldots$, segue da continuidade de $\pi$ e $I$ que existe $\delta>0$, tal que se $d(y, I(b))<\delta$, então

$$
d(\widetilde{\pi}(y, t), \widetilde{\pi}(I(b), t))<\frac{\varepsilon}{3}
$$

Tomemos agora um número natural $r_{0} \geq 1$ tal que

$$
\begin{gathered}
\frac{T_{n_{r_{0}}}}{2}>t+\phi\left(w_{n_{r_{0}}}\right), \\
d\left(\tilde{\pi}\left(x, t_{n_{r}}\right), a\right)<\frac{\varepsilon}{3}, \quad \text { para todo } r \geq r_{0}, \\
d\left(\tilde{\pi}\left(x, s_{n_{r}}+\frac{T_{n_{r}}}{2}+\phi\left(w_{n_{r}}\right)\right), I(b)\right)<\delta, \quad \text { para todo } r \geq r_{0} .
\end{gathered}
$$

Usando (3.1), (3.5) e (3.6) obtemos

$$
\begin{gathered}
\frac{T_{n_{r_{0}}}}{2}+\phi\left(w_{n_{r_{0}}}\right)+t<\frac{T_{n_{r_{0}}}}{2}+\frac{T_{n_{r_{0}}}}{2}=T_{n_{r_{0}}} \\
d(\tilde{\pi}(I(b), t), a) \geq d\left(\tilde{\pi}\left(x, s_{n_{r_{0}}}+\frac{T_{n_{r_{0}}}}{2}+\phi\left(w_{n_{r_{0}}}\right)+t\right), a\right)-d\left(\tilde{\pi}\left(x, s_{n_{r_{0}}}+\frac{T_{n_{r_{0}}}}{2}+\phi\left(w_{n_{r_{0}}}\right)+t\right), \tilde{\pi}(I(b), t)\right) \\
\geq d\left(\tilde{\pi}\left(x, s_{n_{r_{0}}}+\frac{T_{n_{r_{0}}}}{2}+\phi\left(w_{n_{r_{0}}}\right)+t\right), \tilde{\pi}\left(x, t_{n_{r_{0}}}\right)\right)-d\left(a, \tilde{\pi}\left(x, t_{n_{r_{0}}}\right)\right) \\
-d\left(\tilde{\pi}\left(x, s_{n_{r_{0}}}+\frac{T_{n_{r_{0}}}}{2}+\phi\left(w_{n_{r_{0}}}\right)+t\right), \tilde{\pi}(I(b), t)\right)
\end{gathered}
$$




$$
\geq \varepsilon-\frac{\varepsilon}{3}-\frac{\varepsilon}{3}=\frac{\varepsilon}{3}
$$

Como $t$ é arbitrário temos então,

$$
d(\tilde{\pi}(I(b), t), a) \geq \frac{\varepsilon}{3}, \text { para todo } t \geq 0 \text { tal que } t \neq \sum_{j=0}^{k} \phi\left(I(b)_{j}^{+}\right), k=0,1,2, \ldots
$$

Se $t=\sum_{j=0}^{k} \phi\left(I(b)_{j}^{+}\right)$para algum $k=0,1,2, \ldots$, tomamos uma sequência $\left\{\lambda_{n}\right\}_{n \geq 1}$ de números reais positivos tal que

$$
\lambda_{n} \stackrel{n \rightarrow+\infty}{\longrightarrow} \sum_{j=0}^{k} \phi\left(I(b)_{j}^{+}\right), \quad \operatorname{com} \sum_{j=0}^{k} \phi\left(I(b)_{j}^{+}\right)<\lambda_{n}<\sum_{j=0}^{k+1} \phi\left(I(b)_{j}^{+}\right), \quad k=0,1,2, \ldots
$$

daí usando (3.7) temos

$$
d\left(\widetilde{\pi}\left(I(b), \lambda_{n}\right), a\right) \geq \frac{\varepsilon}{3}
$$

para todo $n \in \mathbb{N}$. Pela continuidade à direita de $\widetilde{\pi}$ segue que

$$
d\left(\tilde{\pi}\left(I(b), \sum_{j=0}^{k} \phi\left(I(b)_{j}^{+}\right)\right), a\right) \geq \frac{\varepsilon}{3}
$$

Resumindo temos

$$
d(\tilde{\pi}(I(b), t), a) \geq \frac{\varepsilon}{3}, \quad \text { para todo } t \geq 0,
$$

de onde concluímos que

$$
a \notin \overline{\widetilde{\pi}^{+}(I(b))},
$$

o que é uma contradição, pois deveríamos ter $\overline{\widetilde{\pi}^{+}(I(b))}=A$ já que $A$ é minimal e $I(b) \in A \backslash M$.

- Suponhamos agora que a sequência $\left\{w_{n}\right\}_{n \geq 1}$ possua uma subsequência $\left\{w_{n_{s}}\right\}_{s \geq 1}$ em $H_{2}$. Consideremos $0<\lambda<\phi(b)$. Como $w_{n_{s}} \stackrel{s \rightarrow+\infty}{\longrightarrow} b$, temos

$$
\tilde{\pi}\left(w_{n_{s}}, \lambda\right) \stackrel{s \rightarrow+\infty}{\longrightarrow} \tilde{\pi}(b, \lambda)
$$

ou seja,

$$
\tilde{\pi}\left(x, s_{n_{s}}+\frac{T_{n_{s}}}{2}+\lambda\right) \stackrel{s \rightarrow+\infty}{\longrightarrow} \tilde{\pi}(b, \lambda) .
$$


Como $x \in A \backslash M$ e $A \backslash M$ é positivamente $\tilde{\pi}$-invariante segue que

$$
\tilde{\pi}\left(x, s_{n_{s}}+\frac{T_{n_{s}}}{2}+\lambda\right) \in A \backslash M \subset A, \quad \text { para todo } s=1,2, \ldots
$$

Consequentemente

$$
b_{1}=\tilde{\pi}(b, \lambda) \in \bar{A}=A
$$

e além disso, $b_{1} \notin M$, pois $I(M) \cap M=\emptyset$. Considerando o semifluxo $\tilde{\pi}\left(b_{1}, t\right)$ e, usando ainda a prova do Caso 1 , obtemos

$$
d\left(\widetilde{\pi}\left(b_{1}, t\right), a\right) \geq \frac{\varepsilon}{3}, \quad \text { para todo } t \geq 0
$$

ou seja,

$$
a \notin \overline{\widetilde{\pi}^{+}\left(b_{1}\right)}
$$

o que é uma contradição, pois $b_{1} \in A \backslash M$ e $A$ é minimal.

Portanto $x \in A \backslash M$ é $\tilde{\pi}$-recorrente e o teorema está provado.

O teorema seguinte apresenta condições para que o fecho de uma órbita positiva seja minimal.

Teorema 3.1.4 [13, Teorema 4.8] Sejam $(X, \pi ; M, I)$ um sistema semidinâmico impulsivo onde X é um espaço métrico completo e $x \in X$. Se $\tilde{\pi}^{+}(x)$ é $\tilde{\pi}$-recorrente, então o fecho $\overline{\tilde{\pi}^{+}(x)}$ é um conjunto compacto. Além disso, se $x \notin M$ então $\overline{\widetilde{\pi}^{+}(x)}$ é minimal.

Demonstração: Mostremos que $\overline{\tilde{\pi}^{+}(x)}$ é compacto. Seja $\varepsilon>0$ dado. Como $\tilde{\pi}^{+}(x)$ é $\tilde{\pi}$-recorrente, existe $T=T(\varepsilon)>0$ tal que

$$
\tilde{\pi}^{+}(x) \subset B\left(\tilde{\pi}(x,[0, T]), \frac{\varepsilon}{2}\right)
$$

isto é,

$$
d(\tilde{\pi}(x, t), \widetilde{\pi}(x,[0, T]))<\frac{\varepsilon}{2} \quad \text { para todo } t \geq 0 .
$$

Seja $y \in \overline{\tilde{\pi}^{+}(x)}$, então existe uma sequência de pontos $\left\{y_{n}\right\}_{n \geq 1} \subset \tilde{\pi}^{+}(x)$ tal que

$$
\lim _{n \rightarrow \infty} y_{n}=y
$$


Notemos que $y_{n}=\tilde{\pi}\left(x, t_{n}\right)$, para alguma sequência $\left\{t_{n}\right\}_{n \geq 1} \subset \mathbb{R}_{+}$. Usando (3.8) temos

$$
d\left(y_{n}, \tilde{\pi}(x,[0, T])\right)<\frac{\varepsilon}{2}
$$

para todo $n=1,2, \ldots$. Fazendo $n \rightarrow+\infty$ na desigualdade anterior, obtemos

$$
d(y, \widetilde{\pi}(x,[0, T])) \leq \frac{\varepsilon}{2} .
$$

Por outro lado, sabemos que $\overline{\widetilde{\pi}(x,[0, T])}$ é compacto (veja [11, Lema 3.6]), logo ele é totalmente limitado, isto é, existem pontos $x_{1}, x_{2}, \ldots, x_{n} \in \overline{\widetilde{\pi}(x,[0, T])}$ tais que

$$
\overline{\widetilde{\pi}(x,[0, T])} \subset \bigcup_{i=1}^{n} B\left(x_{i}, \frac{\varepsilon}{2}\right) .
$$

Por compacidade, existe $z \in \overline{\widetilde{\pi}(x,[0, T])}$ tal que

$$
d(y, z)=d(y, \overline{\tilde{\pi}(x,[0, T])}) .
$$

Por (3.10) existe $x_{i} \in \overline{\widetilde{\pi}(x,[0, T])}$ com $i \in\{1,2, \ldots, n\}$ tal que

$$
d\left(z, x_{i}\right)<\frac{\varepsilon}{2}
$$

Assim usando (3.9), (3.11) e (3.12), obtemos

$$
d\left(y, x_{i}\right) \leq d(y, z)+d\left(z, x_{i}\right)<\frac{\varepsilon}{2}+\frac{\varepsilon}{2}=\varepsilon
$$

Portanto $y \in B\left(x_{i}, \varepsilon\right)$ e assim concluímos que

$$
\overline{\widetilde{\pi}^{+}(x)} \subset \bigcup_{i=1}^{n} B\left(x_{i}, \varepsilon\right),
$$

isto significa que $\overline{\widetilde{\pi}^{+}(x)}$ é totalmente limitado. Como $X$ é completo segue que $\overline{\widetilde{\pi}^{+}(x)}$ é compacto.

Vamos mostrar agora que $\overline{\widetilde{\pi}^{+}(x)}$ é minimal para $x \notin M$. Suponhamos o contrário, isto é, existe um conjunto $A \varsubsetneqq \overline{\tilde{\pi}^{+}(x)}$ tal que $A \backslash M \neq \emptyset, A$ é fechado e $A \backslash M$ é positivamente $\tilde{\pi}$-invariante. 
Note que $x \notin A$, pois $A \backslash M$ é positivamente $\widetilde{\pi}$-invariante e $A$ é fechado. Assim $d(x, A)=d>0$. Escolha $0<\varepsilon<\frac{d}{2}$. Segue da recorrência de $x$ que existe $T=T(\varepsilon)>0$ tal que para quaisquer $t, s \in \mathbb{R}_{+}$existe $\tau \in[0, T]$ tal que

$$
d(\tilde{\pi}(x, t), \tilde{\pi}(x, s+\tau))<\frac{\varepsilon}{2} .
$$

Tomemos $q \in A \backslash M$. Como $A$ é fechado e $A \backslash M$ é positivamente $\widetilde{\pi}$-invariante, segue que $\overline{\widetilde{\pi}^{+}(q)} \subset A$. Então

$$
d(x, \tilde{\pi}(q, t)) \geq d(x, A)=d>2 \varepsilon \text { para todo } t \geq 0 .
$$

Por outro lado, como $q \in \overline{\widetilde{\pi}^{+}(x)}$ e $q \notin M$, segue do Lema 1.4.11 que $q \in \widetilde{\pi}^{+}(x)$ ou $q \in \widetilde{L}^{+}(x)$.

Suponhamos, primeiramente, que $q \in \tilde{\pi}^{+}(x)$. Então $q=\tilde{\pi}(x, s)$ para algum $s>0$. Usando (3.13) obtemos

$$
d(x, \tilde{\pi}(x, s+t))>2 \varepsilon,
$$

para todo $t \geq 0$. Isto é uma contradição, pois $x$ é $\widetilde{\pi}$-recorrente.

Suponhamos agora que $q \in \widetilde{L}^{+}(x)$. Então existe uma sequência $\left\{\lambda_{n}\right\}_{n \geq 1} \subset \mathbb{R}_{+}$tal que $\lambda_{n} \stackrel{n \rightarrow+\infty}{\longrightarrow}+\infty$ e $\tilde{\pi}\left(x, \lambda_{n}\right) \stackrel{n \rightarrow+\infty}{\longrightarrow} q$. Pela $\tilde{\pi}$-recorrência de $x$, existe $r_{n} \in[0, T]$ tal que

$$
d\left(x, \tilde{\pi}\left(x, \lambda_{n}+r_{n}\right)\right)<\frac{\varepsilon}{2}
$$

para todo $n=1,2, \ldots$. Podemos assumir que $r_{n} \stackrel{n \rightarrow+\infty}{\longrightarrow} r \in[0, T]$.

Como $r_{n} \stackrel{n \rightarrow+\infty}{\longrightarrow} r, \tilde{\pi}\left(x, \lambda_{n}\right) \stackrel{n \rightarrow+\infty}{\longrightarrow} q$ e $q \notin M$, segue pela prova do Lema 3.6 (Caso 1) de [11] que

$$
\tilde{\pi}\left(x, \lambda_{n}+r_{n}\right) \stackrel{n \rightarrow+\infty}{\longrightarrow} \tilde{\pi}(q, r),
$$

se $r \neq \sum_{j=0}^{k} \phi\left(q_{j}^{+}\right)$para todo $k \in \mathbb{N}$. E, se $r=\sum_{j=0}^{k} \phi\left(q_{j}^{+}\right)$para algum $k \in \mathbb{N}$ então

$$
\tilde{\pi}\left(x, \lambda_{n}+r_{n}\right) \stackrel{n \rightarrow+\infty}{\longrightarrow} q_{k+1}^{+}=\tilde{\pi}(q, r) \quad \text { ou } \quad \tilde{\pi}\left(x, \lambda_{n}+r_{n}\right) \stackrel{n \rightarrow+\infty}{\longrightarrow} q_{k+1} .
$$


Fazendo $n \rightarrow+\infty$ em (3.14), temos

$$
d(x, \tilde{\pi}(q, r)) \leq \frac{\varepsilon}{2}
$$

$\mathrm{ou}$

$$
\left.d\left(x, q_{k+1}\right)\right) \leq \frac{\varepsilon}{2}
$$

Se (3.15) ocorrer, segue de (3.13) a seguinte desigualdade

$$
2 \varepsilon<d(x, \widetilde{\pi}(q, r)) \leq \frac{\varepsilon}{2}
$$

o que é uma contradição.

Por outro lado, se (3.16) ocorrer, tomamos uma sequência $\left\{s_{n}\right\}_{n \geq 1} \subset \mathbb{R}_{+}$tal que $0<s_{n}<\phi\left(q_{k}^{+}\right)$e $s_{n} \stackrel{n \rightarrow+\infty}{\longrightarrow} \phi\left(q_{k}^{+}\right)$. Então por (3.13) obtemos

$$
d\left(x, \tilde{\pi}\left(q, \sum_{j=-1}^{k-1} \phi\left(q_{j}^{+}\right)+s_{n}\right)\right)=d\left(x, \pi\left(q_{k}^{+}, s_{n}\right)\right)>2 \varepsilon
$$

onde $\phi\left(q_{-1}^{+}\right):=0$. Então

$$
d\left(x, q_{k+1}\right)=\lim _{n \rightarrow+\infty} d\left(x, \pi\left(q_{k}^{+}, s_{n}\right)\right) \geq 2 \varepsilon
$$

o que contradiz (3.16).

Portanto $\overline{\widetilde{\pi}^{+}(x)}$ é minimal.

A definição a seguir, diz respeito ao conceito de conjuntos relativamente densos. Este conceito pode ser encontrado em [3, Definição 3.11 (Capitulo III)].

Definição 3.1.5 Um conjunto $D \subset \mathbb{R}_{+}$é chamado relativamente denso se existe $L>0$ tal que

$$
D \cap(\alpha, \alpha+L) \neq \emptyset \quad \text { para todo } \quad \alpha \geq 0
$$

Apresentamos, agora, um resultado que relaciona o conceito de recorrência e conjuntos relativamente densos. 
Teorema 3.1.6 [13, Teorema 4.9] Seja $(X, \pi ; M, I)$ um sistema semidinâmico impulsivo tal que $\overline{\widetilde{\pi}^{+}(x)}$ é compacto para algum $x \in X \backslash M$. A órbita positiva $\tilde{\pi}^{+}(x)$ é $\tilde{\pi}$-recorrente se, e somente se, para cada $\varepsilon>0$ o conjunto $K_{\varepsilon}=\left\{t \in \mathbb{R}_{+}: d(x, \tilde{\pi}(x, t))<\varepsilon\right\}$ é relativamente denso.

Demonstração: Suponhamos que $\tilde{\pi}^{+}(x)$ seja $\tilde{\pi}$-recorrente. Dado $\varepsilon>0$ existe $T=T(\varepsilon)>$ 0 tal que $d(\tilde{\pi}(x, t), \tilde{\pi}(x,[\alpha, \alpha+T]))<\varepsilon$ para todo $t \geq 0$ e para todo $\alpha \geq 0$. Em particular, $d(x, \tilde{\pi}(x,[\alpha, \alpha+T]))<\varepsilon$, para todo $\alpha \geq 0$. Logo

$$
K_{\mathcal{\varepsilon}} \cap[\alpha, \alpha+T] \neq \emptyset, \quad \text { para todo } \varepsilon>0 .
$$

Portanto, $K_{\varepsilon}$ é relativamente denso e neste caso $L=T$.

Suponha agora que $K_{\varepsilon}$ seja relativamente denso para todo $\varepsilon>0$. Vamos provar que $\tilde{\pi}^{+}(x)$ é $\tilde{\pi}$-recorrente. Como $\overline{\widetilde{\pi}^{+}(x)}$ é compacto, segue do Teorema 3.1.3 que é suficiente mostrar que $\overline{\widetilde{\pi}^{+}(x)}$ é minimal.

Suponhamos que $\overline{\widetilde{\pi}^{+}(x)}$ não seja minimal, então existe um subconjunto próprio $A \nsubseteq \overline{\widetilde{\pi}^{+}(x)}$ tal que $A \backslash M \neq \emptyset, A$ é fechado e $A \backslash M$ é positivamente $\tilde{\pi}$-invariante.

Note que $x \notin A$, pois $A \backslash M$ é positivamente $\widetilde{\pi}$-invariante e $A$ é fechado. Assim $d(x, A)=d>0$. Escolha $0<\varepsilon<\frac{d}{2}$. Segue da hipótese que existe $T=T(\varepsilon)>0$ tal que

$$
K_{\mathcal{E}} \cap[\alpha, \alpha+T] \neq \emptyset, \quad \text { para todo } \alpha \geq 0 \text {. }
$$

Tomemos $q \in A \backslash M$. Como $A \backslash M$ é positivamente $\tilde{\pi}$-invariante, segue que $\tilde{\pi}^{+}(q) \subset A$. Então

$$
d(x, \tilde{\pi}(q, t)) \geq d(x, A)=d>2 \varepsilon, \text { para todo } t \geq 0 .
$$

Por outro lado, como $q \in \overline{\widetilde{\pi}^{+}(x)}$ e $q \notin M$, segue do Lema 1.4.11 que $q \in \widetilde{\pi}^{+}(x)$ ou $q \in \widetilde{L}^{+}(x)$.

Suponhamos que $q \in \widetilde{\pi}^{+}(x)$. Então $q=\tilde{\pi}(x, s)$ para algum $s>0$. Usando (3.18) obtemos

$$
d(x, \tilde{\pi}(x, s+t))>2 \varepsilon
$$

para todo $t \geq 0$. Assim

$$
K_{\mathcal{\varepsilon}} \cap[s, s+T]=\emptyset,
$$


que contradiz (3.17).

Suponhamos, agora, que $q \in \widetilde{L}^{+}(x)$. Então existe uma sequência $\left\{\lambda_{n}\right\}_{n \geq 1} \subset \mathbb{R}_{+}$tal que $\lambda_{n} \stackrel{n \rightarrow+\infty}{\longrightarrow}+\infty$ e $\tilde{\pi}\left(x, \lambda_{n}\right) \stackrel{n \rightarrow+\infty}{\longrightarrow} q$. Pela hipótese, para cada $\lambda_{n}$, existe $\eta_{n} \in[0, T]$ tal que

$$
d\left(x, \tilde{\pi}\left(x, \lambda_{n}+\eta_{n}\right)\right)<\varepsilon
$$

para todo $n=1,2,3, \ldots$. Podemos assumir, sem perda de generalidade, que $\eta_{n} \stackrel{n \rightarrow+\infty}{\longrightarrow} \eta \in[0, T]$. Como $\eta_{n} \stackrel{n \rightarrow+\infty}{\longrightarrow} \eta, \tilde{\pi}\left(x, \lambda_{n}\right) \stackrel{n \rightarrow+\infty}{\longrightarrow} q$ e $q \notin M$, segue pela prova do Lema 3.6 (Caso 1) de [11] que

$$
\tilde{\pi}\left(x, \lambda_{n}+\eta_{n}\right) \stackrel{n \rightarrow+\infty}{\longrightarrow} \tilde{\pi}(q, \eta)
$$

se $\eta \neq \sum_{j=0}^{k} \phi\left(q_{j}^{+}\right)$para todo $k \in \mathbb{N}$. E, se $\eta=\sum_{j=0}^{k} \phi\left(q_{j}^{+}\right)$para algum $k \in \mathbb{N}$ então

$$
\tilde{\pi}\left(x, \lambda_{n}+\eta_{n}\right) \stackrel{n \rightarrow+\infty}{\longrightarrow} q_{k+1}^{+}=\tilde{\pi}(q, \eta) \quad \text { ou } \quad \tilde{\pi}\left(x, \lambda_{n}+\eta_{n}\right) \stackrel{n \rightarrow+\infty}{\longrightarrow} q_{k+1} .
$$

Fazendo $n \rightarrow+\infty$ em (3.19), temos

$$
d(x, \tilde{\pi}(q, \eta)) \leq \varepsilon
$$

$\mathrm{ou}$

$$
\left.d\left(x, q_{k+1}\right)\right) \leq \varepsilon
$$

Se (3.20) ocorrer, segue de (3.18) a seguinte desigualdade

$$
2 \varepsilon<d(x, \tilde{\pi}(q, \eta)) \leq \varepsilon
$$

o que é uma contradição.

Entretanto, se (3.21) ocorrer, tomamos uma sequência $\left\{s_{n}\right\}_{n \geq 1} \subset \mathbb{R}_{+}$tal que $0<s_{n}<\phi\left(q_{k}^{+}\right)$e $s_{n} \stackrel{n \rightarrow+\infty}{\longrightarrow} \phi\left(q_{k}^{+}\right)$. Então por (3.18) obtemos

$$
d\left(x, \tilde{\pi}\left(q, \sum_{j=-1}^{k-1} \phi\left(q_{j}^{+}\right)+s_{n}\right)\right)=d\left(x, \pi\left(q_{k}^{+}, s_{n}\right)\right)>2 \varepsilon
$$

onde $\phi\left(q_{-1}^{+}\right):=0$. Isto implica que $d\left(x, q_{k+1}\right)=\lim _{n \rightarrow+\infty} d\left(x, \pi\left(q_{k}^{+}, s_{n}\right)\right) \geq 2 \varepsilon$, que contradiz (3.21). 
Portanto $\overline{\tilde{\pi}^{+}(x)}$ é minimal e, pelo Teorema 3.1.3, segue que $\widetilde{\pi}^{+}(x)$ é $\tilde{\pi}$-recorrente.

\subsection{Movimentos quase periódicos}

Definição 3.2.1 Um ponto $x \in X$ é chamado quase $\tilde{\pi}$-periódico se, dado $\varepsilon>0$, existe $T=T(\varepsilon)>$ 0 tal que para todo $\alpha \geq 0$, o intervalo $[\alpha, \alpha+T]$ contém um número $\tau=\tau(\alpha)>0$ tal que

$$
d(\tilde{\pi}(x, t), \tilde{\pi}(x, t+\tau))<\varepsilon \quad \text { para todo } t \geq 0 .
$$

Lema 3.2.2 [13, Lema 4.3] Seja $(X, \pi ; M, I)$ um sistema semidinâmico impulsivo. Se $x \in X$ é quase $\tilde{\pi}$-periódico, então todo ponto $y \in \tilde{\pi}^{+}(x)$ também é quase $\tilde{\pi}$-periódico.

Demonstração: Dado $\varepsilon>0$, como $x \in X$ é quase $\widetilde{\pi}$-periódico, existe $T=T(\varepsilon)>0$ tal que para todo $\alpha \geq 0$ o intervalo $[\alpha, \alpha+T]$ contém um número $\tau=\tau(\alpha)$ tal que

$$
d(\tilde{\pi}(x, t), \tilde{\pi}(x, t+\tau))<\varepsilon \quad \text { para todo } t \geq 0 .
$$

Seja $y \in \tilde{\pi}^{+}(x)$, então $y=\tilde{\pi}(x, s)$ para algum $s \geq 0$. Para cada $\alpha \geq 0$, consideramos o número $\tau>0$ escolhido acima e assim por (3.22) obtemos

$$
\begin{aligned}
d(\tilde{\pi}(y, t), \tilde{\pi}(y, t+\tau)) & =d(\tilde{\pi}(\tilde{\pi}(x, s), t), \tilde{\pi}(\tilde{\pi}(x, s), t+\tau)) \\
& =d(\tilde{\pi}(x, s+t), \tilde{\pi}(x, s+t+\tau)) \\
& <\varepsilon
\end{aligned}
$$

para todo $t \geq 0$. Portanto para todo $\alpha \geq 0$ o intervalo $[\alpha, \alpha+T]$ contém um número $\tau=\tau(\alpha)>0$ tal que

$$
d(\tilde{\pi}(y, t), \tilde{\pi}(y, t+\tau))<\varepsilon \quad \text { para todo } t \geq 0
$$

isto é, $y \in \widetilde{\pi}^{+}(x)$ é quase $\widetilde{\pi}$-periódico.

O próximo resultado apresenta condições suficientes para que um ponto quase $\tilde{\pi}$-periódico seja $\tilde{\pi}$-recorrente. 
Teorema 3.2.3 [13, Teorema 4.10] Seja $(X, \pi ; M, I)$ um sistema semidinâmico impulsivo e suponha que $\overline{\tilde{\pi}^{+}(x)}$ seja compacto para algum $x \in X \backslash M$. Se $x$ é quase $\tilde{\pi}$-periódico, então $x$ é $\tilde{\pi}$ recorrente.

Demonstração: Dado $\varepsilon>0$, existe $T=T(\varepsilon)>0$ tal que para todo $\alpha \geq 0$, o intervalo $[\alpha, \alpha+T]$ contém um número $\tau=\tau(\alpha)>0$ tal que

$$
d(\tilde{\pi}(x, t), \tilde{\pi}(x, t+\tau))<\varepsilon \quad \text { para todo } t \geq 0 .
$$

Seja $K_{\varepsilon}=\left\{s \in \mathbb{R}_{+}: d(x, \tilde{\pi}(x, s))<\varepsilon\right\}$. Logo por (3.23) temos

$$
K_{\varepsilon} \cap[\alpha, \alpha+T] \neq \emptyset \quad \text { para todo } \alpha \geq 0 .
$$

Como $\varepsilon>0$ é arbitrário, segue do Teorema 3.1.6 que $x$ é $\widetilde{\pi}$-recorrente.

Seja $(X, \pi ; M, I)$ um sistema semidinâmico impulsivo, temos a seguinte definição.

Definição 3.2.4 Um ponto $x \in X$ é chamado ponto crítico ou estacionário em relação a $\widetilde{\pi}$, se $\tilde{\pi}(x, t)=x$ para todo $t \geq 0$. Um ponto $x \in X$ é chamado periódico em relação a $\tilde{\pi}$, se $\tilde{\pi}(x, t)=x$ para algum $t>0$ e $x$ não é ponto crítico. Agora, um ponto $x \in X$ é eventualmente periódico em relação a $\tilde{\pi}$, se $\tilde{\pi}(x, t)$ é periódico para algum $t \geq 0$.

Consideremos o seguinte resultado auxiliar.

Teorema 3.2.5 [9, Teorema 3.3 e 3.4] Seja $(X, \pi ; M, I)$ um sistema semidinâmico impulsivo tal que $X$ é completo. Seja $x \in X \backslash M$.

a) se $\widetilde{\pi}^{+}(x)=\widetilde{L}^{+}(x)$, então x é eventualmente periódico;

b) se $x \in \widetilde{L}^{+}(x)$ e x não é eventualmente periódico, então $\overline{\widetilde{L}^{+}(x)-\widetilde{\pi}^{+}(x)}=\widetilde{L}^{+}(x)$.

Nos Teoremas 3.2.6 e 3.2.7, dado $x \in X$ vamos supor que $\phi\left(x_{k}^{+}\right)<+\infty$ para todo $k=0,1,2, \ldots$ Lembremos que a sequência $\left\{x_{k}\right\}_{k \geq 1}$ representa todos os pontos impulsivos, ou seja, $x_{k+1}=\pi\left(x_{k}^{+}, \phi\left(x_{k}^{+}\right)\right)$, $k=0,1,2, \ldots$. Estes teoremas tratam sobre a relação entre movimentos periódicos e eventualmente periódicos. Se $\phi\left(x_{k}^{+}\right)=+\infty$ para algum $k \in \mathbb{N}$, os resultados continuam válidos. 
Teorema 3.2.6 [13, Teorema 4.11] Seja $(X, \pi ; M, I)$ um sistema semidinâmico impulsivo. Suponha que $X$ é completo e $\widetilde{L}^{+}(x) \backslash M \neq \emptyset$ para algum $x \in X \backslash M$. Se $\widetilde{\pi}^{+}(x) \cup\left\{x_{k}\right\}_{k \geq 1}$ é minimal, então $x$ é eventualmente periódico.

Demonstração: Suponhamos por absurdo que $x \in X \backslash M$ não seja eventualmente periódico . Como $\tilde{\pi}^{+}(x) \cup\left\{x_{k}\right\}_{k \geq 1}$ é minimal e $\widetilde{L}^{+}(x) \backslash M \neq \emptyset$, segue do Teorema 2.1.5 que

$$
\tilde{\pi}^{+}(x) \cup\left\{x_{k}\right\}_{k \geq 1}=\widetilde{L}^{+}(x) .
$$

Assim, $x \in \widetilde{L}^{+}(x)$ e pelo item $\left.b\right)$ do Teorema 3.2.5 temos

$$
\overline{\widetilde{L}^{+}(x)-\widetilde{\pi}^{+}(x)}=\widetilde{L}^{+}(x) .
$$

Pela igualdade (3.24) vem que

$$
\overline{\left\{x_{k}\right\}_{k \geq 1}}=\widetilde{L}^{+}(x)
$$

o que é uma contradição, pois, $\widetilde{L}^{+}(x) \backslash M \neq \emptyset$. Portanto, $x$ é eventualmente periódico.

Teorema 3.2.7 [13, Teorema 4.12] Seja $(X, \pi ; M, I)$ um sistema semidinâmico impulsivo e seja $x \in X \backslash M$ tal que $x \in \widetilde{L}^{+}(x)$. Se x é eventualmente periódico, então x é periódico.

Demonstração: Como $x$ é eventualmente periódico, existe $t \geq 0$ tal que $\tilde{\pi}(x, t)$ é periódico, isto é, existe $T>0$ tal que

$$
\tilde{\pi}(x, t+T)=\tilde{\pi}(x, t) .
$$

Caso 1: $T \neq \sum_{j=0}^{k} \phi\left(x_{j}^{+}\right)$para todo $k \in \mathbb{N}$.

Por hipótese $x \in \widetilde{L}^{+}(x)$, então existe uma sequência $\left\{t_{n}\right\}_{n \geq 1} \subset \mathbb{R}_{+}, t_{n} \stackrel{n \rightarrow+\infty}{\longrightarrow}+\infty$ tal que

$$
\tilde{\pi}\left(x, t_{n}\right) \stackrel{n \rightarrow+\infty}{\longrightarrow} x
$$

Como $x \notin M$ e $T \neq \sum_{j=0}^{k} \phi\left(x_{j}^{+}\right)$, para todo $k \in \mathbb{N}$, segue do Lema 1.4.6 que

$$
\tilde{\pi}\left(x, t_{n}+T\right) \stackrel{n \rightarrow+\infty}{\longrightarrow} \tilde{\pi}(x, T) .
$$


Por outro lado, existe um número natural $n_{0} \geq 1$ tal que $t_{n} \geq t$ para todo $n \geq n_{0}$. Além disso, temos que

$$
\tilde{\pi}\left(x, t_{n}+T\right)=\tilde{\pi}\left(x, t_{n}\right) \quad \text { para todo } n \geq n_{0} .
$$

Fazendo $n \rightarrow+\infty$ em (3.29) e usando (3.27) e (3.28) obtemos $\tilde{\pi}(x, T)=x$, isto é, $x$ é periódico.

Caso 2: $T=\sum_{j=0}^{k} \phi\left(x_{j}^{+}\right)$para algum $k \in \mathbb{N}$.

Neste caso tome $\varepsilon>0$ tal que $\varepsilon<\min \left\{\phi(x), \phi\left(x_{k+1}^{+}\right)\right\}$e $T+\varepsilon<\sum_{j=0}^{k+1} \phi\left(x_{j}^{+}\right)$. Como $x \in \widetilde{L}^{+}(x)$, então existe uma sequência $\left\{t_{n}\right\}_{n \geq 1} \subset \mathbb{R}_{+}, t_{n} \stackrel{n \rightarrow+\infty}{\longrightarrow}+\infty$ tal que

$$
\tilde{\pi}\left(x, t_{n}\right) \stackrel{n \rightarrow+\infty}{\longrightarrow} x
$$

Como $x \notin M$ e $0<\varepsilon<\phi(x)$ segue do Lema 1.4.6 que

$$
\tilde{\pi}\left(x, t_{n}+\varepsilon\right) \stackrel{n \rightarrow+\infty}{\longrightarrow} \tilde{\pi}(x, \varepsilon)
$$

Além disso, como $\sum_{j=0}^{k} \phi\left(x_{j}^{+}\right)<T+\varepsilon<\sum_{j=0}^{k+1} \phi\left(x_{j}^{+}\right)$, segue ainda do Lema 1.4.6 que

$$
\tilde{\pi}\left(x, t_{n}+T+\varepsilon\right) \stackrel{n \rightarrow+\infty}{\longrightarrow} \tilde{\pi}(x, T+\varepsilon) .
$$

Por outro lado, existe um número natural $n_{0} \geq 1$ tal que $t_{n} \geq t$ para todo $n \geq n_{0}$. Isto implica que $t_{n}+\varepsilon \geq t$ para todo $n \geq n_{0}$. Como $\widetilde{\pi}(x, t)$ é periódico com período $T$ temos

$$
\tilde{\pi}\left(x, t_{n}+\varepsilon+T\right)=\tilde{\pi}\left(x, t_{n}+\varepsilon\right) \quad \text { para todo } n \geq n_{0} .
$$

Fazendo $n \rightarrow+\infty$ na igualdade acima e usando (3.30) e (3.31) obtemos

$$
\tilde{\pi}(x, T+\varepsilon)=\tilde{\pi}(x, \varepsilon) .
$$


Como $\tilde{\pi}$ é contínua à direita e $\varepsilon>0$ é arbitrário, obtemos

$$
\tilde{\pi}(x, T)=\lim _{\varepsilon \rightarrow 0^{+}} \tilde{\pi}(x, T+\varepsilon)=\lim _{\varepsilon \rightarrow 0^{+}} \tilde{\pi}(x, \varepsilon)=x .
$$

Isto é, $x$ é um ponto periódico. Portanto o teorema está provado.

De acordo com o Teorema 2.1.5, Teorema 3.2.6 e o Teorema 3.2.7, podemos obter o seguinte resultado.

Corolário 3.2.8 [13, Corolário 4.2] Seja $(X, \pi ; M, I)$ um sistema semidinâmico impulsivo. Suponha que $X$ é completo e seja $x \in X \backslash M$ tal que $\widetilde{L}^{+}(x) \backslash M \neq \emptyset$. Se $\tilde{\pi}^{+}(x) \cup\left\{x_{k}\right\}_{k \geq 1}$ é minimal, então $x$ é periódico. 


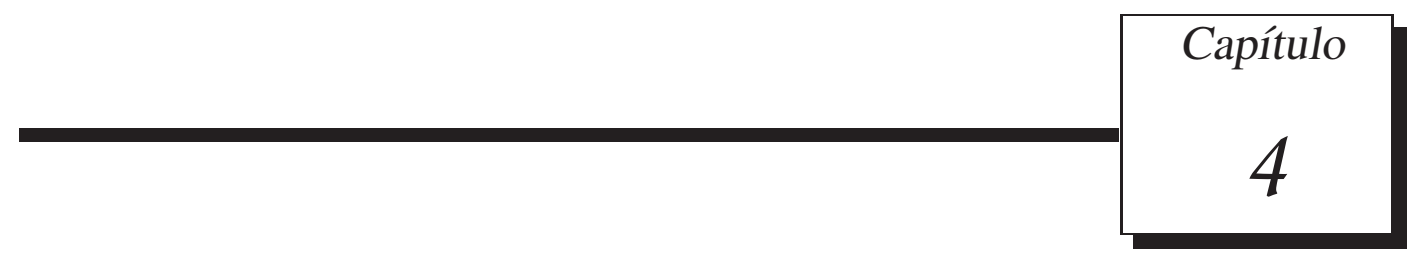

\section{Estabilidade de Lyapunov e de Zhukovskij}

Neste capítulo, apresentamos a teoria de estabilidade de Lyapunov e estabilidade de Zhukovskij para sistemas semidinâmicos com impulsos. Na seção 4.1, apresentamos o conceito de estabilidade de Lyapunov e estabelecemos alguns resultados que relacionam o conceito de estabilidade com os conceitos de recorrência, minimalidade e movimentos fracamente quase periódicos. Na seção 4.2 estudamos a teoria de estabilidade de Zhukovskij para sistemas impulsivos e obtemos alguns resultados sobre atratores uniformes. Os resultados deste capítulo estão presentes no artigo [14].

\subsection{Estabilidade de Lyapunov}

Seja $(X, \pi ; M, I)$ um sistema semidinâmico impulsivo. Dado $x \in X$, denotemos $\mathbb{N}(x)$ por

$$
\mathbb{N}(x)=\mathbb{N}=\{0,1,2,3, \ldots\} \quad \text { se } \quad x \in M
$$

$\mathrm{e}$

$$
\mathbb{N}(x)=\mathbb{N}^{*}=\{1,2,3, \ldots\} \quad \text { se } \quad x \notin M .
$$

Agora, dados $x \in X$ e $\varepsilon>0$, definimos o seguinte conjunto

$$
L(x, \varepsilon)=\left\{t \in \mathbb{R}_{+}:\left|t-t_{n}(x)\right|>\varepsilon, \text { para todo } n \in \mathbb{N}(x)\right\}
$$


onde $t_{n}(x)=\sum_{j=0}^{n-1} \phi\left(x_{j}^{+}\right)$para $n \geq 1, n \in \mathbb{N}$, e $t_{0}(x)=0$.

A seguir, apresentamos o conceito de ponto fracamente quase $\widetilde{\pi}$-periódico. Esta definição é dada por Kaul em [21].

Definição 4.1.1 Um ponto $x \in X$ é denominado fracamente quase $\tilde{\pi}$-periódico, se dado $\varepsilon>0$ existe $T=T(\varepsilon)>0$, tal que, para qualquer $\alpha \geq 0$, o intervalo $[\alpha, \alpha+T]$ contém um número $\tau=\tau(\alpha)>0$ tal que

$$
d(\tilde{\pi}(x, t), \tilde{\pi}(x, t+\tau))<\varepsilon \quad \text { para todo } \quad t \in L(x, \varepsilon) .
$$

O seguinte teorema apresenta condições para que um ponto fracamente quase $\widetilde{\pi}$-periódico seja $\tilde{\pi}$-recorrente.

Teorema 4.1.2 [14, Teorema 4.1] Sejam $(X, \pi ; M, I)$ um sistema semidinâmico impulsivo e $x \in$ $X \backslash M$ tal que $\overline{\tilde{\pi}^{+}(x)}$ é compacto. Se x é fracamente quase $\tilde{\pi}$-periódico, então xé $\tilde{\pi}$-recorrente.

Demonstração: Dado $\varepsilon>0$, podemos assumir que $0 \in L(x, \varepsilon)$ pois $x \in X \backslash M$. Por hipótese, existe $T=T(\varepsilon)>0$ tal que para todo $\alpha \geq 0$, o intervalo $[\alpha, \alpha+T]$ contém um número $\tau=\tau(\alpha)>0$ tal que

$$
d(\tilde{\pi}(x, t), \tilde{\pi}(x, t+\tau))<\varepsilon \quad \text { para todo } \quad t \in L(x, \varepsilon) .
$$

Seja $K_{\varepsilon}=\left\{s \in \mathbb{R}_{+}: d(x, \widetilde{\pi}(x, s))<\varepsilon\right\}$. Logo, por (4.1), temos que $K_{\mathcal{E}} \cap[\alpha, \alpha+T] \neq \emptyset$ para todo $\alpha \geq 0$, já que $0 \in L(x, \varepsilon)$. Assim, pelo Teorema 3.1.6, $x$ é $\widetilde{\pi}$-recorrente.

Se $x \in X \backslash M$, podemos escolher $\varepsilon_{0}>0$, suficientemente pequeno, tal que $0 \in L(x, \varepsilon)$ para todo $\varepsilon \in\left(0, \varepsilon_{0}\right)$. Assim, se $x$ é fracamente quase $\tilde{\pi}$-periódico, podemos obter uma sequência $\left\{t_{n}\right\}_{n \in \mathbb{N}} \subset \mathbb{R}_{+}, t_{n} \stackrel{n \rightarrow+\infty}{\longrightarrow}+\infty$, tal que $\pi\left(x, t_{n}\right) \stackrel{n \rightarrow+\infty}{\longrightarrow} x$. Portanto, temos o seguinte resultado.

Teorema 4.1.3 [14, Teorema 4.2] Sejam $(X, \pi ; M, I)$ um sistema semidinâmico impulsivo $e$ $x \in X \backslash M$. Se $x$ é fracamente quase $\tilde{\pi}$-periódico, então $x \in \widetilde{L}^{+}(x)$.

No caso contínuo, a recíproca do Teorema 4.1.3 não é verdade em geral. Isso também acontece 
no caso impulsivo, veja o Exemplo 4.1.5 a seguir. Antes de apresentarmos o exemplo, recordamos dois conceitos auxiliares. Veja a observação seguinte.

Observação 4.1.4 Seja $(X, \sigma)$ um sistema dinâmico discreto, veja [25] por exemplo. Um ponto $x \in X$ é chamado um ponto recorrente de $\sigma$ se dado $\varepsilon>0$, qualquer que seja $N \in \mathbb{N}$, existe um número natural $n>N$ tal que $\sigma^{n}(x) \in B(x, \varepsilon)$. Um ponto $x \in X$ é chamado um ponto quase periódico de $\sigma$ se dado $\varepsilon>0$, existe $N \in \mathbb{N}$ tal que $\left\{\sigma^{n+i}(x): i=0,1, \ldots, N\right\} \cap B(x, \varepsilon) \neq \emptyset$ para todo $n \in \mathbb{N}$.

Exemplo 4.1.5 [14, Exemplo 4.1] Seja $\left(Y, d_{Y}\right)$ um espaço métrico completo e considere um sistema dinâmico discreto $(Y, \sigma)$ com $\sigma$ contínua. Suponha que todo ponto de $Y$ é recorrente mas não é quase periódico. O leitor pode encontrar um exemplo deste tipo em [25, Exemplo 3.3]. Agora, defina o espaço $X=\mathbb{R} \times Y$ dotado com a métrica $d((t, x),(s, y))=\max \left\{|t-s|, d_{Y}(x, y)\right\}, t, s \in \mathbb{R}$ e $x, y \in Y$. Consideremos o sistema semidinâmico $\pi: X \times \mathbb{R}_{+} \rightarrow X$ dado por

$$
\pi((r, y), t)=(r+t, y)
$$

Sejam $M=\{0\} \times Y$ e $I: M \rightarrow X$ dado por

$$
I(0, y)=(-1, \sigma(y))
$$

Observemos que $I$ é contínuo. Dado $z=(-1, y) \in X$, consideremos as seguintes notações

$$
z_{n+1}=\left(0, \sigma^{n}(y)\right) \quad \text { e } \quad I\left(z_{n+1}\right)=z_{n+1}^{+}=\left(-1, \sigma^{n+1}(y)\right),
$$

para $n=0,1,2, \ldots$, onde $z_{0}^{+}=z$.

Dados $t, s \in \mathbb{R}_{+}$, existem $k, m \in \mathbb{N}$ tais que $t=k+t^{\prime} \operatorname{com} 0 \leq t^{\prime}<1$ e $s=m+s^{\prime} \operatorname{com} 0 \leq s^{\prime}<1$. Então

$$
\begin{gathered}
d(\tilde{\pi}(z, t), \tilde{\pi}(z, s))=d\left(\pi\left(z_{k}^{+}, t^{\prime}\right), \pi\left(z_{m}^{+}, s^{\prime}\right)\right)=d\left(\pi\left(\left(-1, \sigma^{k}(y)\right), t^{\prime}\right), \pi\left(\left(-1, \sigma^{m}(y)\right), s^{\prime}\right)\right)= \\
=d\left(\left(t^{\prime}-1, \sigma^{k}(y)\right),\left(s^{\prime}-1, \sigma^{m}(y)\right)\right)=\max \left\{\left|t^{\prime}-s^{\prime}\right|, d_{Y}\left(\sigma^{k}(y), \sigma^{m}(y)\right)\right\} .
\end{gathered}
$$


Afirmação 1: $z \in \widetilde{L}^{+}(z)$.

De fato, seja $\varepsilon>0$ arbitrário. Como y é um ponto recorrente de $\sigma$, existe uma sequência $\left\{n_{k}\right\}_{k \geq 1} \subset \mathbb{N}$ tal que $n_{k} \stackrel{k \rightarrow+\infty}{\longrightarrow}+\infty$ e $d_{Y}\left(\sigma^{n_{k}}(y), y\right)<\varepsilon$ para todo $k \in \mathbb{N}^{*}$. Então

$$
d\left(\tilde{\pi}\left(z, n_{k}\right), z\right)=\max \left\{0, d_{Y}\left(\sigma^{n_{k}}(y), y\right)\right\}<\varepsilon,
$$

para todo $k \in \mathbb{N}^{*}$. Portanto, $z \in \widetilde{L}^{+}(z)$.

Afirmação 2: $z$ não é fracamente quase $\widetilde{\pi}$-periódico.

Como y não é quase periódico, existe $\varepsilon_{0}>0$ tal que para todo $N \in \mathbb{N}$ podemos encontrar um número $n_{N} \in \mathbb{Z}_{+}$satisfazendo a desigualdade

$$
d_{Y}\left(\sigma^{n_{N}+i}(y), y\right) \geq \varepsilon_{0},
$$

para todo $i \in\{0,1,2, \ldots, N\}$.

Agora, dado $T>0$, existe um número natural $N_{T}>0$ tal que $N_{T} \leq T<N_{T}+1$. Seja $n_{N_{T}}:=n_{T}$ dado por (4.2). Para qualquer $s \in\left[n_{T}, n_{T}+T\right]$, podemos escrever $s=n_{T}+i_{0}+s^{\prime}$ para algum $i_{0} \in\left\{0,1,2, \ldots, N_{T}\right\}$ e $0 \leq s^{\prime}<1$. Então

$$
d(z, \tilde{\pi}(z, s))=\max \left\{s^{\prime}, d_{Y}\left(y, \sigma^{n_{T}+i_{0}}(y)\right)\right\} \geq \varepsilon_{0} .
$$

Portanto, $z$ não é fracamente quase $\widetilde{\pi}$-periódico.

No caso contínuo, se um ponto é positivamente Lyapunov estável e recorrente então este ponto é quase periódico, veja [27, Teorema 8.11]. A seguir, apresentamos o conceito de estabilidade de Lyapunov para sistemas semidinâmicos impulsivos e obtemos condições suficientes para que um ponto $\tilde{\pi}$-recorrente seja também fracamente quase $\tilde{\pi}$-periódico.

O conceito de estabilidade de Lyapunov para sistemas impulsivos foi definido por Kaul em [22]. Veja a definição seguinte.

Definição 4.1.6 Um ponto $x \in X$ é denominado Lyapunov $\widetilde{\pi}$-estável, em relação a um subcon- 
junto $B \subset X$, se, dado qualquer $\varepsilon>0$, existe $\delta>0$ tal que para qualquer $y \in B$ satisfazendo a condição $d(x, y)<\delta$ implica em

$$
d(\tilde{\pi}(x, t), \tilde{\pi}(y, t))<\varepsilon \quad \text { para todo } \quad t \in L(x, \varepsilon) .
$$

Temos o seguinte resultado.

Teorema 4.1.7 [14, Teorema 4.3] Sejam $(X, \pi ; M, I)$ um sistema semidinâmico impulsivo e $x \in X \backslash M$. Suponha que $\overline{\tilde{\pi}^{+}(x)}$ seja compacto. Se $x$ é $\tilde{\pi}$-recorrente e Lyapunov $\tilde{\pi}$-estável em relação ao conjunto $\tilde{\pi}^{+}(x)$, então x é fracamente quase $\tilde{\pi}$-periódico.

Demonstração: Seja $\varepsilon>0$. Como $x$ é Lyapunov $\tilde{\pi}$-estável em relação ao conjunto $\widetilde{\pi}^{+}(x)$, existe $\delta>0$ tal que se $y \in \tilde{\pi}^{+}(x)$ e $d(x, y)<\delta$, então

$$
d(\tilde{\pi}(x, t), \tilde{\pi}(y, t))<\varepsilon \quad \text { para todo } \quad t \in L(x, \varepsilon) .
$$

Como $x$ é $\tilde{\pi}$-recorrente, usando o Teorema 3.1.6, podemos concluir que o conjunto

$$
K_{\delta}=\{\tau>0: d(x, \tilde{\pi}(x, \tau))<\delta\}
$$

é relativamente denso, ou seja, existe $T>0$ tal que para qualquer $\alpha \geq 0$, o intervalo $[\alpha, \alpha+T]$ contém um número $\tau>0$ satisfazendo $d(x, \tilde{\pi}(x, \tau))<\delta$. Então por (4.3) obtemos

$$
d(\tilde{\pi}(x, t), \tilde{\pi}(x, t+\tau))<\varepsilon \quad \text { para todo } \quad t \in L(x, \varepsilon) .
$$

Isto mostra que $x$ é fracamente quase $\widetilde{\pi}$-periódico.

Definição 4.1.8 Dizemos que a órbita $\tilde{\pi}^{+}(x), x \in X$, aproxima-se uniformemente de um conjunto $\mathscr{A}$, se para qualquer $\varepsilon>0$, existe $T=T(\varepsilon)>0$ tal que

$$
\mathscr{A} \subset B(\tilde{\pi}(x,[t, t+T]), \varepsilon), \quad \text { para todo } \quad t \in \mathbb{R}_{+} .
$$

Agora, vamos apresentar uma generalização de um resultado apresentado em [5]. Em [5, Teorema 3.3], é provado que se $x \in X \backslash M$ é um ponto tal que $\overline{\widetilde{\pi}^{+}(x)}$ é compacto e $\widetilde{L}^{+}(x) \cap$ $M=\emptyset$, então $\widetilde{L}^{+}(x)$ é minimal se, e somente se, $\widetilde{\pi}^{+}(x)$ aproxima-se uniformemente do conjunto $\widetilde{L}^{+}(x)$. Mostraremos no Teorema 4.1.11 que este resultado continua válido se eliminarmos as condições $x \notin M$ e $\widetilde{L}^{+}(x) \cap M=\emptyset$. 
Consideremos os seguinte resultado auxiliar.

Lema 4.1.9 [14, Lema 4.1] Sejam $(X, \pi ; M, I)$ um sistema semidinâmico impulsivo e A um subconjunto fechado de $X$ tal que $A \backslash M$ é positivamente $\tilde{\pi}$-invariante. Seja a $\in A \backslash M$ e $\left\{w_{n}\right\}_{n \geq 1}$ uma sequência em $X$ tal que $w_{n} \stackrel{n \rightarrow+\infty}{\longrightarrow}$ a. Se $\left\{\tau_{n}\right\}_{n \geq 1}$ é uma sequência limitada em $\mathbb{R}_{+}$, então dado $\varepsilon>0$ existem uma subsequência $\left\{\tau_{n_{\ell}}\right\}_{\ell \geq 1}$ de $\left\{\tau_{n}\right\}_{n \geq 1}$, um número $n_{0} \in \mathbb{N}$ e $\bar{a} \in A$ tais que $d\left(\tilde{\pi}\left(w_{n_{\ell}}, \tau_{n_{\ell}}\right), \bar{a}\right)<\varepsilon$ para todo $n_{\ell} \geq n_{0}$.

Demonstração: Seja $\varepsilon>0$. Inicialmente, notemos que existe uma subsequência convergente $\left\{\tau_{n_{\ell}}\right\}_{\ell \geq 1}$ de $\left\{\tau_{n}\right\}_{n \geq 1}$, tal que $\tau_{n_{\ell}} \stackrel{\ell \rightarrow+\infty}{\longrightarrow} \tau \in \mathbb{R}_{+}$. Temos três casos a considerar.

Case 1) $0 \leq \tau<\phi(a)$

Como $a \notin M$, temos $\phi\left(w_{n}\right) \stackrel{n \rightarrow+\infty}{\longrightarrow} \phi(a)$. Segue que, existe um número $p_{0} \in \mathbb{N}$ tal que

$$
0 \leq \tau_{n_{\ell}}<\phi\left(w_{n_{\ell}}\right)
$$

para todo $n_{\ell} \geq p_{0}$. Assim,

$$
\tilde{\pi}\left(w_{n_{\ell}}, \tau_{n_{\ell}}\right)=\pi\left(w_{n_{\ell}}, \tau_{n_{\ell}}\right) \stackrel{\ell \rightarrow+\infty}{\longrightarrow} \pi(a, \tau)=\tilde{\pi}(a, \tau) .
$$

Considere $\bar{a}=\tilde{\pi}(a, \tau)$. Como $A \backslash M$ é positivamente $\tilde{\pi}$-invariante, temos que $\bar{a} \in A$. Então, existe $n_{0} \in \mathbb{N}$ tal que $d\left(\tilde{\pi}\left(w_{n_{\ell}}, \tau_{n_{\ell}}\right), \bar{a}\right)<\varepsilon$ para todo $n_{\ell} \geq n_{0}$.

Case 2) $\tau>\phi(a)$ e $\tau \neq \sum_{j=0}^{r} \phi\left(a_{j}^{+}\right)$para todo $r=1,2, \ldots$

Neste caso, existe um número $k \in \mathbb{N}$ tal que

$$
\tau=\sum_{j=0}^{k} \phi\left(a_{j}^{+}\right)+s \quad \text { com } \quad 0<s<\phi\left(a_{k+1}^{+}\right) .
$$

Note que $\tilde{\pi}(a, \tau)=\pi\left(a_{k+1}^{+}, s\right)$. Pela continuidade de $\pi$ e $I$ temos $\left(w_{n}\right)_{j}^{+} \stackrel{n \rightarrow+\infty}{\longrightarrow} a_{j}^{+}$para todo $j \in \mathbb{N}$. Como $\phi$ é contínua em $X \backslash M$, existe uma sequência $\left\{s_{n_{\ell}}\right\}_{\ell \geq 1} \subset \mathbb{R}_{+}$tal que

$$
\tau_{n_{\ell}}=\sum_{j=0}^{k} \phi\left(\left(w_{n_{\ell}}\right)_{j}^{+}\right)+s_{n_{\ell}} \quad \text { com } \quad s_{n_{\ell}} \stackrel{\ell \rightarrow+\infty}{\longrightarrow} s .
$$


Além disso, $\tilde{\pi}\left(w_{n_{\ell}}, \tau_{n_{\ell}}\right)=\pi\left(\left(w_{n_{\ell}}\right)_{k+1}^{+}, s_{n_{\ell}}\right)$ para todo $\ell \in \mathbb{N}^{*}$. Então

$$
\tilde{\pi}\left(w_{n_{\ell}}, \tau_{n_{\ell}}\right)=\pi\left(\left(w_{n_{\ell}}\right)_{k+1}^{+}, s_{n_{\ell}}\right) \stackrel{\ell \rightarrow+\infty}{\longrightarrow} \pi\left(a_{k+1}^{+}, s\right)=\tilde{\pi}(a, \tau) .
$$

Se tomarmos $\bar{a}=\tilde{\pi}(a, \tau) \in A$, o resultado segue.

Case 3) $\tau=\sum_{j=0}^{k} \phi\left(a_{j}^{+}\right)$para algum $k=0,1,2, \ldots$

Neste último caso, é suficiente considerarmos duas possibilidades, ou seja, quando $\left\{\tau_{n_{\ell}}\right\}_{\ell \geq 1}$ admite uma subsequência $\left\{\tau_{n_{\ell}}^{\prime}\right\}_{\ell \geq 1}$ tal que $\tau_{n_{\ell}}^{\prime}<\sum_{j=0}^{k} \phi\left(a_{j}^{+}\right)$para todo $\ell \in \mathbb{N}^{*}$ e quando $\left\{\tau_{n_{\ell}}\right\}_{n \geq 1}$ admite uma subsequência $\left\{\tau_{n_{\ell}}^{\prime \prime}\right\}_{\ell \geq 1}$ tal que $\tau_{n_{\ell}}^{\prime \prime} \geq \sum_{j=0}^{k} \phi\left(a_{j}^{+}\right)$para todo $\ell \in \mathbb{N}^{*}$.

Suponhamos, primeiramente, que exista uma subsequência $\left\{\tau_{n_{\ell}}^{\prime}\right\}_{\ell \geq 1}$ tal que $\tau_{n_{\ell}}^{\prime}<\sum_{j=0}^{k} \phi\left(a_{j}^{+}\right)$ para todo $\ell \in \mathbb{N}^{*}$. Então segue que

$$
\tilde{\pi}\left(w_{n_{\ell}}, \tau_{n_{\ell}}^{\prime}\right) \stackrel{\ell \rightarrow+\infty}{\longrightarrow} a_{k+1} \in A\left(A \text { é fechado e } \tilde{\pi}^{+}(a) \subset A\right)
$$

Assim, existe $n_{0}^{\prime} \in \mathbb{N}$ tal que

$$
d\left(\widetilde{\pi}\left(w_{n_{\ell}}, \tau_{n_{\ell}}^{\prime}\right), a_{k+1}\right)<\varepsilon \quad \text { para todo } \quad n_{\ell} \geq n_{0}^{\prime} .
$$

Agora, se existe uma subsequência $\left\{\tau_{n_{\ell}}^{\prime \prime}\right\}_{\ell \geq 1}$ tal que $\tau_{n_{\ell}}^{\prime \prime} \geq \sum_{j=0}^{k} \phi\left(a_{j}^{+}\right)$para todo $\ell \in \mathbb{N}^{*}$, então $\tilde{\pi}\left(w_{n_{\ell}}, \tau_{n_{\ell}}^{\prime \prime}\right) \stackrel{\ell \rightarrow+\infty}{\longrightarrow} a_{k+1}^{+}=I\left(a_{k+1}\right) \in A\left(\tilde{\pi}^{+}(a) \subset A\right.$ pois $A \backslash M$ é positivamente $\tilde{\pi}$-invariante $)$. Logo, existe $n_{0}^{\prime \prime} \in \mathbb{N}$ tal que

$$
d\left(\tilde{\pi}\left(w_{n_{\ell}}, \tau_{n_{\ell}}^{\prime \prime}\right), a_{k+1}^{+}\right)<\varepsilon \quad \text { para todo } \quad n_{\ell} \geq n_{0}^{\prime \prime} .
$$

Portanto, em todos os casos acima, podemos encontrar um elemento $\bar{a} \in A$, uma sequência $\left\{\tau_{n_{\ell}}\right\}_{\ell \geq 1}$ e um número natural $n_{0}$ tal que $d\left(\tilde{\pi}\left(w_{n_{\ell}}, \tau_{n_{\ell}}\right), \bar{a}\right)<\varepsilon$ para todo $n_{\ell} \geq n_{0}$.

O teorema a seguir apresenta condições suficientes para que o conjunto limite positivo de um ponto seja minimal. 
Teorema 4.1.10 [14, Teorema 4.4] Sejam $(X, \pi ; M, I)$ um sistema semidinâmico impulsivo e $x \in X$ tal que $\overline{\widetilde{\pi}^{+}(x)}$ é compacto. Se $\widetilde{\pi}^{+}(x)$ aproxima-se uniformemente de $\widetilde{L}^{+}(x)$, então $\widetilde{L}^{+}(x)$ é um conjunto minimal. Além disso, se um ponto $y \in \widetilde{L}^{+}(x) \backslash M$ é Lyapunov $\tilde{\pi}$-estável em relação a $\tilde{\pi}^{+}(y)$, então y é fracamente quase $\tilde{\pi}$-periódico.

Demonstração: Mostremos, inicialmente, que $\widetilde{L}^{+}(x)$ é minimal. Suponhamos o contrário, isto é, que exista um subconjunto fechado $A \varsubsetneqq \widetilde{L}^{+}(x)$ tal que $A \backslash M \neq \emptyset$ e $A \backslash M$ é positivamente $\tilde{\pi}$ invariante. Seja $w \in \widetilde{L}^{+}(x)$ tal que $w \notin A$ e considere $\varepsilon=\frac{d(w, A)}{3}>0$.

Como $\widetilde{\pi}^{+}(x)$ aproxima-se uniformemente de $\widetilde{L}^{+}(x)$, existe $T=T(\varepsilon)>0$ tal que

$$
\widetilde{L}^{+}(x) \subset B(\tilde{\pi}(x,[t, t+T]), \varepsilon) \quad \text { para todo } t \in \mathbb{R}_{+} .
$$

Seja $a \in A \backslash M \subset \widetilde{L}^{+}(x)$, logo existe uma sequência $\left\{t_{n}\right\}_{n \geq 1} \subset \mathbb{R}_{+}$tal que $t_{n} \stackrel{n \rightarrow+\infty}{\longrightarrow}+\infty$ e $\tilde{\pi}\left(x, t_{n}\right) \stackrel{n \rightarrow+\infty}{\longrightarrow} a$. De (4.4) temos, em particular, que

$$
\widetilde{L}^{+}(x) \subset B\left(\tilde{\pi}\left(x,\left[t_{n}, t_{n}+T\right]\right), \varepsilon\right), \quad \text { para todo } n \in \mathbb{N}^{*}
$$

Logo

$$
w \in B\left(\tilde{\pi}\left(x,\left[t_{n}, t_{n}+T\right]\right), \varepsilon\right), \quad \text { para todo } n \in \mathbb{N}^{*}
$$

Assim, para cada $n \in \mathbb{N}^{*}$, existe $\tau_{n} \in[0, T]$ tal que

$$
d\left(w, \tilde{\pi}\left(x, t_{n}+\tau_{n}\right)\right)<\varepsilon
$$

Como $[0, \mathrm{~T}]$ é compacto, podemos supor que $\tau_{n} \stackrel{n \rightarrow+\infty}{\longrightarrow} \tau \in[0, T]$. Defina $w_{n}=\tilde{\pi}\left(x, t_{n}\right), n \in \mathbb{N}^{*}$. Pelo Lema 4.1.9, existem $\bar{a} \in A$, uma subsequência $\left\{\tau_{n_{\ell}}\right\}_{\ell \geq 1}$ e um número $n_{0} \in \mathbb{N}$ tal que

$$
d\left(\tilde{\pi}\left(w_{n_{\ell}}, \tau_{n_{\ell}}\right), \bar{a}\right)<\varepsilon
$$

para todo $n_{\ell} \geq n_{0}$. Então

$$
3 \varepsilon=d(w, A) \leq d(w, \bar{a}) \leq d\left(w, \widetilde{\pi}\left(w_{n_{0}}, \tau_{n_{0}}\right)\right)+d\left(\tilde{\pi}\left(w_{n_{0}}, \tau_{n_{0}}\right), \bar{a}\right)<\varepsilon+\varepsilon=2 \varepsilon,
$$

o que é uma contradição. Portanto concluímos que $\widetilde{L}^{+}(x)$ é minimal. 
Por outro lado, notemos que $\widetilde{L}^{+}(x)$ é compacto, pois $\widetilde{L}^{+}(x) \subset \overline{\widetilde{\pi}^{+}(x)}$. Então pelo Teorema 3.1.3 todo ponto $y \in \widetilde{L}^{+}(x) \backslash M$ é $\tilde{\pi}$-recorrente. Pela hipótese $y \in \widetilde{L}^{+}(x) \backslash M$ é Lyapunov $\widetilde{\pi}$-estável em relação a $\widetilde{\pi}^{+}(y)$, então usando o Teorema 4.1 .7 segue que y é fracamente quase $\tilde{\pi}$-periódico.

Teorema 4.1.11 [14, Teorema 4.5] Sejam $(X, \pi ; M, I)$ um sistema semidinâmico impulsivo e $x \in X$ tal que $\overline{\tilde{\pi}^{+}(x)}$ é compacto. Então $\widetilde{L}^{+}(x)$ é um conjunto minimal se, e somente se, $\tilde{\pi}^{+}(x)$ aproxima-se uniformemente de $\widetilde{L}^{+}(x)$.

Demonstração: A condição necessária segue do Teorema 4.1.10. Vamos mostrar a condição suficiente. Suponhamos que $\widetilde{\pi}^{+}(x)$ não aproxima-se uniformemente de $\widetilde{L}^{+}(x)$, isto é, existem $\varepsilon>0$ e sequências $\left\{T_{n}\right\}_{n \geq 1},\left\{t_{n}\right\}_{n \geq 1}, \subset \mathbb{R}_{+},\left\{y_{n}\right\}_{n \geq 1} \subset \widetilde{L}^{+}(x)$ tais que $T_{n} \stackrel{n \rightarrow+\infty}{\longrightarrow}+\infty \mathrm{e}$

$$
d\left(y_{n}, \tilde{\pi}\left(x,\left[t_{n}, t_{n}+T_{n}\right]\right)\right) \geq \varepsilon \text { para todo } n \in \mathbb{N}^{*}
$$

Observemos que $\widetilde{L}^{+}(x)$ é compacto, pois $\widetilde{L}^{+}(x) \subset \overline{\widetilde{\pi}^{+}(x)}$, logo podemos supor que existe $y \in \widetilde{L}^{+}(x)$ tal que

$$
y_{n} \stackrel{n \rightarrow+\infty}{\longrightarrow} y .
$$

Além disso, como para cada $n \in \mathbb{N}^{*}, y_{n} \in \widetilde{L}^{+}(x)$, então existe uma sequência $\left\{\tau_{m}^{n}\right\}_{m \geq 1} \subset \mathbb{R}_{+}$tal que

$$
\tau_{m}^{n} \stackrel{m \rightarrow+\infty}{\longrightarrow}+\infty \quad \text { e } \quad \tilde{\pi}\left(x, \tau_{m}^{n}\right) \stackrel{m \rightarrow+\infty}{\longrightarrow} y_{n}
$$

Assim, de (4.7) segue que existe $m_{n}>n$ tal que

$$
d\left(\tilde{\pi}\left(x, \tau_{m_{n}}^{n}\right), y_{n}\right)<\frac{\varepsilon}{2}
$$

para todo $n \in \mathbb{N}^{*}$. Além disso, de (4.6) e (4.7) obtemos

$$
\tilde{\pi}\left(x, \tau_{m_{n}}^{n}\right) \stackrel{n \rightarrow+\infty}{\longrightarrow} y
$$

Por outro lado, $\left\{\tilde{\pi}\left(x, t_{n}+\frac{T_{n}}{2}\right)\right\}_{n \geq 1} \subset \overline{\tilde{\pi}^{+}(x)},\left(t_{n}+\frac{T_{n}}{2}\right) \stackrel{n \rightarrow+\infty}{\longrightarrow}+\infty$ e como $\overline{\tilde{\pi}^{+}(x)}$ é compacto podemos assumir que existe $b \in \widetilde{L}^{+}(x)$ tal que

$$
\tilde{\pi}\left(x, t_{n}+\frac{T_{n}}{2}\right) \stackrel{n \rightarrow+\infty}{\longrightarrow} b
$$


Na sequência, vamos mostrar que existe um elemento $z \in \widetilde{L}^{+}(x) \backslash M$ tal que $y \notin \overline{\widetilde{\pi}^{+}(z)}$. Isto vai contradizer a minimalidade de $\widetilde{L}^{+}(x)$ e, portanto, o teorema estará provado. Temos dois casos a considerar:

Caso 1) $b \notin M$. Seja $t \geq 0$ arbitrário e fixado tal que $t \neq \sum_{j=0}^{k} \phi\left(b_{j}^{+}\right)$para todo $k=0,1,2, \ldots$ Neste caso, de (4.10) e pelo Lema 1.4.6 temos

$$
\tilde{\pi}\left(x, t_{n}+\frac{T_{n}}{2}+t\right) \stackrel{n \rightarrow+\infty}{\longrightarrow} \tilde{\pi}(b, t) .
$$

Como $T_{n} \stackrel{n \rightarrow+\infty}{\longrightarrow}+\infty$, existe $n_{0} \in \mathbb{N}$ tal que $\frac{T_{n}}{2}>t$ para $n \geq n_{0}$. Então $t_{n}<t_{n}+\frac{T_{n}}{2}+t<t_{n}+T_{n}$ para todo $n \geq n_{0}$. Logo, usando (4.5) e (4.8) obtemos

$$
\begin{aligned}
d\left(\tilde{\pi}\left(x, t_{n}+\frac{T_{n}}{2}+t\right), \tilde{\pi}\left(x, \tau_{m_{n}}^{n}\right)\right) & \geq d\left(y_{n}, \tilde{\pi}\left(x, t_{n}+\frac{T_{n}}{2}+t\right)\right)-d\left(y_{n}, \tilde{\pi}\left(x, \tau_{m_{n}}^{n}\right)\right) \\
& \geq \varepsilon-\frac{\varepsilon}{2}=\frac{\varepsilon}{2}
\end{aligned}
$$

para todo $n \geq n_{0}$. Fazendo $n \rightarrow+\infty$ na desigualdade acima e usando (4.9) e (4.11), obtemos $d(\tilde{\pi}(b, t), y) \geq \frac{\varepsilon}{2}$. Como $t$ é arbitrário, concluímos que

$$
d(\widetilde{\pi}(b, t), y) \geq \frac{\varepsilon}{2} \quad \text { para todo } \quad t \neq \sum_{j=0}^{k} \phi\left(b_{j}^{+}\right), k=0,1,2, \ldots
$$

Se $t=\sum_{j=0}^{k} \phi\left(b_{j}^{+}\right)$para algum $k=0,1,2, \ldots$. Podemos tomar uma sequência de números reais positivos $\left\{\lambda_{n}\right\}_{n \geq 1}$ tal que

$$
\lambda_{n} \stackrel{n \rightarrow+\infty}{\longrightarrow} \sum_{j=0}^{k} \phi\left(b_{j}^{+}\right), \quad \text { com } \quad \sum_{j=0}^{k} \phi\left(b_{j}^{+}\right)<\lambda_{n}<\sum_{j=0}^{k+1} \phi\left(b_{j}^{+}\right), n \in \mathbb{N}^{*}
$$

Daí por (4.12) temos

$$
d\left(\tilde{\pi}\left(b, \lambda_{n}\right), y\right) \geq \frac{\varepsilon}{2} \text { para todo } n \in \mathbb{N}^{*} .
$$

Fazendo $n \rightarrow+\infty$ e usando a continuidade à direita de $\tilde{\pi}$, obtemos

$$
d\left(\tilde{\pi}\left(b, \sum_{j=0}^{k} \phi\left(b_{j}^{+}\right)\right), y\right) \geq \frac{\varepsilon}{2}
$$


Então, concluímos que

$$
d(\tilde{\pi}(b, t), y) \geq \frac{\varepsilon}{2} \text { para todo } t \geq 0,
$$

e portanto, $y \notin \overline{\widetilde{\pi}^{+}(b)}$. Isto é uma contradição, pois $b \in \widetilde{L}^{+}(x) \backslash M$ e $\widetilde{L}^{+}(x)$ é minimal implicando em $\overline{\widetilde{\pi}^{+}(b)}=\widetilde{L}^{+}(x)$, veja Teorema 2.1.3.

Caso 2) $b \in M$. Como $M$ satisfaz a condição STC, existe um STC-tubo $F(L,[0,2 \lambda])$ através de $b$ dado por uma seção $S$. Além disso, o tubo é uma vizinhança de $b, \operatorname{logo}$ existe um $\eta>0$ tal que

$$
B(b, \eta) \subset F(L,[0,2 \lambda])
$$

Chamemos de $H_{1}=F(L,(\lambda, 2 \lambda]) \cap B(b, \eta)$ e $H_{2}=F(L,[0, \lambda]) \cap B(b, \eta)$. Seja $w_{n}=\tilde{\pi}\left(x, t_{n}+\frac{T_{n}}{2}\right)$, $n \in \mathbb{N}^{*}$. Então por (4.10) temos $w_{n} \stackrel{n \rightarrow+\infty}{\longrightarrow} b$.

Podemos assumir, sem perda de generalidade (tomando uma subsequência, se for preciso), que $\left\{w_{n}\right\}_{n \geq 1} \subset H_{1}$ ou $\left\{w_{n}\right\}_{n \geq 1} \subset H_{2}$.

- Suponhamos primeiro que $\left\{w_{n}\right\}_{n \geq 1} \subset H_{1}$. Observe que $\phi\left(w_{n}\right) \stackrel{n \rightarrow+\infty}{\longrightarrow} 0, \operatorname{logo}$

$$
\tilde{\pi}\left(w_{n}, \phi\left(w_{n}\right)\right) \stackrel{n \rightarrow+\infty}{\longrightarrow} I(b),
$$

isto é,

$$
\tilde{\pi}\left(x, t_{n}+\frac{T_{n}}{2}+\phi\left(w_{n}\right)\right) \stackrel{n \rightarrow+\infty}{\longrightarrow} I(b) .
$$

Mostraremos que $y \notin \overline{\widetilde{\pi}^{+}(I(b))}$. Note que $I(b) \in \widetilde{L}^{+}(x) \backslash M$, pois $\left(t_{n}+\frac{T_{n}}{2}+\phi\left(w_{n}\right)\right) \stackrel{n \rightarrow+\infty}{\longrightarrow}+\infty$ e $I(M) \cap M=\emptyset$. Seja $t \geq 0$ tal que $t \neq \sum_{j=0}^{k} \phi\left(I(b)_{j}^{+}\right)$para todo $k=0,1,2, \ldots$ De (4.13) e pelo Lema 1.4.6 temos

$$
\tilde{\pi}\left(x, t_{n}+\frac{T_{n}}{2}+\phi\left(w_{n}\right)+t\right) \stackrel{n \rightarrow+\infty}{\longrightarrow} \tilde{\pi}(I(b), t) .
$$

Por outro lado, existe $n_{0}^{\prime} \in \mathbb{N}$ tal que $\frac{T_{n}}{2}>t+\phi\left(w_{n}\right)$ para todo $n \geq n_{0}^{\prime}$. Então, $t_{n}<t_{n}+\frac{T_{n}}{2}+\phi\left(w_{n}\right)+$ $t<t_{n}+T_{n}$ para todo $n \geq n_{0}^{\prime}$. Logo, usando (4.5) e (4.8) obtemos a seguinte desigualdade

$$
\begin{aligned}
d\left(\tilde{\pi}\left(x, t_{n}+\frac{T_{n}}{2}+\phi\left(w_{n}\right)+t\right), \tilde{\pi}\left(x, \tau_{m_{n}}^{n}\right)\right) & \geq d\left(y_{n}, \tilde{\pi}\left(x, t_{n}+\frac{T_{n}}{2}+\phi\left(w_{n}\right)+t\right)\right)-d\left(y_{n}, \tilde{\pi}\left(x, \tau_{m_{n}}^{n}\right)\right) \\
& \geq \varepsilon-\frac{\varepsilon}{2}=\frac{\varepsilon}{2},
\end{aligned}
$$


para todo $n \geq n_{0}^{\prime}$. Fazendo $n \rightarrow+\infty$ na desigualdade acima e usando (4.9) e (4.14) segue que $d(\widetilde{\pi}(I(b), t), y) \geq \frac{\varepsilon}{2}$. Como $t$ é arbitrário, concluímos que

$$
d(\tilde{\pi}(I(b), t), y) \geq \frac{\varepsilon}{2} \quad \text { para todo } \quad t \neq \sum_{j=0}^{k} \phi\left(I(b)_{j}^{+}\right), k=0,1,2, \ldots
$$

Agora, se $t=\sum_{j=0}^{k} \phi\left(I(b)_{j}^{+}\right)$para algum $k=0,1,2, \ldots$, consideramos uma sequência de números reais positivos $\left\{\lambda_{n}\right\}_{n \geq 1}$, tal que

$$
\lambda_{n} \stackrel{n \rightarrow+\infty}{\longrightarrow} \sum_{j=0}^{k} \phi\left(I(b)_{j}^{+}\right), \quad \text { com } \sum_{j=0}^{k} \phi\left(I(b)_{j}^{+}\right)<\lambda_{n}<\sum_{j=0}^{k+1} \phi\left(I(b)_{j}^{+}\right) .
$$

Daí, usando (4.15), temos

$$
d\left(\tilde{\pi}\left(I(b), \lambda_{n}\right), y\right) \geq \frac{\varepsilon}{2} \text { para todo } n \in \mathbb{N}^{*}
$$

Fazendo $n \rightarrow+\infty$, segue da continuidade à direita de $\tilde{\pi}$ que

$$
d\left(\tilde{\pi}\left(I(b), \sum_{j=0}^{k} \phi\left(I(b)_{j}^{+}\right)\right), y\right) \geq \frac{\varepsilon}{2}
$$

Assim $d(\widetilde{\pi}(I(b), t), y) \geq \frac{\varepsilon}{2}$ para todo $t \geq 0$, de onde obtemos

$$
y \notin \overline{\widetilde{\pi}^{+}(I(b))} .
$$

Novamente, isso é uma contradição, pois $I(b) \in \widetilde{L}^{+}(x) \backslash M$ e assim $\overline{\widetilde{\pi}^{+}(I(b))}=\widetilde{L}^{+}(x)$ já que $\widetilde{L}^{+}(x)$ é minimal.

- Suponhamos, agora, que $\left\{w_{n}\right\}_{n \geq 1} \subset H_{2}$. Tome $0<\beta<\phi(b)$. Como $w_{n} \stackrel{n \rightarrow+\infty}{\longrightarrow} b$, temos

$$
\tilde{\pi}\left(w_{n}, \beta\right) \stackrel{n \rightarrow+\infty}{\longrightarrow} \tilde{\pi}(b, \beta),
$$

ou seja,

$$
\tilde{\pi}\left(x, t_{n}+\frac{T_{n}}{2}+\beta\right) \stackrel{n \rightarrow+\infty}{\longrightarrow} \tilde{\pi}(b, \beta)
$$


Observe que $b_{1}=\tilde{\pi}(b, \beta) \in \widetilde{L}^{+}(x) \backslash M$, pois $\left(t_{n}+\frac{T_{n}}{2}+\beta\right) \stackrel{n \rightarrow+\infty}{\longrightarrow}+\infty$ e $0<\beta<\phi(b)$.

Logo, se considerarmos o semifluxo $\tilde{\pi}\left(b_{1}, t\right)$ e seguindo os mesmos passos anteriores, podemos obter a seguinte desigualdade $d\left(\tilde{\pi}\left(b_{1}, t\right), y\right) \geq \frac{\varepsilon}{2}$ para todo $t \geq 0$, ou seja,

$$
y \notin \overline{\widetilde{\pi}^{+}\left(b_{1}\right)}
$$

o que é uma contradição.

Portanto, o teorema está provado.

\subsection{Quase estabilidade de Zhukovskij}

Nesta seção, vamos estudar a teoria de quase estabilidade de Zhukovskij. Em sistemas semidinâmicos impulsivos, este tipo de estabilidade foi inicialmente introduzido por Changming Ding em [19]. Começamos esta seção apresentando a definição de reparametrização, na sequência definimos o conceito de quase estabilidade de Zhukovskij e apresentamos também alguns resultados envolvendo minimalidade, recorrência e periodicidade. No último resultado desta seção, apresentamos condições suficientes para que o conjunto limite positivo de um ponto seja um atrator. O leitor pode encontrar outros resultados sobre quase estabilidade de Zhukovskij em [19].

Definição 4.2.1 Uma reparametrização é um homeomorfismo $h: \mathbb{R}_{+} \rightarrow \mathbb{R}_{+}$tal que $h(0)=0$.

Definição 4.2.2 Um ponto $x \in X \backslash M$ é chamado Zhukovskij quase $\widetilde{\pi}$-estável se dado $\varepsilon>0$, existe um $\delta=\delta(x, \varepsilon)>0$, tal que se $y \in X$ e $d(y, x)<\delta$ então podemos encontrar uma reparametrização $\tau_{y}$ tal que

$$
d\left(\tilde{\pi}(x, t), \tilde{\pi}\left(y, \tau_{y}(t)\right)\right)<\varepsilon, \text { para todo } t \geq 0
$$

Além disso, se existe $\lambda>0$ tal que $d(y, x)<\lambda$ implica $d\left(\tilde{\pi}(x, t), \tilde{\pi}\left(y, \tau_{y}(t)\right) \stackrel{t \rightarrow+\infty}{\longrightarrow} 0\right.$, então $x$ é chamado assintoticamente Zhukovskij quase $\tilde{\pi}$-estável.

Um subconjunto $A \subset X \backslash M$ é chamado Zhukovskij (assintoticamente Zhukovskij) quase $\tilde{\pi}$-stável se cada ponto $y \in A$ possui esta propriedade. 
Definição 4.2.3 Um ponto $x \in X \backslash M$ é uniformemente assintoticamente Zhukovskij quase $\tilde{\pi}$ estável se dado $\varepsilon>0$, existe $\delta=\delta(\varepsilon)>0$ tal que para cada $s \geq 0$ e $d(y, \tilde{\pi}(x, s))<\delta$, então podemos encontrar uma reparametrização $\tau_{y}$ tal que $d\left(\tilde{\pi}(x, s+t), \tilde{\pi}\left(y, \tau_{y}(t)\right)\right)<\varepsilon$ para todo $t \geq 0$ e, além disso, $d\left(\tilde{\pi}(x, s+t), \tilde{\pi}\left(y, \tau_{y}(t)\right)\right) \stackrel{t \rightarrow+\infty}{\longrightarrow} 0$.

Um subconjunto $A \subset X \backslash M$ é chamado uniformemente assintoticamente Zhukovskij quase $\tilde{\pi}$-stável se cada ponto $y \in A$ possui esta propriedade.

Observação 4.2.4 Como foi observado em [19], se tomarmos $s=\tau_{y}(t)$ e $h(s)=\tau_{y}^{-1}(s)=t$, então podemos escrever

$$
d\left(\tilde{\pi}(x, t), \tilde{\pi}\left(y, \tau_{y}(t)\right)\right)=d(\tilde{\pi}(x, h(s)), \tilde{\pi}(y, s)) .
$$

Desta forma, obtemos uma definição equivalente para Zhukovskij quase $\tilde{\pi}$-estabilidade.

Observação 4.2.5 Na teoria clássica de sistemas semidinâmicos a estabilidade de Lyapunov implica na estabilidade de Zhukovskij. No entanto, no caso impulsivo podemos encontrar em [19] dois exemplos os quais mostram que cada uma das estabilidades não implica na outra.

Em [19], o autor mostra o seguinte resultado.

Lema 4.2.6 [19, Lema 4.4] Se um ponto $x \in X \backslash M$ é uniformemente assintoticamente Zhukovskij quase $\tilde{\pi}$-stável com $\widetilde{L}^{+}(x) \neq \emptyset$, então $\widetilde{L}^{+}(x)$ é um conjunto minimal.

No próximo teorema, vamos mostrar que se os pontos de um conjunto limite positivo que não estão em $M$ são Zhukovskij quase $\tilde{\pi}$-estáveis então esse conjunto limite é minimal.

Teorema 4.2.7 [14, Teorema 5.1] Sejam $(X, \pi ; M, I)$ um sistema semidinâmico impulsivo e $x \in X$. Suponha que o conjunto não vazio $\widetilde{L}^{+}(x) \backslash M$ seja Zhukovskij quase $\tilde{\pi}$-estável, então $\widetilde{L}^{+}(x)$ é um conjunto minimal.

Demonstração: Seja $y \in \widetilde{L}^{+}(x) \backslash M$ arbitrário. Precisamos mostrar que $\widetilde{L}^{+}(x)=\overline{\widetilde{\pi}^{+}(y)}$. Suponha o contrário, isto é, existe $z \in \widetilde{L}^{+}(x)$ tal que $z \notin \overline{\widetilde{\pi}^{+}(y)}$. Tomemos $\varepsilon=d\left(z, \overline{\tilde{\pi}^{+}(y)}\right)>0$. 
Seja $q \in \tilde{\pi}^{+}(y)$. Note que $\widetilde{\pi}^{+}(y) \subset \widetilde{L}^{+}(x)$, pois $\widetilde{L}^{+}(x) \backslash M$ é positivamente $\tilde{\pi}$-invariante. Por hipótese, existe $\delta=\delta(q)>0$ tal que se $d(v, q)<\delta$, então podemos encontrar uma reparametrização $\tau_{v}$ tal que

$$
d\left(\tilde{\pi}(q, t), \tilde{\pi}\left(v, \tau_{v}(t)\right)\right)<\frac{\varepsilon}{3} \quad \text { para todo } \quad t \geq 0 .
$$

Além disso, existe $s>0$ tal que $d(\tilde{\pi}(x, s), q)<\delta$. Logo, por (4.16) podemos encontrar uma reparametrização $\tau_{w}, w=\tilde{\pi}(x, s)$, tal que

$$
d\left(\widetilde{\pi}(q, t), \tilde{\pi}\left(w, \tau_{w}(t)\right)\right)<\frac{\varepsilon}{3} \quad \text { para todo } \quad t \geq 0
$$

Por outro lado, como $z \in \widetilde{L}^{+}(x)$ então $z \in \widetilde{L}^{+}(w)$. Assim, existe uma sequência $\left\{t_{n}\right\}_{n \geq 1} \subset \mathbb{R}_{+}$ tal que $t_{n} \stackrel{n \rightarrow+\infty}{\longrightarrow}+\infty$ e $\tilde{\pi}\left(w, t_{n}\right) \stackrel{n \rightarrow+\infty}{\longrightarrow} z$. Seja $n_{0} \in \mathbb{N}$ tal que $d\left(z, \tilde{\pi}\left(w, t_{n_{0}}\right)\right)<\frac{\varepsilon}{2}$. Como $\tau_{w}$ é um homeomorfismo, podemos tomar $s_{n}>0$ tal que $t_{n}=\tau_{w}\left(s_{n}\right), n \in \mathbb{N}^{*}$. Então

$$
d\left(\tilde{\pi}\left(q, s_{n_{0}}\right), \tilde{\pi}\left(w, \tau_{w}\left(s_{n_{0}}\right)\right)\right) \geq d\left(z, \tilde{\pi}\left(q, s_{n_{0}}\right)\right)-d\left(z, \tilde{\pi}\left(w, \tau_{w}\left(s_{n_{0}}\right)\right)\right) \geq \varepsilon-\frac{\varepsilon}{2}=\frac{\varepsilon}{2},
$$

contradizendo (4.17). Portanto, $\widetilde{L}^{+}(x)$ é minimal.

Corolário 4.2.8 [14, Corolário 5.1] Sejam $(X, \pi ; M, I)$ um sistema semidinâmico impulsivo e $x \in X$. Suponha que $\widetilde{L}^{+}(x)$ é compacto e $\widetilde{L}^{+}(x) \backslash M \neq \emptyset$.

a) Se $\widetilde{L}^{+}(x) \backslash M$ é Zhukovskij quase $\tilde{\pi}$-estável então todo ponto de $\widetilde{L}^{+}(x) \backslash M$ é $\tilde{\pi}$-recorrente;

b) Se $x \notin M$ e $\widetilde{\pi}^{+}(x)$ é uniformemente assintoticamente Zhukovskij quase $\tilde{\pi}$-estável então todo ponto de $\widetilde{L}^{+}(x) \backslash M$ é $\tilde{\pi}$-recorrente.

Demonstração: a) Pelo Teorema 4.2.7 o conjunto limite positivo $\widetilde{L}^{+}(x)$ é minimal. Pelo Teorema 3.1.3 todo ponto de $\widetilde{L}^{+}(x) \backslash M$ é $\tilde{\pi}$-recorrente.

b) Neste caso, a minimalidade de $\widetilde{L}^{+}(x)$ segue do Lema 4.2.6. O resultado segue pelo Teorema 3.1.3.

Podemos obter também o seguinte resultado.

Teorema 4.2.9 [14, Teorema 5.2] Sejam $(X, \pi ; M, I)$ um sistema semidinâmico impulsivo, $X$ completo e $x \in X \backslash$ M. Suponha que $\widetilde{L}^{+}(x)=\widetilde{\pi}^{+}(x) \cup\left\{x_{k}\right\}_{k \geq 1}$. 
a) Se $\widetilde{L}^{+}(x) \backslash M$ é Zhukovskij quase $\tilde{\pi}$-estável então x é periódico;

b) Se $\tilde{\pi}^{+}(x)$ é uniformemente assintoticamente Zhukovskij quase $\tilde{\pi}$-estável então x é periódico.

Demonstração: $a)$ Note que $\widetilde{L}^{+}(x) \backslash M \neq \emptyset$. Pelo Teorema 4.2.7, o conjunto $\widetilde{L}^{+}(x)$ é minimal. Como $\widetilde{L}^{+}(x)=\widetilde{\pi}^{+}(x) \cup\left\{x_{k}\right\}_{k \geq 1}$, segue do Corolário 3.2.8 que $x$ é periódico.

b) Neste caso, é suficiente usar o Lema 4.2.6 na prova do item $a$ ).

A seguir, apresentamos a definição de atrator uniforme e estabelecemos condições suficientes para que o conjunto limite positivo $\widetilde{L}^{+}(x)$ seja um atrator uniforme.

A próxima definição é estabelecida em [12].

Definição 4.2.10 Sejam $(X, \pi ; M, I)$ um sistema semidinâmico impulsivo e $A$ um subconjunto de $X$. O conjunto

$$
\begin{gathered}
\widetilde{P}_{u}^{+}(A)=\{x \in X: \text { para toda vizinhança } U \text { de } A, \text { existem uma vizinhança } V \text { de } x \\
\text { e } T>0 \text { tais que } \tilde{\pi}(V, t) \subset U \text { para todo } t \geq T\},
\end{gathered}
$$

é chamado de região de atração uniforme de $A$ em relação a $\widetilde{\pi}$. Se $x \in \widetilde{P}_{u}^{+}(A)$, dizemos que $x$ é uniformemente $\tilde{\pi}$-atraído para $A$. Finalmente, diremos que $A$ é um conjunto $\tilde{\pi}$-atrator uniforme se $\widetilde{P}_{u}^{+}(A)$ é uma vizinhança de $A$.

Proposição 4.2.11 [12, Proposição 3.2] Sejam $(X, \pi ; M, I)$ um sistema semidinâmico impulsivo e $A \subset X$ um conjunto compacto. Suponha X localmente compacto. Então

$$
\widetilde{P}_{u}^{+}(A)=\left\{x \in X: \widetilde{J}^{+}(x) \neq \emptyset \text { e } \widetilde{J}^{+}(x) \subset A\right\} .
$$

Os Lemas 4.2.12 e 4.2.13, a seguir, exibem uma relação entre os conjuntos $\widetilde{L}^{+}(x)$ e $\widetilde{J}^{+}(x)$.

Lema 4.2.12 [11, Lema 3.31] Seja $(X, \pi ; M, I)$ um sistema semidinâmico impulsivo. Se $x \notin M e$ $y \in \widetilde{L}^{+}(x)$, então $\widetilde{J}^{+}(x) \subset \widetilde{J}^{+}(y)$. 
Lema 4.2.13 [19, Teorema 4.2] Seja $(X, \pi ; M, I)$ um sistema semidinâmico impulsivo. Se $x \in X \backslash M$ é Zukovskij quase $\tilde{\pi}$-estável, então $\widetilde{L}^{+}(x)=\widetilde{J}^{+}(x)$.

Usando o Lema 4.2.13 e a Proposição 4.2.11, podemos mostrar o seguinte resultado.

Lema 4.2.14 [14, Lema 5.2] Seja $(X, \pi ; M, I)$ um sistema semidinâmico impulsivo e suponha $X$ localmente compacto. Se $x \in X \backslash M$ é Zhukovskij quase $\tilde{\pi}$-estável e $\widetilde{L}^{+}(x)$ é um conjunto não vazio e compacto, então $x \in \widetilde{P}_{u}^{+}\left(\widetilde{L}^{+}(x)\right)$.

Na seguinte proposição, apresentamos condições suficientes para que o conjunto limite positivo esteja contido na sua região de atração uniforme.

Proposição 4.2.15 [14, Proposição 5.1] Seja $(X, \pi ; M, I)$ um sistema semidinâmico impulsivo. Suponha $X$ localmente compacto, $\widetilde{L}^{+}(x) \cap M=\emptyset$ e $\widetilde{L}^{+}(x)$ Zukovskij quase $\widetilde{\pi}$-estável. Se $\widetilde{L}^{+}(x)$ é compacto então $\widetilde{L}^{+}(x) \subset \widetilde{P}_{u}^{+}\left(\widetilde{L}^{+}(x)\right)$.

Demonstração: Como $\widetilde{L}^{+}(x) \cap M=\emptyset$, então $\widetilde{L}^{+}(x)$ é positivamente $\widetilde{\pi}$-invariante, veja o Corolário 1.4.8. Agora, seja $y \in \widetilde{L}^{+}(x)$. Como $\widetilde{L}^{+}(x)$ é positivamente $\widetilde{\pi}$-invariante e fechado, temos $\overline{\widetilde{\pi}^{+}(y)} \subset \widetilde{L}^{+}(x)$. Assim, $\widetilde{L}^{+}(y) \subset \widetilde{L}^{+}(x)$. Note que $\widetilde{L}^{+}(y) \neq \emptyset$, pois $\widetilde{L}^{+}(x)$ é compacto.

Pela hipótese y é Zukovskij quase $\tilde{\pi}$-estável. Logo, pelo Lema 4.2.13, obtemos $\widetilde{L}^{+}(y)=\widetilde{J}^{+}(y)$. Portanto, $\widetilde{J}^{+}(y) \neq \emptyset$ e $\widetilde{J}^{+}(y) \subset \widetilde{L}^{+}(x)$. O resultado segue pela Proposicão 4.2.11.

O último resultado desse capítulo, mostra condições para que o conjunto limite positivo seja $\tilde{\pi}$-atrator uniforme.

Teorema 4.2.16 [14, Teorema 5.3] Seja $(X, \pi ; M, I)$ um sistema semidinâmico impulsivo. Suponha $X$ localmente compacto e $\widetilde{L}^{+}(x) \cap M=\emptyset$ para algum $x \in X$. Se $\widetilde{L}^{+}(x)$ é não vazio, assintoticamente Zhukovskij quase $\tilde{\pi}$-estável e compacto, então $\widetilde{L}^{+}(x)$ é $\tilde{\pi}$-atrator uniforme.

Demonstração: Para cada $y \in \widetilde{L}^{+}(x)$, existe $\delta_{y}>0$ tal que se $d(z, y)<\delta_{y}$ então podemos encontrar uma reparametrização $\tau_{z}$ tal que

$$
d\left(\tilde{\pi}(y, t), \tilde{\pi}\left(z, \tau_{z}(t)\right)\right) \stackrel{t \rightarrow+\infty}{\longrightarrow} 0 .
$$


Como $\widetilde{L}^{+}(x) \cap M=\emptyset$ e $\widetilde{L}^{+}(x)$ é compacto, existe $\beta>0$ tal que $B\left(\widetilde{L}^{+}(x), \beta\right) \cap M=\emptyset$.

Ainda, pela compacidade do conjunto limite, existem $y_{1}, \ldots, y_{n} \in \widetilde{L}^{+}(x)$ tais que $\widetilde{L}^{+}(x) \subset B\left(y_{1}, \frac{\delta_{y_{1}}}{2}\right) \cup \ldots \cup B\left(y_{n}, \frac{\delta_{y_{n}}}{2}\right)$. Tomemos $2 \delta=\min \left\{\delta_{y_{1}}, \ldots, \delta_{y_{n}}, \beta\right\}$.

Afirmamos que $B\left(\widetilde{L}^{+}(x), \delta\right) \subset \widetilde{P}_{u}^{+}\left(\widetilde{L}^{+}(x)\right)$. De fato, seja $w \in B\left(\widetilde{L}^{+}(x), \delta\right)$. Então $w \in B\left(y_{j}, \delta_{y_{j}}\right)$ para algum $j \in\{1, \ldots, n\}$. Assim

$$
d\left(\tilde{\pi}\left(y_{j}, t\right), \tilde{\pi}\left(w, \tau_{w}(t)\right)\right) \stackrel{t \rightarrow+\infty}{\longrightarrow} 0
$$

ou,

$$
d\left(\tilde{\pi}\left(y_{j}, h(t)\right), \tilde{\pi}(w, t)\right) \stackrel{t \rightarrow+\infty}{\longrightarrow} 0
$$

onde $h(t)=\tau_{w}^{-1}(t)$, veja Observação 4.2.4. Como $\widetilde{\pi}^{+}\left(y_{j}\right) \subset \widetilde{L}^{+}(x)$ (pois $\widetilde{L}^{+}(x)$ é positivamente $\tilde{\pi}$-invariante) temos

$$
d\left(\widetilde{L}^{+}(x), \widetilde{\pi}(w, t)\right) \stackrel{t \rightarrow+\infty}{\longrightarrow} 0 .
$$

Então $\widetilde{L}^{+}(w) \neq \emptyset$ e $\widetilde{L}^{+}(w) \subset \widetilde{L}^{+}(x)$. É claro que $\widetilde{J}^{+}(w) \neq \emptyset$. Vamos verificar que $\widetilde{J}^{+}(w) \subset \widetilde{L}^{+}(x)$. De fato, seja $a \in \widetilde{L}^{+}(w)$. Como $w \notin M(\delta<\beta)$ segue pelo Lema 4.2.12 a seguinte inclusão

$$
\widetilde{J}^{+}(w) \subset \widetilde{J}^{+}(a)
$$

Como $a \in \widetilde{L}^{+}(x)$, então $a$ é Zukovskij quase $\widetilde{\pi}$-estável e pelo Lema 4.2.13 temos $\widetilde{J}^{+}(a)=\widetilde{L}^{+}(a)$. Por outro lado, $\widetilde{L}^{+}(x)$ é positivamente $\tilde{\pi}$-invariante. Então $\overline{\widetilde{\pi}^{+}(a)} \subset \widetilde{L}^{+}(x)$, conseq $\tilde{\mathrm{A}} \frac{1}{4}$ entemente, $\widetilde{L}^{+}(a) \subset \widetilde{L}^{+}(x) . \operatorname{Logo}$

$$
\widetilde{J}^{+}(w) \subset \widetilde{L}^{+}(x)
$$

Portanto, pela Proposição 4.2.11 obtemos $w \in \widetilde{P}_{u}^{+}\left(\widetilde{L}^{+}(x)\right)$ e o teorema está provado. 


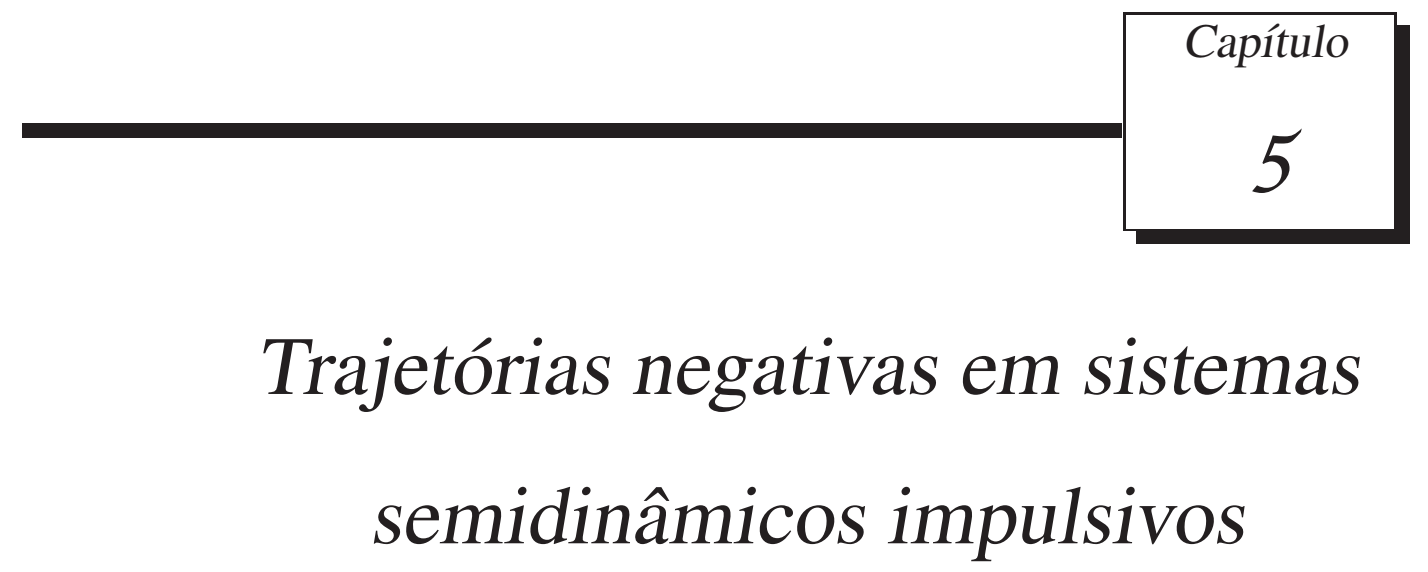

Neste capítulo, apresentamos a noção de semisolução negativa para sistemas semidinâmicos impulsivos. Inicialmente, mostramos como construir uma semisolução negativa para sistemas impulsivos. Na sequência, definimos o conceito de conjunto limite negativo, definimos o conceito para o primeiro prolongamento do conjunto limite negativo e estudamos algumas propriedades topológicas desses conjuntos. Os resultados desse capítulo estão presentes no artigo [15].

\subsection{Trajetória negativa impulsiva}

Na teoria de sistemas semidinâmicos contínuos, é possível estudar o passado da trajetória de um ponto $x \in X$ através das semisoluções negativas que passam por este ponto. Uma função $\sigma_{x}: I_{x} \rightarrow X$ definida em um intervalo $I_{x} \subset \mathbb{R}_{-} \operatorname{com} x \in X$ e $0 \in I_{x}$ é chamada uma semisolução negativa através de $x$ se $\sigma_{x}(0)=x$ e $\pi\left(\sigma_{x}(t), s\right)=\sigma_{x}(t+s)$ quaisquer que sejam $t \in I_{x}$ e $s \in \mathbb{R}_{+}$tais que $t+s \in I_{x}$. O leitor pode consultar [1] e [2] para mais detalhes.

Se $X$ é localmente compacto e $(X, \pi)$ não contém pontos iniciais, então é possível prolongar uma semisolução negativa através de $x \in X$ no intervalo maximal $(-\infty, 0]$, veja [26] por exemplo.

Desta forma, ao longo deste capítulo, vamos assumir que qualquer semisolução negativa no 
sistema semidinâmico contínuo $(X, \pi)$ está definida no intervalo $(-\infty, 0]$.

Dada uma semisolução negativa $\sigma_{x}$ através de um ponto $x \in X$, pretendemos construir uma semisolução negativa impulsiva através de $x$ associada a $\sigma_{x}$. Para isso, precisamos fazer algumas considerações. Primeiro, suponhamos que a função $I: M \rightarrow N$ seja um homeomorfismo com inversa $J: N \rightarrow M$, onde $N=I(M)$. Portanto $N$ é um subconjunto fechado de $X$. Além disso, suponhamos também que exista um número real $\beta>0$ tal que

$$
F(N,(0, \beta)) \cap N=\emptyset \quad \text { e } \quad \pi(N,(0, \beta)) \cap N=\emptyset .
$$

Observação 5.1.1 Pela construção das trajetórias em um sistema semidinâmico impulsivo e pelo fato de $I(M) \cap M=\emptyset$ (veja Hipótese (H2), Seção 1.5 Pag.24), temos que se $x \in M$ então $x$ é um ponto inicial em $(X, \pi ; M, I)$. Então não existe semisolução negativa através de $x$ no sistema impulsivo $(X, \pi ; M, I)$.

Pela Observação 5.1.1, vamos considerar o conjunto $X \backslash M$ e mostraremos que, através de cada ponto $x \in X \backslash M$, existe uma semisolução negativa impulsiva através de $x$ definida em algum intervalo de $\mathbb{R}_{-}$. Em outras palavras, dada uma semisolução negativa $\sigma_{x}$ através de um ponto $x \in X \backslash M$, vamos definir uma função $\widetilde{\sigma}_{x}: I_{x} \rightarrow X$ através do ponto $x, I_{x} \subset(-\infty, 0]$, tal que $\widetilde{\sigma}_{x}(0)=x$ e $\tilde{\pi}\left(\widetilde{\sigma}_{x}(t), s\right)=\widetilde{\sigma}_{x}(t+s)$, quaisquer que sejam $t \in I_{x}$ e $s \in[0,+\infty)$ tais que $t+s \in I_{x}$. Mostraremos que o intervalo maximal de existência $I_{x}$, de $\widetilde{\sigma}_{x}$, é dado por $\left[\alpha_{x}, 0\right]$ (para algum $\left.\alpha_{x}<0\right)$ ou $(-\infty, 0]$.

Dado $x \in X \backslash M$, seja $\sigma_{x}$ uma semisolução negativa através de $x$. A seguir, vamos construir uma semisolução negativa impulsiva $\widetilde{\sigma}_{x}$ através de $x$ associada a $\sigma_{x}$. Para isso vamos considerar dois casos:

Caso 1: $x \notin N$.

Suponhamos que exista $s<0$ tal que $\sigma_{x}(s) \in M, \sigma_{x}((s, 0]) \cap M=\emptyset$ e $\sigma_{x}([s, 0]) \cap N=\emptyset$. Como $\sigma_{x}(s)$ é um ponto inicial em $(X, \pi ; M, I)$, definimos $\widetilde{\sigma}_{x}$ sobre $[s, 0]$ por

$$
\widetilde{\sigma}_{x}(t)=\sigma_{x}(t) .
$$

Neste caso, o intervalo maximal de definição de $\widetilde{\sigma}_{x}$ é dado por $I_{x}=[s, 0]$. 
Suponhamos, agora, que $\sigma_{x}(t) \notin M \cup N$ para todo $t \in(-\infty, 0]$. Neste caso, definimos $\widetilde{\sigma}_{x}$ sobre $I_{x}=(-\infty, 0]$ por

$$
\widetilde{\sigma}_{x}(t)=\sigma_{x}(t) .
$$

Entretanto, se existe $r_{0}<0$ tal que $\sigma_{x}\left(r_{0}\right)=a_{1} \in N$ e $\sigma_{x}(t) \notin N \cup M$ para $t \in\left(r_{0}, 0\right]$, então definimos $\tilde{\sigma}_{x}$ sobre $\left[r_{0}, 0\right]$ por

$$
\widetilde{\sigma}_{x}(t)=\sigma_{x}(t)
$$

Seja $a_{1}^{-}=J\left(a_{1}\right)$. Como $r_{0} \in(-\infty, 0)$, o processo agora continua a partir de $a_{1}^{-} \in M$. Seja $\sigma_{a_{1}^{-}}$uma semisolução negativa contínua através de $a_{1}^{-}$. Temos os seguintes casos:

- Se $\sigma_{a_{1}^{-}}(t) \notin M \cup N$ para todo $t<0$, então definimos $\widetilde{\sigma}_{x}(t)=\sigma_{a_{1}^{-}}\left(t-r_{0}\right)$ para $t \in\left(-\infty, r_{0}\right)$. Assim, $I_{x}=(-\infty, 0]$ e $\widetilde{\sigma}_{x}:(-\infty, 0] \rightarrow X$ é definida por

$$
\tilde{\sigma}_{x}(t)= \begin{cases}\sigma_{x}(t), & r_{0} \leq t \leq 0, \\ \sigma_{a_{1}^{-}}\left(t-r_{0}\right), & -\infty<t<r_{0} .\end{cases}
$$

- Se existe $r_{1}<0$ tal que $\sigma_{a_{1}^{-}}\left(r_{1}\right) \in M, \sigma_{a_{1}^{-}}\left(\left(r_{1}, 0\right)\right) \cap M=\emptyset$ e $\sigma_{a_{1}^{-}}\left(\left[r_{1}, 0\right]\right) \cap N=\emptyset$, então definimos $\widetilde{\sigma}_{x}(t)=\sigma_{a_{1}^{-}}\left(t-r_{0}\right)$ para $t \in\left[r_{0}+r_{1}, r_{0}\right)$. Assim, $I_{x}=\left[r_{0}+r_{1}, 0\right] \operatorname{com} \widetilde{\sigma}_{x}:\left[r_{0}+r_{1}, 0\right] \rightarrow X$ dada por

$$
\tilde{\sigma}_{x}(t)= \begin{cases}\sigma_{x}(t), & r_{0} \leq t \leq 0, \\ \sigma_{a_{1}^{-}}\left(t-r_{0}\right), & r_{0}+r_{1} \leq t<r_{0} .\end{cases}
$$

- Se existe $r_{1}<0$ tal que $\sigma_{a_{1}^{-}}\left(r_{1}\right)=a_{2} \in N$ e $\sigma_{a_{1}^{-}}(t) \notin N \cup M$ para todo $t \in\left(r_{1}, 0\right)$. Então definimos $\widetilde{\sigma}_{x}$ sobre $\left[r_{0}+r_{1}, 0\right]$ por

$$
\tilde{\sigma}_{x}(t)= \begin{cases}\sigma_{x}(t), & r_{0} \leq t \leq 0, \\ \sigma_{a_{1}^{-}}\left(t-r_{0}\right), & r_{0}+r_{1} \leq t<r_{0} .\end{cases}
$$

Definimos $a_{2}^{-}=J\left(a_{2}\right)$, e o processo, neste último caso, continua a partir de $a_{2}^{-}$e assim por diante.

Caso 2: $x \in N$.

Neste caso, denotamos $a_{1}=x$ e $a_{1}^{-}=J(x)$. Seja $\sigma_{a_{1}^{-}}$uma semisolução negativa contínua através 
de $a_{1}^{-}$. Se $\sigma_{a_{1}^{-}}(t) \notin M \cup N$ para todo $t<0$, definimos

$$
\widetilde{\sigma}_{x}(t)= \begin{cases}x, & t=0, \\ \sigma_{a_{1}^{-}}(t), & -\infty<t<0,\end{cases}
$$

e $I_{x}=(-\infty, 0]$

Se existe $r_{0}<0$ tal que $\sigma_{a_{1}^{-}}\left(r_{0}\right) \in M, \sigma_{a_{1}^{-}}\left(\left(r_{0}, 0\right)\right) \cap M=\emptyset$ e $\sigma_{a_{1}^{-}}\left(\left[r_{0}, 0\right]\right) \cap N=\emptyset$, então definimos

$$
\tilde{\sigma}_{x}(t)= \begin{cases}x, & t=0, \\ \sigma_{a_{1}^{-}}(t), & r_{0} \leq t<0,\end{cases}
$$

onde $I_{x}=\left[r_{0}, 0\right]$, pois $\sigma_{a_{1}^{-}}\left(r_{0}\right)$ é um ponto inicial do sistema $(X, \pi ; M, I)$.

Por outro lado, se existe $r_{0}<0$ tal que $\sigma_{a_{1}^{-}}\left(r_{0}\right)=a_{2} \in N$ e $\sigma_{a_{1}^{-}}(t) \notin M \cup N$ para $t \in\left(r_{0}, 0\right)$, então definimos $\widetilde{\sigma}_{x}$ sobre $\left[r_{0}, 0\right]$ por

$$
\tilde{\sigma}_{x}(t)= \begin{cases}x, & t=0, \\ \sigma_{a_{1}^{-}}(t), & r_{0} \leq t<0 .\end{cases}
$$

Definimos agora $a_{2}^{-}=J\left(a_{2}\right)$ e consideremos $\sigma_{a_{2}^{-}}$uma semisolução negativa contínua através de $a_{2}^{-}$. Se $\sigma_{a_{2}^{-}}(t) \notin N \cup M$ para todo $t<0$, definimos

$$
\tilde{\sigma}_{x}(t)= \begin{cases}x, & t=0, \\ \sigma_{a_{1}^{-}}(t), & r_{0} \leq t<0, \\ \sigma_{a_{2}^{-}}\left(t-r_{0}\right), & -\infty<t<r_{0},\end{cases}
$$

e $I_{x}=(-\infty, 0]$

Se existe $r_{1}<0$ tal que $\sigma_{a_{2}^{-}}\left(r_{1}\right) \in M, \sigma_{a_{2}^{-}}\left(\left(r_{1}, 0\right)\right) \cap M=\emptyset$ e $\sigma_{a_{2}^{-}}\left(\left[r_{1}, 0\right]\right) \cap N=\emptyset$, então definimos

$$
\tilde{\sigma}_{x}(t)= \begin{cases}x, & t=0, \\ \sigma_{a_{1}^{-}}(t), & r_{0} \leq t<0, \\ \sigma_{a_{2}^{-}}\left(t-r_{0}\right), & r_{0}+r_{1} \leq t<r_{0} .\end{cases}
$$


Note que $I_{x}=\left[r_{0}+r_{1}, 0\right]$, pois $\sigma_{a_{2}^{-}}\left(r_{1}\right)$ é um ponto inicial em $(X, \pi ; M, I)$.

Entretanto, se existe $r_{1}<0$ tal que $\sigma_{a_{2}^{-}}\left(r_{1}\right)=a_{3} \in N$ e $\sigma_{a_{2}^{-}}(t) \notin N \cup M$ para $t \in\left(r_{0}+r_{1}, r_{0}\right)$, então definimos $\widetilde{\sigma}_{x}$ sobre $\left[r_{0}+r_{1}, 0\right]$ por

$$
\tilde{\sigma}_{x}(t)= \begin{cases}x, & t=0, \\ \sigma_{a_{1}^{-}}(t), & r_{0} \leq t<0, \\ \sigma_{a_{2}^{-}}\left(t-r_{0}\right), & r_{0}+r_{1} \leq t<r_{0} .\end{cases}
$$

Se denotarmos $a_{3}^{-}=J\left(a_{3}\right)$, então o processo, neste último caso, continua a partir de $a_{3}^{-}$e assim por diante.

A seguinte observação descreve a forma geral de uma semisolução negativa impulsiva definida no intervalo $I_{x}=(-\infty, 0]$.

Observação 5.1.2 [15, Observação 4.2] Seja $T_{n}=\sum_{j=0}^{n} r_{j}, n \in \mathbb{N}$. Se $x \notin N$ e $\widetilde{\sigma}_{x}$ é uma semisolução negativa impulsiva através de $x \operatorname{com} I_{x}=(-\infty, 0]$, então ou existem um número $k \in \mathbb{N}$ e semisoluções negativas $\sigma_{a_{1}^{-}}, \ldots, \sigma_{a_{k+1}^{-}}$tais que

$$
\widetilde{\sigma}_{x}(t)= \begin{cases}\sigma_{x}(t), & T_{0} \leq t \leq 0 \\ \sigma_{a_{1}^{-}}\left(t-T_{0}\right), & T_{1} \leq t<T_{0} \\ \sigma_{a_{2}^{-}}\left(t-T_{1}\right), & T_{2} \leq t<T_{1} \\ \vdots & \vdots \\ \sigma_{a_{k}^{-}}\left(t-T_{k-1}\right), & T_{k} \leq t<T_{k-1}, \\ \sigma_{a_{k+1}^{-}}\left(t-T_{k}\right), & t<T_{k}\end{cases}
$$

ou existem semisoluções negativas $\sigma_{a_{1}^{-}}, \sigma_{a_{2}^{-}}, \ldots$, tais que

$$
\tilde{\sigma}_{x}(t)= \begin{cases}\sigma_{x}(t), & T_{0} \leq t \leq 0, \\ \sigma_{a_{n+1}^{-}}\left(t-T_{n}\right), & T_{n+1} \leq t<T_{n}, \quad \text { para todo } n \in \mathbb{N} .\end{cases}
$$

Se $x \in N$ e $\widetilde{\sigma}_{x}$ é uma semisolução negativa impulsiva através de $x \operatorname{com} I_{x}=(-\infty, 0]$, então ou existem um número $k \in \mathbb{N}$ e semisoluções negativas $\sigma_{a_{1}^{-}}, \ldots, \sigma_{a_{k+2}^{-}}$tais que 


$$
\widetilde{\sigma}_{x}(t)= \begin{cases}x, & t=0, \\ \sigma_{a_{1}^{-}}(t), & T_{0} \leq t<0, \\ \sigma_{a_{2}^{-}}\left(t-T_{0}\right), & T_{1} \leq t<T_{0}, \\ \sigma_{a_{3}^{-}}\left(t-T_{1}\right), & T_{2} \leq t<T_{1}, \\ \vdots & \vdots \\ \sigma_{a_{k+1}^{-}}\left(t-T_{k-1}\right), & T_{k} \leq t<T_{k-1}, \\ \sigma_{a_{k+2}^{-}}\left(t-T_{k}\right), & t<T_{k},\end{cases}
$$

ou existem semisoluções negativas $\sigma_{a_{1}^{-}}, \sigma_{a_{2}^{-}}, \ldots$, tais que

$$
\widetilde{\sigma}_{x}(t)= \begin{cases}x, & t=0, \\ \sigma_{x}(t), & T_{0} \leq t<0, \\ \sigma_{a_{n+2}^{-}}\left(t-T_{n}\right), & T_{n+1} \leq t<T_{n}, \quad \text { para todo } n \in \mathbb{N} .\end{cases}
$$

Definição 5.1.3 Sejam $x \in X \backslash M$ e $\tilde{\sigma}_{x}$ uma semisolução negativa impulsiva através de $x$ tal que $I_{x}=(-\infty, 0]$. Dizemos que $x \in J_{k}$ se a semisolução negativa impulsiva $\widetilde{\sigma}_{x}$ é da forma (5.2) ou (5.4). Dizemos que $x \in J_{\infty}$ se a semisolução negativa impulsiva $\widetilde{\sigma}_{x}$ é da forma (5.3) ou (5.5).

Por construção, obtemos que toda semisolução negativa impulsiva é contínua à direita. Além disso, temos o seguinte resultado.

Lema 5.1.4 [15, Lema 4.1] Sejam $(X, \pi ; M, I)$ um sistema semidinâmico impulsivo, $x \in X \backslash M e$ $\tilde{\sigma}_{x}$ uma semisolução negativa impulsiva através de $x$ definida sobre o intervalo $I_{x}$. Então $\widetilde{\sigma}_{x}(0)=x$ e $\tilde{\pi}\left(\widetilde{\sigma}_{x}(t), s\right)=\widetilde{\sigma}_{x}(t+s)$ quaisquer que sejam $t \in I_{x}$ e $s \geq 0$ tais que $t+s \in I_{x}$.

Demonstração: É claro que $\widetilde{\sigma}_{x}(0)=x$. Para a outra igualdade, vamos considerar o caso em que $I_{x}=(-\infty, 0]$ e $x \in J_{\infty}$. É suficiente considerarmos, também, o caso em que $x \notin N$. Sejam $t \leq 0$ e $s \geq 0$ tais que $t+s \leq 0$. Note que se $s=0$, o resultado é válido. Então consideremos $s>0$ e consequentemente $t<0$. Como $x \in J_{\infty}$ e $x \notin N$, existe $n \in \mathbb{N}$ tal que $T_{n} \leq t<T_{n-1}$ (se $n=0$ tomamos $\left.T_{-1}=0\right)$. Podemos escrever $t=T_{n-1}+t^{\prime}$ com $r_{n} \leq t^{\prime}<0$, onde $r_{n}=T_{n}-T_{n-1}$. Pela Observação 5.1.2, podemos escrever

$$
\widetilde{\sigma}_{x}(t)=\sigma_{a_{n}^{-}}\left(t-T_{n-1}\right)=\sigma_{a_{n}^{-}}\left(t^{\prime}\right),
$$


onde denotamos $a_{0}^{-}=x$ e $t=t^{\prime}$ no caso em que $n=0$. Seja $y=\widetilde{\sigma}_{x}(t)$. Como $s>0$, existe $k \in \mathbb{N}$ tal que $s=t_{k}+s^{\prime} \operatorname{com} 0 \leq s^{\prime}<\phi\left(y_{k}^{+}\right)$, onde $t_{0}=0$ e $t_{p+1}=\sum_{j=0}^{p} \phi\left(y_{j}^{+}\right)$para $p=0,1,2, \ldots$

Afirmamos que $y_{1}^{+}=a_{n}$ e $\phi(y)=-t^{\prime}$. De fato, note que

$$
\pi(y, \eta)=\pi\left(\widetilde{\sigma}_{x}(t), \eta\right)=\pi\left(\sigma_{a_{n}^{-}}\left(t^{\prime}\right), \eta\right)=\sigma_{a_{n}^{-}}\left(t^{\prime}+\eta\right) \quad \text { para } \quad 0 \leq \eta<-t^{\prime}
$$

Pela construção de $\widetilde{\sigma}_{x}$, temos $\sigma_{a_{n}^{-}}\left(r_{n}\right)=a_{n+1} \in N, \sigma_{a_{n}^{-}}\left(\left(r_{n}, 0\right]\right) \cap N=\emptyset$ e $\sigma_{a_{n}^{-}}\left(\left[r_{n}, 0\right)\right) \cap M=\emptyset$. Como $t^{\prime}+\eta \in\left[r_{n}, 0\right)$, obtemos o seguinte

$$
\pi\left(y,\left[0,-t^{\prime}\right)\right) \cap M=\emptyset \quad \text { e } \quad \pi\left(y,-t^{\prime}\right)=a_{n}^{-} \in M .
$$

Assim, $\phi(y)=-t^{\prime}$ e $y_{1}^{+}=I\left(y_{1}\right)=I(\pi(y, \phi(y)))=I\left(a_{n}^{-}\right)=I\left(J\left(a_{n}\right)\right)=a_{n}$. Seguindo o mesmo raciocínio, podemos concluir que:

$$
\begin{gathered}
y_{1}^{+}=a_{n}, y_{2}^{+}=a_{n-1}, \ldots, y_{k}^{+}=a_{n-k+1}, \\
\phi(y)=-t^{\prime}, \phi\left(y_{1}^{+}\right)=-r_{n-1}, \ldots, \phi\left(y_{k}^{+}\right)=-r_{n-k} .
\end{gathered}
$$

Então

$$
s=t_{k}+s^{\prime}=\sum_{j=0}^{k-1} \phi\left(y_{j}^{+}\right)+s^{\prime}=-t^{\prime}-r_{n-1}-\ldots-r_{n-k+1}+s^{\prime} .
$$

Logo,

$$
t+s=T_{n-1}+t^{\prime}+s=r_{0}+r_{1}+\ldots+r_{n-k}+s^{\prime}=T_{n-k}+s^{\prime}
$$

$T_{n-k} \leq t+s$ e $t+s=T_{n-k}+s^{\prime}=T_{n-k-1}+r_{n-k}+s^{\prime}<T_{n-k-1}$. Portanto,

$$
\begin{gathered}
\tilde{\pi}\left(\widetilde{\sigma}_{x}(t), s\right)=\tilde{\pi}(y, s)=\pi\left(y_{k}^{+}, s^{\prime}\right)=\pi\left(a_{n-k+1}, s^{\prime}\right)= \\
=\pi\left(\sigma_{a_{n-k}^{-}}\left(r_{n-k}\right), s^{\prime}\right)=\sigma_{a_{n-k}^{-}}\left(r_{n-k}+s^{\prime}\right)=\sigma_{a_{n-k}^{-}}\left(T_{n-k}-T_{n-k-1}+s^{\prime}\right)= \\
=\sigma_{a_{n-k}^{-}}\left(\left(T_{n-k}+s^{\prime}\right)-T_{n-k-1}\right)=\sigma_{a_{n-k}^{-}}\left(t+s-T_{n-k-1}\right)=\widetilde{\sigma}_{x}(t+s) .
\end{gathered}
$$

A prova está completa. 
Definição 5.1.5 Dado um ponto $x \in X \backslash M$ e uma semisolução negativa impulsiva $\widetilde{\sigma}_{x}$ através de $x$, definida em $I_{x}$, definimos uma órbita de $x$ em relação a $\widetilde{\sigma}_{x}$ por

$$
\widetilde{\pi}_{\widetilde{\sigma}_{x}}(x)=\widetilde{\sigma}_{x}\left(I_{x}\right) \cup \tilde{\pi}^{+}(x) .
$$

Vamos denotar $\widetilde{\pi}_{\widetilde{\sigma}_{x}}(x)$ simplesmente por $\widetilde{\pi}_{\sigma}(x)$.

\subsection{Convergência para semisoluções negativas impulsivas}

Seja $(X, \pi ; M, I)$ um sistema semidinâmico impulsivo. Consideremos $x \in X \backslash M, \widetilde{\sigma}_{x}$ uma semisolução negativa impulsiva através de $x$ definida em $I_{x}$ e $\left\{z_{n}\right\}_{n \geq 1}$ uma sequência em $X$ tal que $z_{n} \stackrel{n \rightarrow+\infty}{\longrightarrow} x$. Uma pergunta natural que surge é se existe alguma semisolução negativa impulsiva $\widetilde{\sigma}_{n}$ através de $z_{n}, n \in \mathbb{N}^{*}$, tal que $\widetilde{\sigma}_{n}(t) \stackrel{n \rightarrow+\infty}{\longrightarrow} \widetilde{\sigma}_{x}(t)$ para cada $t \in I_{x}$. Mas esta convergência não vale em geral. Devido aos efeitos de impulsos, vamos mostrar que para cada $t \in I_{x}, x \notin N$, existe uma sequência $\left\{\varepsilon_{n}\right\}_{n \geq 1} \subset \mathbb{R}$ tal que $\varepsilon_{n} \stackrel{n \rightarrow+\infty}{\longrightarrow} 0$ e $\widetilde{\sigma}_{n}\left(t+\varepsilon_{n}\right) \stackrel{n \rightarrow+\infty}{\longrightarrow} \widetilde{\sigma}_{x}(t)$.

Dados $x \in X$ e $\sigma_{x}$ uma semisolução negativa através de $x$, definimos a função $\psi\left(\sigma_{x}, x\right)$ por

$$
\psi\left(\sigma_{x}, x\right):=\psi_{\sigma}(x)= \begin{cases}r, & \text { se } \sigma_{x}(r) \in N \text { e } \sigma_{x}(t) \notin N \text { para } r<t<0 \\ -\infty, & \text { se } \sigma_{x}(t) \notin N \text { para todo } t<0\end{cases}
$$

Se $\sigma_{x}((-\infty, 0)) \cap N \neq \emptyset$, então a função $\psi\left(\sigma_{x}, x\right)$ representa o maior tempo negativo para o qual a semisolução negativa $\sigma_{x}$ encontra o conjunto $N$. A seguir, discutimos a continuidade, em algum sentido, da função $\psi_{\sigma}$.

Da mesma forma que definimos para o conjunto $M$ no Capítulo 1 , diremos que o conjunto $N$ satisfaz a condição forte de tubo e escrevemos (STC) se para cada ponto $x \in N$ existe um $\lambda$-tubo $F(L,[0,2 \lambda])$ dado por uma seção $S=F(L, \lambda)$ através de $x$ tal que $S=N \cap F(L,[0,2 \lambda])$.

Definição 5.2.1 Um ponto $x \in X$ satisfaz a condição de convergência (condição $C$ ) se para qualquer sequência $\left\{z_{n}\right\}_{n \geq 1} \subset X$ tal que $z_{n} \stackrel{n \rightarrow+\infty}{\longrightarrow} x$ e para qualquer semisolução negativa através de $x$, $\sigma_{x}$, existe uma semisolução negativa $\sigma_{n}$ através de $z_{n}, n \in \mathbb{N}^{*}$, tal que $\sup d\left(\sigma_{n}(t), \sigma_{x}(t)\right) \stackrel{n \rightarrow+\infty}{\longrightarrow} 0$.

O Teorema 5.2.2 exibe um resultado de "continuidade" para a função $\psi_{\sigma}$. 
Teorema 5.2.2 [15, Teorema 4.1] Sejam $(X, \pi ; M, I)$ um sistema semidinâmico impulsivo e $x \in$ $X \backslash N$. Suponha que $N$ satisfaz a condição STC e que $x$ satisfaz a condição $C$. Sejam $\sigma_{x}$ uma semisolução negativa através de $x$ e $\left\{z_{n}\right\}_{n \geq 1} \subset X$ uma sequência tal que $z_{n} \stackrel{n \rightarrow+\infty}{\longrightarrow} x$. Então para cada $n \in \mathbb{N}^{*}$, existe uma semisolução negativa $\sigma_{n}$ através de $z_{n}$ tal que $\sigma_{n}(t) \stackrel{n \rightarrow+\infty}{\longrightarrow} \sigma_{x}(t)$ para todo $t \leq 0 e \psi_{\sigma_{n}}\left(z_{n}\right) \stackrel{n \rightarrow+\infty}{\longrightarrow} \psi_{\sigma}(x)$.

Demonstração: Como $x$ satisfaz a condição $C$, existe uma semisolução negativa $\sigma_{n}$ através de $z_{n}$, $n \in \mathbb{N}^{*}$, tal que $\sup _{t \leq 0} d\left(\sigma_{n}(t), \sigma_{x}(t)\right) \stackrel{n \rightarrow+\infty}{\longrightarrow} 0$.

É suficiente considerarmos o caso em que $\psi_{\sigma}(x)=u \in(-\infty, 0)$. Isto implica que $\sigma_{x}(u) \in N$ e $\sigma_{x}(t) \notin N$ para $t \in(u, 0)$. Como $N$ satisfaz a condição STC, existe um STC-tubo $F(L,[0,2 \lambda])$ através de $\sigma_{x}(u)$ dado por uma seção $S \subset N$ tal que $S=N \cap F(L,[0,2 \lambda])$. Além disso, o tubo é uma vizinhança de $\sigma_{x}(u)$, logo existe $\xi>0$ tal que

$$
B\left(\sigma_{x}(u), \xi\right) \subset F(L,[0,2 \lambda]) .
$$

Como $\sigma_{n}(u) \stackrel{n \rightarrow+\infty}{\longrightarrow} \sigma_{x}(u)$, existe um número natural $n_{0} \in \mathbb{N}$ tal que $\sigma_{n}(u) \in B\left(\sigma_{x}(u), \xi\right)$ para todo $n>n_{0}$. Pelas propriedades de tubo, existe uma sequência $\left\{\varepsilon_{n}\right\}_{n \geq 1} \subset \mathbb{R}, \varepsilon_{n} \stackrel{n \rightarrow+\infty}{\longrightarrow} 0$, tal que $\sigma_{n}\left(u+\varepsilon_{n}\right) \in S \cap B\left(\sigma_{x}(u), \xi\right)$ para todo $n>n_{0}$.

Afirmamos que $\psi_{\sigma_{n}}\left(z_{n}\right)=\varepsilon_{n}+u$ para todo $n>n_{0}$. Suponhamos que isso não ocorra. Então podemos assumir que existe $\eta_{n} \in\left(\varepsilon_{n}+u, 0\right)$ tal que $\sigma_{n}\left(\eta_{n}\right) \in N, n>n_{0}$. Note que $\left\{\eta_{n}\right\}_{n>n_{0}}$ admite uma subsequência convergente, ou seja,

$$
\eta_{n_{k}} \stackrel{k \rightarrow+\infty}{\longrightarrow} a \in[u, 0]
$$

Assim, pela continuidade de $\sigma_{x}$ e pela condição $\mathrm{C}$, obtemos

$$
\begin{aligned}
d\left(\sigma_{n_{k}}\left(\eta_{n_{k}}\right), \sigma_{x}(a)\right) & \leq d\left(\sigma_{n_{k}}\left(\eta_{n_{k}}\right), \sigma_{x}\left(\eta_{n_{k}}\right)\right)+d\left(\sigma_{x}\left(\eta_{n_{k}}\right), \sigma_{x}(a)\right) \\
& \leq \sup _{t \leq 0} d\left(\sigma_{n_{k}}(t), \sigma_{x}(t)\right)+d\left(\sigma_{x}\left(\eta_{n_{k}}\right), \sigma_{x}(a)\right) \stackrel{k \rightarrow+\infty}{\longrightarrow} 0 .
\end{aligned}
$$

Caso 1: $a=u$.

Neste caso, usando (5.6), existe $m_{0}>n_{0}$ tal que $\sigma_{n_{k}}\left(\eta_{n_{k}}\right) \in B\left(\sigma_{x}(u), \xi\right)$ para $n_{k}>m_{0}$. Como $S=N \cap F(L,[0,2 \lambda])$ temos $\sigma_{n_{k}}\left(\eta_{n_{k}}\right) \in S \cap B\left(\sigma_{x}(u), \xi\right)$ para $n_{k}>m_{0}$. Por outro lado, pela proprie- 
dade do conjunto $N$, existe $\beta>0$ tal que

$$
F\left(\sigma_{n_{k}}\left(\eta_{n_{k}}\right),(0, \beta)\right) \cap N=\emptyset \quad \text { e } \quad \pi\left(\sigma_{n_{k}}\left(\eta_{n_{k}}\right),(0, \beta)\right) \cap N=\emptyset
$$

para todo $n_{k}>m_{0}$. Tomemos $n_{k_{0}}>m_{0}$ tal que $u+\varepsilon_{n_{k_{0}}}<0$ e $0<\eta_{n_{k_{0}}}-u-\varepsilon_{n_{k_{0}}}<\beta$. Então $\sigma_{n_{k_{0}}}\left(u+\varepsilon_{n_{k_{0}}}\right) \in F\left(\sigma_{n_{k_{0}}}\left(\eta_{n_{k_{0}}}\right),(0, \beta)\right) \cap N$ contradizendo (5.7).

Caso 2: $u<a \leq 0$.

Como $\left\{\sigma_{n_{k}}\left(\eta_{n_{k}}\right)\right\}_{k \geq 1} \subset N$ e $N$ é fechado, temos $\sigma_{x}(a) \in N$ que é uma contradição, pois $\psi_{\sigma}(x)=u$.

Desta forma concluímos que $\psi_{\sigma_{n}}\left(z_{n}\right)=\varepsilon_{n}+u$ para todo $n>n_{0}$. Portanto, $\psi_{\sigma_{n}}\left(z_{n}\right) \stackrel{n \rightarrow+\infty}{\longrightarrow} u$.

A seguir, apresentamos o resultado de convergência.

Teorema 5.2.3 [15, Teorema 4.2] Seja $(X, \pi ; M, I)$ um sistema semidinâmico impulsivo. Suponha que $N$ satisfaz a condição STC e que cada ponto de $X \backslash N$ satisfaz a condição $C$. Sejam $x \in$ $X \backslash(M \cup N)$, $\widetilde{\sigma}_{x}$ é uma semisolução negativa impulsiva através de $x$ definida no intervalo $(-\infty, 0]$ $e\left\{z_{n}\right\}_{n \geq 1}$ é uma sequência em $X$ tal que $z_{n} \stackrel{n \rightarrow+\infty}{\longrightarrow} x$. Então, dado $t \leq 0$, existem uma sequência $\left\{\varepsilon_{n}\right\}_{n \geq 1} \subset \mathbb{R}$ tal que $\varepsilon_{n} \stackrel{n \rightarrow+\infty}{\longrightarrow} 0$ e uma semisolução negativa impulsiva $\widetilde{\sigma}_{n}$ através de $z_{n}, n \in \mathbb{N}^{*}$, definida no intervalo $(-\infty, 0]$ tal que $\widetilde{\sigma}_{n}\left(t+\varepsilon_{n}\right) \stackrel{n \rightarrow+\infty}{\longrightarrow} \widetilde{\sigma}_{x}(t)$.

Demonstração: Vamos supor que $x \in J_{\infty}$. Como $x \notin N$, segue pela Observação 5.1.2, igualdade (5.3), que existem semisoluções negativas $\sigma_{a_{1}^{-}}, \sigma_{a_{2}^{-}}, \ldots$, tais que

$$
\tilde{\sigma}_{x}(t)= \begin{cases}\sigma_{x}(t), & T_{0} \leq t \leq 0, \\ \sigma_{a_{n+1}^{-}}\left(t-T_{n}\right), & T_{n+1} \leq t<T_{n}, \quad \text { para todo } n \in \mathbb{N} .\end{cases}
$$

Lembremos que $T_{n}=\sum_{j=0}^{n} r_{j}, n \in \mathbb{N}$.

Como $z_{n} \stackrel{n \rightarrow+\infty}{\longrightarrow} x$ e $x \notin N$, podemos assumir, sem perda de generalidade, que $z_{n} \notin N$ para todo $n \in \mathbb{N}^{*}$

Pela hipótese e pelo Teorema 5.2.2, existe uma semisolução negativa $\sigma_{n}$ através de $z_{n}$ definida 
no intervalo $(-\infty, 0]$ tal que $\sigma_{n}(t) \stackrel{n \rightarrow+\infty}{\longrightarrow} \sigma_{x}(t), t \leq 0$, e $\psi_{\sigma_{n}}\left(z_{n}\right) \stackrel{n \rightarrow+\infty}{\longrightarrow} \psi_{\sigma}(x)$. Pela continuidade da função $J$ obtemos

$$
\left(a_{n}\right)_{1}^{-}=J\left(\left(a_{n}\right)_{1}\right) \stackrel{n \rightarrow+\infty}{\longrightarrow} J\left(a_{1}\right)=a_{1}^{-}
$$

onde $a_{1}=\sigma_{x}\left(\psi_{\sigma}(x)\right)$ e $\left(a_{n}\right)_{1}=\sigma_{n}\left(\psi_{\sigma_{n}}\left(z_{n}\right)\right), n=1,2, \ldots$. Novamente, usando a hipótese desse teorema e pelo Teorema 5.2.2, existe uma semisolução negativa $\sigma_{n_{1}}$ através de $\left(a_{n}\right)_{1}^{-}$tal que $\sigma_{n_{1}}(t) \stackrel{n \rightarrow+\infty}{\longrightarrow} \sigma_{a_{1}^{-}}(t), t \leq 0, \mathrm{e}$

$$
\psi_{\sigma_{n_{1}}}\left(\left(a_{n}\right)_{1}^{-}\right) \stackrel{n \rightarrow+\infty}{\longrightarrow} \psi_{\sigma_{a_{1}^{-}}}\left(a_{1}^{-}\right)
$$

Continuamos com este processso e obtemos uma semisolução negativa impulsiva através de $z_{n}$ dada por

$$
\widetilde{\sigma}_{n}(t)= \begin{cases}\sigma_{n}(t), & T_{0}^{n} \leq t \leq 0 \\ \sigma_{n_{k+1}}\left(t-T_{k}^{n}\right), & T_{k+1}^{n} \leq t<T_{k}^{n}, \quad k \in \mathbb{N}\end{cases}
$$

onde $T_{k}^{n}=\sum_{j=0}^{k} r_{j}^{n}, k \in \mathbb{N}$ e $n \in \mathbb{N}^{*}\left(r_{0}^{n}=\psi_{\sigma_{n}}\left(z_{n}\right)\right.$ e $\left.r_{j}^{n}=\psi_{\sigma_{n_{j}}}\left(\left(a_{n}\right)_{j}^{-}\right), j=1,2, \ldots\right)$. Por construção, temos $r_{j}^{n} \stackrel{n \rightarrow+\infty}{\longrightarrow} r_{j}$ para cada $j \in \mathbb{N}^{*}$. Então $T_{k}^{n} \stackrel{n \rightarrow+\infty}{\longrightarrow} T_{k}$, para todo $k \in \mathbb{N}$.

Se $t=0$, basta tomarmos $\varepsilon_{n}=0$ para todo $n$, e a convergência segue.

Se $t<0$, existe $k \in \mathbb{N}$ tal que $T_{k} \leq t<T_{k-1}$, onde consideramos $T_{-1}=0$ se $k=0$. Podemos escrever $t=T_{k-1}+t^{\prime}$ onde $r_{k} \leq t^{\prime}<0$ e $r_{k}=T_{k}-T_{k-1}$. Além disso,

$$
\widetilde{\sigma}_{x}(t)=\sigma_{a_{k}^{-}}\left(t-T_{k-1}\right)=\sigma_{a_{k}^{-}}\left(t^{\prime}\right)
$$

No caso em que $k=0$ consideramos $a_{0}^{-}=x$ e $t=t^{\prime}$. Temos dois casos para analisar:

Caso 1: $T_{k}<t<T_{k-1}$.

Como $T_{k}^{n} \stackrel{n \rightarrow+\infty}{\longrightarrow} T_{k}$, existe $m_{0}>0$ tal que $T_{k}^{n}<t<T_{k-1}^{n}$ para todo $n>m_{0}$. Daí, existe $t_{n}^{\prime} \in\left[r_{k}^{n}, 0\right)$, $r_{k}^{n}=T_{k}^{n}-T_{k-1}^{n}$, tal que $t=T_{k-1}^{n}+t_{n}^{\prime}$, para $n>m_{0}$, satisfazendo

$$
t_{n}^{\prime} \stackrel{n \rightarrow+\infty}{\longrightarrow} t^{\prime}
$$

Notemos que $\widetilde{\sigma}_{n}(t)=\sigma_{n_{k}}\left(t-T_{k-1}^{n}\right)=\sigma_{n_{k}}\left(t_{n}^{\prime}\right)$ para $n>m_{0}$. Tomemos $\varepsilon_{n}=T_{k-1}^{n}+t^{\prime}-t, n \in \mathbb{N}^{*}$. 
Note que $\varepsilon_{n} \stackrel{n \rightarrow+\infty}{\longrightarrow} 0$. Então para $n$ suficientemente grande, temos

$$
T_{k}^{n}<t+\varepsilon_{n}<T_{k-1}^{n} \Longleftrightarrow T_{k}^{n}<T_{k-1}^{n}+t^{\prime}<T_{k-1}^{n}
$$

Portanto, obtemos

$$
\widetilde{\sigma}_{n}\left(t+\varepsilon_{n}\right)=\widetilde{\sigma}_{n}\left(T_{k-1}^{n}+t^{\prime}\right)=\sigma_{n_{k}}\left(T_{k-1}^{n}+t^{\prime}-T_{k-1}^{n}\right)=\sigma_{n_{k}}\left(t^{\prime}\right) \stackrel{n \rightarrow+\infty}{\longrightarrow} \sigma_{a_{k}^{-}}\left(t^{\prime}\right)=\widetilde{\sigma}_{x}(t) .
$$

Caso 2: $t=T_{k}$.

Neste caso, para cada $n \in \mathbb{N}^{*}$, existe $u_{k}^{n}$ tal que $t=T_{k}^{n}+u_{k}^{n}$ e $u_{k}^{n} \stackrel{n \rightarrow+\infty}{\longrightarrow} 0$.

Escolha $\varepsilon_{n}=-u_{k}^{n}, n \in \mathbb{N}^{*}$. Então, $\varepsilon_{n} \stackrel{n \rightarrow+\infty}{\longrightarrow} 0 \mathrm{e}$

$$
\widetilde{\sigma}_{n}\left(t+\varepsilon_{n}\right)=\widetilde{\sigma}_{n}\left(T_{k}^{n}\right)=\sigma_{n_{k}}\left(r_{k}^{n}\right) \stackrel{n \rightarrow+\infty}{\longrightarrow} \sigma_{a_{k}^{-}}\left(r_{k}\right)=\widetilde{\sigma}_{x}\left(T_{k}\right)=\widetilde{\sigma}_{x}(t)
$$

\subsection{Invariância e conjuntos limite}

Nesta seção, extendemos o conceito de invariância negativa para sistemas impulsivos. A teoria de invariância negativa para o caso contínuo pode ser encontrada em [1] e [2].

Definição 5.3.1 Um subconjunto não vazio $A$ de $X$ é chamado de:

i) negativamente fortemente invariante se $\widetilde{\sigma}_{x}\left(I_{x}\right) \subset A$ para qualquer $x \in A \backslash M$ e para qualquer semisolução negativa impulsiva $\widetilde{\sigma}_{x}$ através de $x$, onde $I_{x}$ é o intervalo maximal de definição de $\widetilde{\sigma}_{x}$

ii) negativamente fracamente invariante se para cada $x \in A \backslash M$ existe uma semisolução negativa impulsiva $\widetilde{\sigma}_{x}$ através de $x$ tal que $\widetilde{\sigma}_{x}\left(I_{x}\right) \subset A$, onde $I_{x}$ é o intervalo maximal de definição de $\widetilde{\sigma}_{x}$;

iii) fortemente (fracamente) invariante se este é positivamente $\widetilde{\pi}$-invariante e negativamente fortemente (fracamente) invariante. 
Lema 5.3.2 [15, Lema 4.2] Sejam $(X, \pi ; M, I)$ um sistema semidinâmico impulsivo e $x \in X \backslash M$. O conjunto $\widetilde{\sigma}_{x}\left(I_{x}\right)$ é negativamente fracamente invariante para qualquer semisolução negativa impulsiva $\widetilde{\sigma}_{x}$ através de $x$. Consequentemente, $\tilde{\pi}_{\sigma}(x)$ é fracamente invariante.

Demonstração: Pela construção de uma semisolução negativa impulsiva através de $x$, temos que $I_{x}=\left[\alpha_{x}, 0\right]$ ou $I_{x}=(-\infty, 0]$. Suponhamos, inicialmente, que $I_{x}=\left[\alpha_{x}, 0\right]$. Dado $y \in \tilde{\sigma}_{x}\left(I_{x}\right) \backslash M$, existe $s \in I_{x}$ tal que $y=\widetilde{\sigma}_{x}(s)$. Como $y \notin M$, é suficiente definirmos $\widetilde{\sigma}_{y}(t)=\widetilde{\sigma}_{x}(t+s)$ para todo $t \in\left[\alpha_{x}-s, 0\right]$. Agora, se $I_{x}=(-\infty, 0]$, é suficiente definirmos $\widetilde{\sigma}_{y}(t)=\widetilde{\sigma}_{x}(t+s)$ para todo $t \leq 0$.

No que segue, apresentamos a definição do conjunto limite negativo de um ponto $x \in X \backslash M$.

Definição 5.3.3 Sejam $x \in X \backslash M$ e $\widetilde{\sigma}_{x}$ uma semisolução negativa impulsiva através de $x$ definida no intervalo $(-\infty, 0]$. O conjunto limite negativo de $x$ em relação a $\widetilde{\sigma}_{x}$ é dado por $\widetilde{L}_{\widetilde{\sigma}_{x}}^{-}(x)=$ $\bigcap_{t \leq 0} \overline{\widetilde{\sigma}_{x}((-\infty, t])}$. Vamos denotar $\widetilde{L}_{\widetilde{\sigma}_{x}}^{-}(x)$ simplesmente por $\widetilde{L}_{\sigma}^{-}(x)$.

O Lema 5.3.4 caracteriza o conjunto limite negativo de um ponto $x \in X \backslash M$.

Lema 5.3.4 [15, Lema 4.3] Sejam $x \in X \backslash M$ e $\widetilde{\sigma}_{x}$ uma semisolução negativa impulsiva através de $x$ definida no intervalo $(-\infty, 0]$. Um ponto $y \in X$ pertence ao conjunto $\widetilde{L}_{\sigma}^{-}(x)$ se, e somente se, existe uma sequência $\left\{t_{n}\right\}_{n \geq 1} \subset \mathbb{R}_{-}$tal que $t_{n} \stackrel{n \rightarrow+\infty}{\longrightarrow}-\infty e \widetilde{\sigma}_{x}\left(t_{n}\right) \stackrel{n \rightarrow+\infty}{\longrightarrow} y$.

O próximo teorema mostra um resultado sobre invariância positiva.

Teorema 5.3.5 [15, Teorema 4.3] Sejam $(X, \pi ; M, I)$ um sistema semidinâmico impulsivo, $x \in X \backslash$ Me $\tilde{\sigma}_{x}$ uma semisolução negativa impulsiva através de $x$ definida no intervalo $(-\infty, 0]$. O conjunto $\widetilde{L}_{\sigma}^{-}(x)$ é fechado e $\widetilde{L}_{\sigma}^{-}(x) \backslash M$ é positivamente $\tilde{\pi}$-invariante.

Demonstração: Pela Definição 5.3 .3 o conjunto $\widetilde{L}_{\sigma}^{-}(x)$ é fechado. Vamos mostrar que $\widetilde{L}_{\sigma}^{-}(x) \backslash M$ é positivamente $\tilde{\pi}$-invariante. De fato, sejam $y \in \widetilde{L}_{\sigma}^{-}(x) \backslash M$ e $s \geq 0$. Então existe uma sequência $\left\{t_{n}\right\}_{n \geq 1} \subset \mathbb{R}_{-}$tal que $t_{n} \stackrel{n \rightarrow+\infty}{\longrightarrow}-\infty \mathrm{e}$

$$
\widetilde{\sigma}_{x}\left(t_{n}\right) \stackrel{n \rightarrow+\infty}{\longrightarrow} y
$$


Pelo Lema 1.4.5, existe uma sequência $\left\{\varepsilon_{n}\right\}_{n \geq 1} \subset \mathbb{R}$ tal que $\varepsilon_{n} \stackrel{n \rightarrow+\infty}{\longrightarrow} 0 \mathrm{e}$

$$
\tilde{\pi}\left(\widetilde{\sigma}_{x}\left(t_{n}\right), s+\varepsilon_{n}\right) \stackrel{n \rightarrow+\infty}{\longrightarrow} \tilde{\pi}(y, s) .
$$

Como $t_{n}+s+\varepsilon_{n}<0$ para $n$ suficientemente grande, temos

$$
\widetilde{\sigma}_{x}\left(t_{n}+s+\varepsilon_{n}\right)=\tilde{\pi}\left(\widetilde{\sigma}_{x}\left(t_{n}\right), s+\varepsilon_{n}\right) \stackrel{n \rightarrow+\infty}{\longrightarrow} \tilde{\pi}(y, s) .
$$

Então $\tilde{\pi}(y, s) \in \widetilde{L}_{\sigma}^{-}(x) \backslash M$, pois $t_{n}+s+\varepsilon_{n} \stackrel{n \rightarrow+\infty}{\longrightarrow}-\infty$ e $I(M) \cap M=\emptyset$. Como $s$ é arbitrário, o resultado segue.

O Teorema 5.3.6, abaixo, lida com a invariância negativa fraca do conjunto limite negativo.

Teorema 5.3.6 [15, Teorema 4.4] Seja $(X, \pi ; M, I)$ um sistema semidinâmico impulsivo. Sejam $\gamma>0$ tal que $\pi(M,[0, \gamma]) \cap N=\emptyset, x \in X \backslash M$ e $\widetilde{\sigma}_{x}$ uma semisolução negativa impulsiva através de

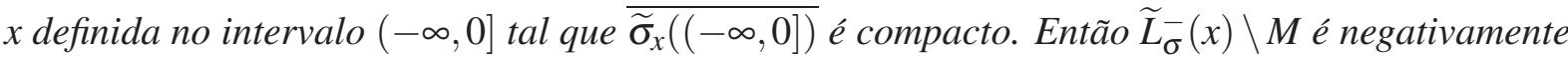
fracamente invariante.

Demonstração: Seja $y \in \widetilde{L}_{\sigma}^{-}(x) \backslash M$. Então existe uma sequência $\left\{t_{n}\right\}_{n \geq 1} \subset \mathbb{R}_{-}$tal que $t_{n} \stackrel{n \rightarrow+\infty}{\longrightarrow}-\infty$ e $\widetilde{\sigma}_{x}\left(t_{n}\right) \stackrel{n \rightarrow+\infty}{\longrightarrow} y$. Seja $s \geq 0$ fixo e arbitrário. Defina $z_{n}=\widetilde{\sigma}_{x}\left(t_{n}-s\right), n=1,2, \ldots$. Pela compacidade de $\overline{\widetilde{\sigma}_{x}((-\infty, 0])}$, podemos supor, sem perda de generalidade, que

$$
z_{n} \stackrel{n \rightarrow+\infty}{\longrightarrow} w \in \widetilde{L}_{\sigma}^{-}(x)
$$

Usando o Lema 1.4.6, obtemos

$$
\widetilde{\sigma}_{x}\left(t_{n}+\eta\right)=\tilde{\pi}\left(\widetilde{\sigma}_{x}\left(t_{n}\right), \eta\right) \stackrel{n \rightarrow+\infty}{\longrightarrow} \tilde{\pi}(y, \eta)
$$

para todo $\eta \in[0, \phi(y))$. Seja $\left\{\eta_{\ell}\right\}_{\ell \geq 1} \subset \mathbb{R}_{+}$uma sequência não crescente de números positivos tal que $\eta_{\ell} \stackrel{\ell \rightarrow+\infty}{\longrightarrow} 0$. Podemos assumir que existem números $k, p \in \mathbb{N}$ tais que

$$
\sum_{j=-1}^{k-1} \phi\left(w_{j}^{+}\right)<s+\eta_{\ell}<\sum_{j=-1}^{k} \phi\left(w_{j}^{+}\right) \quad \text { e } \quad \sum_{j=-1}^{p-1} \phi\left(I(w)_{j}^{+}\right)<s+\eta_{\ell}<\sum_{j=-1}^{p} \phi\left(I(w)_{j}^{+}\right)
$$

para todo $\ell=1,2, \ldots$, onde $\phi\left(w_{-1}^{+}\right)=\phi\left(I(w)_{-1}^{+}\right)=0$. Vamos considerar os seguintes casos: 
Caso 1: $w \notin M$.

Neste caso, usando o Lema 1.4.6 e (5.9) obtemos

$$
\tilde{\pi}\left(y, \eta_{\ell}\right) \stackrel{n \rightarrow+\infty}{\longleftarrow} \widetilde{\sigma}_{x}\left(t_{n}+\eta_{\ell}\right)=\tilde{\pi}\left(\widetilde{\sigma}_{x}\left(t_{n}-s\right), s+\eta_{\ell}\right)=\tilde{\pi}\left(z_{n}, s+\eta_{\ell}\right) \stackrel{n \rightarrow+\infty}{\longrightarrow} \tilde{\pi}\left(w, s+\eta_{\ell}\right) .
$$

Assim, $\tilde{\pi}\left(w, s+\eta_{\ell}\right)=\tilde{\pi}\left(y, \eta_{\ell}\right)$ para todo $\ell=1,2, \ldots$ Como $\tilde{\pi}_{x}$ é contínua à direita, obtemos $\tilde{\pi}(w, s)=y$ quando $\eta_{\ell} \stackrel{\ell \rightarrow+\infty}{\longrightarrow} 0$.

Por outro lado, como $\widetilde{L}_{\sigma}^{-}(x) \backslash M$ é positivamente $\tilde{\pi}$-invariante, temos que $\widetilde{\pi}^{+}(w) \subset \widetilde{L}_{\sigma}^{-}(x)$. Então

$$
\tilde{\pi}(w,[0, s]) \subset \widetilde{L}_{\sigma}^{-}(x) \quad \text { e } \quad \tilde{\pi}(w, s)=y .
$$

Logo, podemos definir $\widetilde{\sigma}_{y}:[-s, 0] \rightarrow X$ por $\widetilde{\sigma}_{y}(r)=\tilde{\pi}(w, s+r)$. Como $s$ é arbitrário, podemos obter uma semisolução negativa impulsiva definida no intervalo $(-\infty, 0]$.

\section{Caso 2: $w \in M$.}

Como $M$ satisfaz a condição STC, existe um STC-tubo $F(L,[0,2 \lambda]), \lambda<\gamma$, através de $w$ dado pela seção $S \subset M$ tal que $S=M \cap F(L,[0,2 \lambda])$. Como o tubo é uma vizinhança de $w$, existe $\xi>0$ tal que $B(w, \xi) \subset F(L,[0,2 \lambda])$. Denotemos $A_{1}$ e $A_{2}$ por

$$
A_{1}=F(L,(\lambda, 2 \lambda]) \cap B(w, \xi) \quad \text { e } \quad A_{2}=F(L,[0, \lambda]) \cap B(w, \xi) .
$$

Afirmamos que $\left\{z_{n}\right\}_{n \geq 1}$ não admite subsequência em $A_{2}$. Suponhamos, por contradição, que exista uma subsequência (denotada, também, por $\left.\left\{z_{n}\right\}_{n \geq 1}\right)$ tal que $z_{n}=\widetilde{\sigma}_{x}\left(t_{n}-s\right) \in A_{2}$ para todo $n=1,2,3, \ldots$ Então pela condição de tubo, existe $\alpha_{n} \in(0, \lambda)$ tal que $z_{n} \in F\left(L, \alpha_{n}\right), n=1,2, \ldots$. Consequentemente, $F\left(z_{n}, \lambda-\alpha_{n}\right) \subset S$ para cada $n=1,2, \ldots$. Como $\pi(M,[0, \gamma]) \cap N=\emptyset$, então existe $a_{n} \in M$ tal que $\widetilde{\sigma}_{x}\left(t_{n}-s+\alpha_{n}-\lambda\right)=a_{n}$, o que é uma contradição, pois $\widetilde{\sigma}_{x}$ é definido no intervalo $(-\infty, 0]$. Portanto, podemos assumir que $z_{n}=\widetilde{\sigma}_{x}\left(t_{n}-s\right) \in A_{1}$ para todo $n=1,2, \ldots$. Neste caso, $\phi\left(z_{n}\right) \stackrel{n \rightarrow+\infty}{\longrightarrow} 0 \mathrm{e}$

$$
\widetilde{\sigma}_{x}\left(t_{n}-s+\phi\left(z_{n}\right)\right)=\tilde{\pi}\left(\widetilde{\sigma}_{x}\left(t_{n}-s\right), \phi\left(z_{n}\right)\right)=\tilde{\pi}\left(z_{n}, \phi\left(z_{n}\right)\right) \stackrel{n \rightarrow+\infty}{\longrightarrow} I(w),
$$


com $I(w) \notin M$. Observe que $I(w) \in \widetilde{L}_{\sigma}^{-}(x) \backslash M$. Então,

$$
\tilde{\pi}\left(y, \eta_{\ell}\right) \stackrel{n \rightarrow+\infty}{\longleftarrow} \tilde{\sigma}_{x}\left(t_{n}+\eta_{\ell}\right)=\tilde{\pi}\left(\widetilde{\sigma}_{x}\left(t_{n}-s+\phi\left(z_{n}\right)\right), s+\eta_{\ell}-\phi\left(z_{n}\right)\right) \stackrel{n \rightarrow+\infty}{\longrightarrow} \tilde{\pi}\left(I(w), s+\eta_{\ell}\right) .
$$

Assim, $\tilde{\pi}\left(I(w), s+\eta_{\ell}\right)=\tilde{\pi}\left(y, \eta_{\ell}\right)$ para todo $\ell=1,2, \ldots$. Usando, mais uma vez, a continuidade à direita de $\tilde{\pi}_{x}$, temos $\tilde{\pi}(I(w), s)=y$ quando $\eta_{\ell} \stackrel{\ell \rightarrow+\infty}{\longrightarrow} 0$. De forma análoga ao caso anterior, podemos definir $\widetilde{\sigma}_{y}:[-s, 0] \rightarrow X$ por

$$
\widetilde{\sigma}_{y}(r)=\tilde{\pi}(I(w), s+r) .
$$

Como $s$ foi escolhido arbitrário, podemos obter uma semisolução negativa impulsiva definida no intervalo $(-\infty, 0]$. O teorema está provado.

O Teorema 5.3.7 apresenta condições para que o conjunto limite positivo seja negativamente fracamente invariante. A prova é semelhante a demonstração do Teorema 5.3.6.

Teorema 5.3.7 [15, Teorema 4.5] Seja $(X, \pi ; M, I)$ um sistema semidinâmico impulsivo e suponha que exista $\gamma>0$ tal que $\pi(M,[0, \gamma]) \cap N=\emptyset$. Seja $x \in X$ tal que $\overline{\tilde{\pi}^{+}(x)}$ é compacto. Então $\widetilde{L}^{+}(x) \backslash M$ é negativamente fracamente invariante.

Demonstração: Seja $y \in \widetilde{L}^{+}(x) \backslash M$. Então existe uma sequência $\left\{t_{n}\right\}_{n \geq 1} \subset \mathbb{R}_{+}$tal que $t_{n} \stackrel{n \rightarrow+\infty}{\longrightarrow}+\infty$ e $\tilde{\pi}\left(x, t_{n}\right) \stackrel{n \rightarrow+\infty}{\longrightarrow} y$. Seja $s \geq 0$ fixo e arbitrário. Podemos assumir que $t_{n}-s>0$ para todo $n$. Defina $z_{n}=\tilde{\pi}\left(x, t_{n}-s\right), n=1,2, \ldots$. Pela compacidade de $\overline{\tilde{\pi}^{+}(x)}$, podemos supor, sem perda de generalidade, que $z_{n} \stackrel{n \rightarrow+\infty}{\longrightarrow} w \in \widetilde{L}^{+}(x)$. Usando o Lema 1.4.6, obtemos

$$
\tilde{\pi}\left(x, t_{n}+\eta\right) \stackrel{n \rightarrow+\infty}{\longrightarrow} \tilde{\pi}(y, \eta)
$$

para todo $\eta \in[0, \phi(y))$. Seja $\left\{\eta_{\ell}\right\}_{\ell \geq 1} \subset \mathbb{R}_{+}$uma sequência não crescente de números positivos tal que $\eta_{\ell} \stackrel{\ell \rightarrow+\infty}{\longrightarrow} 0$. Podemos assumir que existem números $k, p \in \mathbb{N}$ tais que

$$
\sum_{j=-1}^{k-1} \phi\left(w_{j}^{+}\right)<s+\eta_{\ell}<\sum_{j=-1}^{k} \phi\left(w_{j}^{+}\right) \quad \text { e } \sum_{j=-1}^{p-1} \phi\left(I(w)_{j}^{+}\right)<s+\eta_{\ell}<\sum_{j=-1}^{p} \phi\left(I(w)_{j}^{+}\right)
$$

para todo $\ell=1,2, \ldots$, onde $\phi\left(w_{-1}^{+}\right)=\phi\left(I(w)_{-1}^{+}\right)=0$. Vamos considerar os seguintes casos:

Caso 1: $w \notin M$.

Neste caso, usando o Lema 1.4.6 e (5.10) obtemos 


$$
\tilde{\pi}\left(w, s+\eta_{\ell}\right) \stackrel{n \rightarrow+\infty}{\longleftarrow} \tilde{\pi}\left(z_{n}, s+\eta_{\ell}\right)=\tilde{\pi}\left(x, t_{n}+\eta_{\ell}\right) \stackrel{n \rightarrow+\infty}{\longrightarrow} \tilde{\pi}\left(y, \eta_{\ell}\right) .
$$

Assim, $\tilde{\pi}\left(w, s+\eta_{\ell}\right)=\tilde{\pi}\left(y, \eta_{\ell}\right)$ para todo $\ell=1,2, \ldots$. Como $\tilde{\pi}_{x}$ é contínua à direita, obtemos $\tilde{\pi}(w, s)=y$ quando $\eta_{\ell} \stackrel{\ell \rightarrow+\infty}{\longrightarrow} 0$.

Por outro lado, como $\widetilde{L}^{+}(x) \backslash M$ é positivamente $\tilde{\pi}$-invariante, temos que $\widetilde{\pi}^{+}(w) \subset \widetilde{L}^{+}(x)$. Então $\tilde{\pi}(w,[0, s]) \subset \widetilde{L}^{+}(x)$ e $\tilde{\pi}(w, s)=y$. Logo, podemos definir $\widetilde{\sigma}_{y}:[-s, 0] \rightarrow X$ por

$$
\widetilde{\sigma}_{y}(r)=\tilde{\pi}(w, s+r)
$$

Como $s$ é arbitrário, podemos extender $\widetilde{\sigma}_{y}$ a uma semisolução negativa impulsiva definida no intervalo $(-\infty, 0]$.

Caso 2: $w \in M$. Como $M$ satisfaz a condição STC, existe um STC-tubo $F(L,[0,2 \lambda]), \lambda<\gamma$, através de $w$ dado pela seção $S \subset M$ tal que $S=M \cap F(L,[0,2 \lambda])$. Como o tubo é uma vizinhança de $w$, existe $\xi>0$ tal que $B(w, \xi) \subset F(L,[0,2 \lambda])$. Denotemos $A_{1}$ e $A_{2}$ por

$$
A_{1}=F(L,(\lambda, 2 \lambda]) \cap B(w, \xi) \quad \text { e } \quad A_{2}=F(L,[0, \lambda]) \cap B(w, \xi) .
$$

Afirmamos que $\left\{z_{n}\right\}_{n \geq 1}$ não admite subsequência em $A_{2}$. Suponhamos, por contradição, que exista uma subsequência (denotada por $\left\{z_{n}\right\}_{n \geq 1}$ ) tal que $z_{n}=\tilde{\pi}\left(x, t_{n}-s\right) \in A_{2}$ para todo $n=$ $1,2,3, \ldots$ Então pela condição de tubo, existe $\alpha_{n} \in(0, \lambda)$ tal que $z_{n} \in F\left(L, \alpha_{n}\right), n=1,2, \ldots$. Consequentemente, $F\left(z_{n}, \lambda-\alpha_{n}\right) \subset S$ para cada $n=1,2, \ldots$. Como não existe $b_{n} \in M$ tal que $\tilde{\pi}\left(b_{n}, \lambda-\alpha_{n}\right)=z_{n}$, segue que existe $a_{n} \in M$ tal que $I\left(a_{n}\right)=z_{n}$, o que é uma contradição, pois $\pi(M,[0, \gamma]) \cap I(M)=\emptyset$. Portanto, podemos assumir que $z_{n} \in A_{1}$ para todo $n=1,2, \ldots$. Neste caso, $\phi\left(z_{n}\right) \stackrel{n \rightarrow+\infty}{\longrightarrow} 0 \mathrm{e}$

$$
\tilde{\pi}\left(x, t_{n}-s+\phi\left(z_{n}\right)\right)=\tilde{\pi}\left(z_{n}, \phi\left(z_{n}\right)\right) \stackrel{n \rightarrow+\infty}{\longrightarrow} I(w),
$$

com $I(w) \notin M$. Observe que $I(w) \in \widetilde{L}^{+}(x) \backslash M$. Então,

$$
\tilde{\pi}\left(y, \eta_{\ell}\right) \stackrel{n \rightarrow+\infty}{\longleftarrow} \tilde{\pi}\left(x, t_{n}+\phi\left(z_{n}\right)+\eta_{\ell}\right)=\tilde{\pi}\left(z_{n}, \phi\left(z_{n}\right)+s+\eta_{\ell}\right) \stackrel{n \rightarrow+\infty}{\longrightarrow} \tilde{\pi}\left(I(w), s+\eta_{\ell}\right) .
$$


Assim, $\tilde{\pi}\left(I(w), s+\eta_{\ell}\right)=\tilde{\pi}\left(y, \eta_{\ell}\right)$ para todo $\ell=1,2, \ldots$. Usando, mais uma vez, a continuidade à direita de $\tilde{\pi}_{x}$, temos $\tilde{\pi}(I(w), s)=y$ quando $\eta_{\ell} \stackrel{\ell \rightarrow+\infty}{\longrightarrow} 0$. De forma análoga ao caso anterior, podemos definir $\widetilde{\sigma}_{y}:[-s, 0] \rightarrow X$ por

$$
\widetilde{\sigma}_{y}(r)=\tilde{\pi}(I(w), s+r) .
$$

Como $s$ foi escolhido arbitrário, obtemos uma semisolução negativa impulsiva definida no intervalo $(-\infty, 0]$. O teorema está provado.

O resultado seguinte apresenta uma caracterização para o fecho de uma semisolução negativa.

Lema 5.3.8 [15, Lema 4.4] Sejam $(X, \pi ; M, I)$ um sistema semidinâmico impulsivo, $x \in X \backslash M e$ $\widetilde{\sigma}_{x}$ uma semisolução negativa impulsiva através de $x$ definida no intervalo $(-\infty, 0]$. Então

a) $\overline{\widetilde{\sigma}_{x}((-\infty, 0])}=\widetilde{\sigma}_{x}((-\infty, 0]) \cup\left\{a_{n}^{-}\right\}_{1 \leq n \leq k} \cup \widetilde{L}_{\sigma}^{-}(x)$ se $x \in J_{k}$ para algum $k \in \mathbb{N}$.

b) $\overline{\widetilde{\sigma}_{x}((-\infty, 0])}=\widetilde{\sigma}_{x}((-\infty, 0]) \cup\left\{a_{n}^{-}\right\}_{n \geq 1} \cup \widetilde{L}_{\sigma}^{-}(x)$ se $x \in J_{\infty}$.

Demonstração: Vamos mostrar o item $b$ ). Vamos considerar $x \notin N$, pois o caso $x \in N$ é análogo. Seja $y \in \overline{\widetilde{\sigma}_{x}((-\infty, 0])}$. Então existe uma sequência $\left\{t_{n}\right\}_{n \geq 1} \subset \mathbb{R}_{-}$tal que $\widetilde{\sigma}_{x}\left(t_{n}\right) \stackrel{n \rightarrow+\infty}{\longrightarrow} y$.

Se $t_{n} \stackrel{n \rightarrow+\infty}{\longrightarrow}-\infty$, então $y \in \widetilde{L}_{\sigma}^{-}(x)$.

Por outro lado, se existe uma subsequência $\left\{t_{n_{k}}\right\}_{k \geq 1}$ tal que $t_{n_{k}} \stackrel{n \rightarrow+\infty}{\longrightarrow} t_{0}$, devemos considerar os seguintes casos.

Caso 1: $t_{0}$ não é um ponto impulsivo, isto é, $T_{0}<t_{0} \leq 0$ ou $T_{j}<t_{0}<T_{j-1}$ para algum $j \in$ $\{1,2, \ldots\}$.

Se $T_{0}<t_{0} \leq 0$, então $t_{n_{k}} \in\left(T_{0}, 0\right]$ para $n_{k}$ suficientemente grande, logo

$$
\widetilde{\sigma}_{x}\left(t_{n_{k}}\right)=\sigma_{x}\left(t_{n_{k}}\right) \stackrel{k \rightarrow+\infty}{\longrightarrow} \sigma_{x}\left(t_{0}\right)=\widetilde{\sigma}_{x}\left(t_{0}\right)
$$

Agora, se $T_{j}<t_{0}<T_{j-1}$, então $t_{n_{k}} \in\left(T_{j}, T_{j-1}\right)$ para $n_{k}$ suficientemente grande. Assim,

$$
\tilde{\sigma}_{x}\left(t_{n_{k}}\right)=\sigma_{a_{j}^{-}}\left(t_{n_{k}}-T_{j-1}\right) \stackrel{k \rightarrow+\infty}{\longrightarrow} \sigma_{a_{j}^{-}}\left(t_{0}-T_{j-1}\right)=\widetilde{\sigma}_{x}\left(t_{0}\right)
$$


Portanto, $y=\widetilde{\sigma}_{x}\left(t_{0}\right) \in \widetilde{\sigma}_{x}((-\infty, 0])$.

Caso 2: $t_{0}$ é um ponto de impulso, isto é, $t_{0}=T_{j}$ para algum $j \in \mathbb{N}$.

Se $\left\{t_{n_{k}}\right\}_{k \geq 1}$ possui uma subsequência, a qual denotamos ainda por $\left\{t_{n_{k}}\right\}_{k \geq 1}$, tal que $t_{n_{k}} \geq t_{0}$ para todo $k=1,2, \ldots$, então

$$
\widetilde{\sigma}_{x}\left(t_{n_{k}}\right)=\sigma_{a_{j}^{-}}\left(t_{n_{k}}-T_{j-1}\right) \stackrel{k \rightarrow+\infty}{\longrightarrow} \sigma_{a_{j}^{-}}\left(t_{0}-T_{j-1}\right)=\widetilde{\sigma}_{x}\left(t_{0}\right)
$$

Assim, $y=\widetilde{\sigma}_{x}\left(t_{0}\right) \in \widetilde{\sigma}_{x}((-\infty, 0])$.

Agora, se $\left\{t_{n_{k}}\right\}_{k \geq 1}$ possui uma subsequência, a qual denotamos ainda por $\left\{t_{n_{k}}\right\}_{k \geq 1}$, tal que $t_{n_{k}}<t_{0}$ para todo $k=1,2, \ldots$, então

$$
\widetilde{\sigma}_{x}\left(t_{n_{k}}\right)=\sigma_{a_{j+1}^{-}}\left(t_{n_{k}}-T_{j}\right) \stackrel{k \rightarrow+\infty}{\longrightarrow} \sigma_{a_{j+1}^{-}}(0)=a_{j+1}^{-}
$$

Portanto, $y=a_{j+1}^{-} \in\left\{a_{n}^{-}\right\}_{n \geq 1}$ e o resultado está provado.

Teorema 5.3.9 [15, Teorema 4.6] Seja $(X, \pi ; M, I)$ um sistema semidinâmico impulsivo. Suponha $x \in X, y \in X \backslash M$ e $\widetilde{\sigma}_{y}$ uma semisolução negativa impulsiva através de y definida no intervalo $(-\infty, 0]$. Se $x \in \widetilde{L}_{\sigma}^{-}(y)$ então $y \in \widetilde{J}^{+}(x)$.

Demonstração: Como $x \in \widetilde{L}_{\sigma}^{-}(y)$, então existe uma sequência $\left\{t_{n}\right\}_{n \geq 1} \subset \mathbb{R}_{-}$tal que $t_{n} \stackrel{n \rightarrow+\infty}{\longrightarrow}-\infty$ e $\tilde{\sigma}_{y}\left(t_{n}\right) \stackrel{n \rightarrow+\infty}{\longrightarrow} x$. Observe que $-t_{n}>0$ para todo $n \geq 1$ e $\tilde{\pi}\left(\widetilde{\sigma}_{y}\left(t_{n}\right),-t_{n}\right)=\widetilde{\sigma}_{y}(0)=y$, portanto $y \in \widetilde{J}^{+}(x)$.

Apresentamos, na sequência, o conceito do primeiro prolongamento do conjunto limite negativo de um ponto no contexto dos sistemas impulsivos.

Definição 5.3.10 $O$ primeiro prolongamento do conjunto limite negativo de $x \in X$ é definido pelo seguinte conjunto:

$$
\widetilde{j}^{-}(x)=\left\{y \in X: \text { existem sequências }\left\{x_{n}\right\}_{n \geq 1} \subset X \backslash M \text { e }\left\{t_{n}\right\}_{n \geq 1} \subset \mathbb{R}_{-}\right.
$$
tais que $x_{n} \stackrel{n \rightarrow+\infty}{\longrightarrow} x, t_{n} \stackrel{n \rightarrow+\infty}{\longrightarrow}-\infty$ e para cada $x_{n}$ existe uma semisolução 
negativa impulsiva $\widetilde{\sigma}_{n}$ através de $x_{n}$ definida no intervalo $(-\infty, 0]$ tal que $\left.\widetilde{\sigma}_{n}\left(t_{n}\right) \stackrel{n \rightarrow+\infty}{\longrightarrow} y\right\}$.

Teorema 5.3.11 [15, Teorema 4.7] Seja $(X, \pi ; M, I)$ um sistema semidinâmico impulsivo. Para qualquer $x \in X$ o conjunto $\widetilde{j}^{-}(x)$ é fechado e $\widetilde{j}^{-}(x) \backslash M$ é positivamente $\tilde{\pi}$-invariante.

Demonstração: Mostremos que $\widetilde{j}^{-}(x)$ é fechado, $x \in X$. Seja $\left\{y_{n}\right\}_{n \geq 1}$ uma sequência em $\widetilde{j}^{-}(x)$ tal que

$$
y_{n} \stackrel{n \rightarrow+\infty}{\longrightarrow} y .
$$

Para cada $n \in \mathbb{N}^{*}$, existem uma sequência $\left\{x_{k}^{n}\right\}_{k \geq 1} \subset X \backslash M$ e $\left\{t_{k}^{n}\right\}_{k \geq 1} \subset \mathbb{R}_{-}$tais que

$$
x_{k}^{n} \stackrel{k \rightarrow+\infty}{\longrightarrow} x \text { e } t_{k}^{n} \stackrel{k \rightarrow+\infty}{\longrightarrow}-\infty
$$

além disso, para cada $x_{k}^{n}$ existe uma semisolução negativa impulsiva $\widetilde{\sigma}_{x_{k}^{n}}$ através de $x_{k}^{n}$ definida em $(-\infty, 0] \operatorname{com}$

$$
\widetilde{\sigma}_{x_{k}^{n}}\left(t_{k}^{n}\right) \stackrel{k \rightarrow+\infty}{\longrightarrow} y_{n}
$$

Usando (5.12) temos que, existe $k_{n} \geq n$ tal que

$$
t_{k_{n}}^{n}<-n \text { e } x_{k_{n}}^{n} \stackrel{n \rightarrow+\infty}{\longrightarrow} x
$$

Por outro lado, de (5.11) e (5.13) obtemos

$$
d\left(\widetilde{\sigma}_{k_{k_{n}}^{n}}\left(t_{k_{n}}^{n}\right), y\right) \leq d\left(\widetilde{\sigma}_{x_{k_{n}}^{n}}\left(t_{k_{n}}^{n}\right), y_{n}\right)+d\left(y_{n}, y\right) \stackrel{n \rightarrow+\infty}{\longrightarrow} 0
$$

isto implica que $\widetilde{\sigma}_{x_{k_{n}}^{n}}\left(t_{k_{n}}^{n}\right) \stackrel{n \rightarrow+\infty}{\longrightarrow} y$. Assim, $y \in \widetilde{j}^{-}(x)$ e $\widetilde{j}^{-}(x)$ é fechado.

Provemos, agora, que $\widetilde{j}^{-}(x) \backslash M$ é um conjunto positivamente $\tilde{\pi}$-invariante. Sejam $y \in \widetilde{j}^{-}(x) \backslash M$ e $t \geq 0$ arbitrários. Então existem sequências $\left\{x_{n}\right\}_{n \geq 1} \subset X \backslash M$ e $\left\{t_{n}\right\}_{n \geq 1} \subset \mathbb{R}_{-}$tais que $x_{n} \stackrel{n \rightarrow+\infty}{\longrightarrow} x, t_{n} \stackrel{n \rightarrow+\infty}{\longrightarrow}-\infty$ e para cada $x_{n}$ existe uma semisolução negativa impulsiva $\widetilde{\sigma}_{n}$ através de $x_{n}$ definida em $(-\infty, 0]$ tal que $\widetilde{\sigma}_{n}\left(t_{n}\right) \stackrel{n \rightarrow+\infty}{\longrightarrow} y$. Como $y \notin M$, segue do Lema 1.4.5 que existe uma sequência $\left\{\varepsilon_{n}\right\}_{n \geq 1} \subset \mathbb{R}$ tal que $\varepsilon_{n} \stackrel{n \rightarrow+\infty}{\longrightarrow} 0 \mathrm{e}$

$$
\widetilde{\sigma}_{n}\left(t_{n}+t+\varepsilon_{n}\right)=\tilde{\pi}\left(\widetilde{\sigma}_{n}\left(t_{n}\right), t+\varepsilon_{n}\right) \stackrel{n \rightarrow+\infty}{\longrightarrow} \widetilde{\pi}(y, t)
$$


Como $t_{n}+t+\varepsilon_{n} \stackrel{n \rightarrow+\infty}{\longrightarrow}-\infty$, obtemos $\tilde{\pi}(y, t) \in \widetilde{j}^{-}(x)$

O Teorema 5.3.12 estabelece uma relação entre o prolongamento do conjunto limite positivo e o primeiro prolongamento do conjunto limite negativo.

Teorema 5.3.12 [15, Teorema 4.8] Sejam $(X, \pi ; M, I)$ um sistema semidinâmico impulsivo e $x, y \in$ $X$. Se $x \in \widetilde{j}^{-}(y)$ então $y \in \widetilde{J}^{+}(x)$.

Demonstração: Seja $x \in \widetilde{j}^{-}(y)$. Então existem sequências $\left\{y_{n}\right\}_{n \geq 1} \subset X \backslash M$ e $\left\{t_{n}\right\}_{n \geq 1} \subset \mathbb{R}_{-}$tais que $y_{n} \stackrel{n \rightarrow+\infty}{\longrightarrow} y, t_{n} \stackrel{n \rightarrow+\infty}{\longrightarrow}-\infty$ e para cada $y_{n}$ existe uma semisolução negativa impulsiva $\widetilde{\sigma}_{n}$ através de $y_{n}$ definida no intervalo $(-\infty, 0]$ tal que $\widetilde{\sigma}_{n}\left(t_{n}\right) \stackrel{n \rightarrow+\infty}{\longrightarrow} x$. Para cada $n \in \mathbb{N}^{*}$, defina $x_{n}=\widetilde{\sigma}_{n}\left(t_{n}\right) \mathrm{e}$ $\tau_{n}=-t_{n}$. Então $x_{n} \stackrel{n \rightarrow+\infty}{\longrightarrow} x, \tau_{n} \stackrel{n \rightarrow+\infty}{\longrightarrow}+\infty \mathrm{e}$

$$
\tilde{\pi}\left(x_{n}, \tau_{n}\right)=\tilde{\pi}\left(\widetilde{\sigma}_{n}\left(t_{n}\right),-t_{n}\right)=\widetilde{\sigma}_{n}(0)=y_{n} \stackrel{n \rightarrow+\infty}{\longrightarrow} y .
$$

Isto implica que $y \in \widetilde{J}^{+}(x)$.

\subsection{Conjuntos fracamente minimais}

No Capítulo 2, apresentamos o conceito de conjuntos minimais para sistemas semidinâmicos impulsivos. Este conceito foi estabelecido como uma extensão da definição de minimalidade da teoria clássica de sistemas dinâmicos contínuos. Nesta seção, estabelecemos o conceito de conjuntos minimais para sistemas semidinâmicos impulsivos que admitem soluções negativas.

Definição 5.4.1 Um conjunto $A \subset X$ é (fortemente) (fracamente) minimal em $(X, \pi ; M, I)$ se as seguintes condições forem satisfeitas:

i) $A \backslash M \neq \emptyset$;

ii) A é fechado;

iii) $A \backslash M$ é (fortemente)(fracamente) invariante;

iv) A não admite subconjunto próprio satisfazendo $i$ ), ii) e iii). 
É claro que $A$ não é necessariamente fortemente minimal se ele for fracamente minimal. No entanto, temos o seguinte resultado.

Teorema 5.4.2 [15, Teorema 4.9] Seja $(X, \pi ; M, I)$ um sistema semidinâmico impulsivo. Se $A \subset X$ é fracamente minimal e negativamente fortemente invariante, então A é fortemente minimal.

Definição 5.4.3 Um conjunto $U \subset X$ é chamado trivial se existe $x \in U \backslash M$ tal que $\tilde{\pi}_{\sigma}(x)=U \backslash M$.

O Teorema 5.4.4 lida com algumas propriedades sobre conjuntos fracamente minimais e negativamente fortemente invariantes.

Teorema 5.4.4 [15, Teorema 4.10] Seja $(X, \pi ; M, I)$ um sistema semidinâmico impulsivo tal que $\pi(M,[0, \gamma]) \cap N=\emptyset$ para algum $\gamma>0$. Suponha que $A \subset X$ é um conjunto não trivial, fracamente minimal e negativamente fortemente invariante. Se a trajetória através de $x \in X \backslash M$ está contida em A então nenhuma das seguintes propriedades estão satisfeitas:

a) $\widetilde{L}^{+}(x)=\emptyset$ e existe uma semisolução negativa impulsiva $\widetilde{\sigma}_{x}$ através de $x$ definida no intervalo $(-\infty, 0]$ tal que $\widetilde{L}_{\sigma}^{-}(x)=\emptyset$;

b) $\widetilde{L}^{+}(x) \backslash M \neq \emptyset, \widetilde{L}^{+}(x) \cap \widetilde{\pi}^{+}(x)=\emptyset e \overline{\widetilde{\pi}^{+}(x)}$ é compacto;

c) existe uma semisolução negativa impulsiva $\widetilde{\sigma}_{x}$ através de $x$ definida no intervalo $(-\infty, 0]$ tal que $\overline{\widetilde{\sigma}_{x}((-\infty, 0])}$ é compacto, $\widetilde{L}_{\sigma}^{-}(x) \backslash M \neq \emptyset e \widetilde{L}_{\sigma}^{-}(x) \cap \widetilde{\sigma}_{x}((-\infty, 0])=\emptyset$.

Demonstração: $a$ ) Suponhamos que $\widetilde{L}^{+}(x)=\emptyset$ e $\widetilde{L}_{\sigma}^{-}(x)=\emptyset$ para alguma semisolução negativa impulsiva $\widetilde{\sigma}_{x}$ através de $x$ definida no intervalo $(-\infty, 0]$. Pelos Lemas 1.4 .11 e 5.3.8, podemos considerar o caso em que

$$
\overline{\tilde{\pi}_{\sigma}(x)}=\tilde{\pi}^{+}(x) \cup\left\{x_{k}\right\}_{k \geq 1} \cup \widetilde{\sigma}_{x}((-\infty, 0]) \cup\left\{a_{j}^{-}\right\}_{j \in \mathbb{N}} .
$$

Assim,

$$
\overline{\tilde{\pi}_{\sigma}(x)} \backslash M=\tilde{\pi}^{+}(x) \cup \widetilde{\sigma}_{x}((-\infty, 0])=\tilde{\pi}_{\sigma}(x) .
$$


É claro que $\overline{\widetilde{\pi}_{\sigma}(x)}$ é fechado e $\overline{\widetilde{\pi}_{\sigma}(x)} \backslash M \neq \emptyset$. Do Lema 5.3.2, segue que $\overline{\widetilde{\pi}_{\sigma}(x)} \backslash M$ é fracamente invariante. $\operatorname{Logo} A=\bar{\pi}_{\sigma}(x)$, pois $A$ é fracamente minimal. Portanto $A \backslash M=\tilde{\pi}_{\sigma}(x)$ e isto contradiz a não trivialidade do conjunto $A$.

b) Suponhamos que $\widetilde{L}^{+}(x) \backslash M \neq \emptyset, \widetilde{L}^{+}(x) \cap \widetilde{\pi}^{+}(x)=\emptyset$ e $\overline{\widetilde{\pi}^{+}(x)}$ é compacto. Então $\widetilde{L}^{+}(x) \subset$ $A$, pois $\tilde{\pi}_{\sigma}(x) \subset A$ e $A$ é fechado. Como $x \notin \widetilde{L}^{+}(x)$, segue que $\widetilde{L}^{+}(x) \neq A$. Mas isso é uma contradição, pois $\widetilde{L}^{+}(x) \backslash M$ é negativamente fracamente invariante (Teorema 5.3.7) e positivamente $\tilde{\pi}$-invariante, $\widetilde{L}^{+}(x)$ é fechado e $A$ é fracamente minimal.

c) Suponhamos que exista uma semisolução negativa impulsiva $\widetilde{\sigma}_{x}$ através de $x$ definida no intervalo $(-\infty, 0]$ tal que $\widetilde{L}_{\sigma}^{-}(x) \backslash M \neq \emptyset, \widetilde{L}_{\sigma}^{-}(x) \cap \widetilde{\sigma}_{x}((-\infty, 0])=\emptyset$ e $\overline{\widetilde{\sigma}_{x}((-\infty, 0])}$ compacto. Note que $\overline{\widetilde{\sigma}_{x}((-\infty, 0])} \subset A$, pois $A$ é negativamente fortemente invariante e fechado. Então,

$$
\widetilde{L}_{\sigma}^{-}(x) \subset A \quad \text { e } \quad \widetilde{L}_{\sigma}^{-}(x) \neq A
$$

Pelo Teorema 5.3.6 temos que $\widetilde{L}_{\sigma}^{-}(x) \backslash M$ é negativamente fracamente invariante. Além disso, pelo Teorema 5.3.5, $\widetilde{L}_{\sigma}^{-}(x) \backslash M$ é positivamente $\widetilde{\pi}$-invariante. Pela minimalidade de $A$ temos $A=\widetilde{L}_{\sigma}^{-}(x)$ e isto é uma contradição. 


\section{Referências Bibliográficas}

[1] A. Bistroń, On minimal and invariant sets in semidynamical systems, Universitatis Iagellonicae Acta Mathematicae, Fasciculus XLI, (2003), 189-204.

[2] N. P. Bhatia e O. Hajek, Local semi-dynamical systems, Lecture Notes in Mathematics 90, Springer-Verlag, 1970.

[3] N. P. Bhatia e G. P. Szegö, Stability theory of dynamical systems, Grundlehren Math. Wiss., Band 161, Springer-Verlag, New York, 1970; reprint of the 1970 original in: Classics Math., Springer-Verlag, Berlin, 2002.

[4] E. M. Bonotto, Sistemas Semidinâmicos Impulsivos. Dissertação de Mestrado. Universidade de São Paulo, 2005.

[5] E. M. Bonotto e M. Federson, Topological conjugation and asymptotic stability in impulsive semidynamical systems. J. Math. Anal. Appl. 326, (2007), 869-881.

[6] E. M. Bonotto, Flows of characteristic $0^{+}$in impulsive semidynamical systems, J. Math. Anal. Appl., 332 (1), (2007), 81-96.

[7] E. M. Bonotto e M. Federson, Limit sets and the Poincaré-Bendixson Theorem in semidynamical impulsive systems. J. Diff. Equations. 244 (2008), 2334-2349.

[8] E. M. Bonotto, LaSalle's Theorems in impulsive semidynamical systems. Nonlinear Analysis: Theory, Methods \& Applications. 71 (5-6), (2009), 2291-2297.

[9] E. M. Bonotto e M. Federson, Poisson stability for impulsive semidynamical systems. Nonlinear Analysis: Theory, Methods \& Applications, 71 (12), (2009), 6148-6156. 
[10] E. M. Bonotto e N. G. Grulha Jr., Lyapunov stability of closed sets in impulsive semidynamical systems. Eletronic Journal of Differential Equations, 78, (2010), 1-18.

[11] E. M. Bonotto e D. P. Demuner, Autonomous dissipative semidynamical systems with impulses. Topological Methods in Nonlinear Analysis, 41 (1), (2013), 1-38.

[12] E. M. Bonotto e J. C. Ferreira, Uniform attractors in impulsive semidynamical systems. Journal of Dynamical and Control Systems. (2013). DOI 10.1007/s13348-012-0078-8.

[13] E. M. Bonotto e M. Z. Jimenez, On impulsive semidynamical systems: minimal, recurrent and almost periodic motions. Topological Methods in Nonlinear Analysis. Accepted.

[14] E. M. Bonotto e M. Z. Jimenez, Weak almost periodic motions, minimality and stability in impulsive semidynamical systems. Journal of Differential Equations. Accepted.

[15] S. M. Afonso, E. M. Bonotto e M. Z. Jimenez, Negative trajectories in impulsive semidynamical systems. Pré-print.

[16] K. Ciesielski, Sections in semidynamical systems, Bull. Polish Acad. Sci. Math., 40, (1992), 297-307.

[17] K. Ciesielski, On semicontinuity in impulsive dynamical systems, Bull. Polish Acad. Sci. Math., 52, (2004), 71-80.

[18] K. Ciesielski, On stability in impulsive dynamical systems, Bull. Polish Acad. Sci. Math., 52, (2004), 81-91.

[19] C. Ding, Lyapunov quasi-stable trajectories. Fundamenta Mathematicae. 220 (2013), 139154.

[20] S. K. Kaul, On impulsive semidynamical systems, J. Math. Anal. Appl., 150, (1990), 120-128.

[21] S. K. Kaul, On impulsive semidynamical systems II, Recursive properties. Nonlinear Anal., 16, (1991), 635-645.

[22] S. K. Kaul, On impulsive semidynamical systems III, Lyapunov stability. Recent Trends in Differential Equations, 335-345, World Sci. Ser. Appl. Anal., 1. Publishing, River Edge, NJ, 1992. 
[23] S. K. Kaul, Stability and asymptotic stability in impulsive semidynamical systems. J. Appl. Math. Stochastic Anal., 7(4), (1994), 509-523.

[24] V. Lakshmikanthan, D. D. Bainov e P. S. Simeonov, Theory of Impulsive Differential Equations, Modern Applied Math., 6, World Scientific, 1989.

[25] J. H. Mai e W. H. Sun, Almost periodic points and minimal sets in $\omega$-regular spaces, Topology and its Applications, 154 (2007), 2873-2879.

[26] R. C. McCann, Negative escape time in semidynamical systems, Funkc. Ekvac., 20 (1977), 39-47.

[27] V. V. Nemytskii e V. V. Stepanov, Qualitative theory of differential equations, Princeton University Press, 1960. 